\title{
IMPACT OF ENVIRONMENTAL STRESSORS ON THE METABOLIC FUNCTIONING OF A TEMPERATE CNIDARIAN-DINOFLAGELLATE SYMBIOSIS
}

\author{
BY \\ OLIVER GRAHAM BONE
}

\begin{abstract}
A thesis
submitted to Victoria University of Wellington

in partial fulfilment of the requirements for the degree of

Master of Science in Marine Biology
\end{abstract}

Victoria University of Wellington

2016

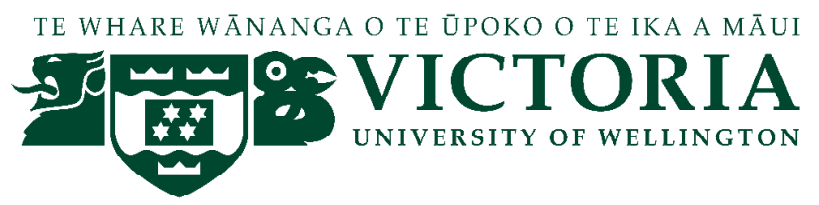




\begin{abstract}
Cnidarian-dinoflagellate symbioses occur across a wide latitudinal range, from temperate to tropical locations in both hemispheres. In the tropics, this association provides the foundation for the development of highly diverse coral reef ecosystems. Tropical associations are particularly sensitive to thermal variability, however, leading to dysfunction of the relationship and eventual expulsion of the symbiont, known as 'coral bleaching'. In contrast, temperate associations maintain stable symbiotic relationships in highly fluctuating thermal environments. The reason behind the relative thermal tolerance of temperate associations is still unclear, though the ability to maintain cellular homeostasis through adjustments to metabolic processes is likely a core feature of their resilience.
\end{abstract}

Both a field study and laboratory experiment were conducted to determine the metabolic responses to thermal change of the symbiosis between the temperate anemone Anthopleura aureoradiata and the dinoflagellate Symbiodinium. For the field component, A. aureoradiata were collected from Point Halswell in Wellington Harbour in both summer and winter. For the laboratory experiment, specimens collected at Pautahanui inlet were thermally acclimated in the laboratory, after which temperatures were altered over the course of one week to either $8^{\circ} \mathrm{C}$ (cold) or $28^{\circ} \mathrm{C}$ (hot) and maintained at these temperatures for six weeks. Gas chromatography coupled to mass spectrometry was then employed to determine the identity and relative quantity of a wide range of metabolites involved in primary metabolism including organic acids, fatty acids, amino acids and sugars. Based on these data, pathway activity profiling was used to determine the activity of different metabolic pathways both between seasons and in response to cold and heat treatment.

A wide range of changes to metabolic processes were observed in both host and symbiont. Photosynthetic capacity was reduced in the symbionts at low temperatures and increased at high temperatures. The only organic acid to be significantly impacted was propanedioic acid, which increased in the host in response to cold treatment, potentially related to increased translocation from the symbiont. Altered fatty acid content in both host and symbiont was related to the role of fatty acids as energy sources and storage compounds 
and in cell signalling processes. Changes in fatty acid-associated metabolic pathways were exemplified by arachidonic acid and linoleic acid metabolism. Alterations to free amino acids and amino acid related pathways in both host and symbiont were associated with their role as antioxidants and osmoprotectants and the catabolism of amino acids for the production of energy. In symbionts only, altered amino acid content was associated with the role of amino acids in the production of alkaloids. Changes in a number of sugar derivatives in both host and symbiont were associated with their role as antioxidants and osmoprotectants. Altered sugar metabolism in the symbiont clearly indicated an increase in the production of energy rich sugar molecules and production of cellular energy in summer/hot conditions and a decrease in winter/cold conditions. Notably impacted pathways included the Calvin cycle, glycolysis, the pentose phosphate pathway and oxidative phosphorylation. Patterns of sugar related pathway activity in the host were generally opposite to that observed in the symbiont. Overall, prominent but opposing changes in the host and symbiont were detected in the central carbohydrate and energy metabolic pathways. In general, the activity of these pathways increased in the host in winter/cold conditions and decreased in summer/hot conditions, while in the symbiont the pattern was the opposite.

These findings highlight the role of metabolic processes in enabling the persistence of a temperate cnidarian-dinoflagellate symbiosis in the face of large temperature fluctuations. This work provides a foundation upon which a deeper understanding of metabolic functioning in the cnidarian-dinoflagellate symbiosis can be built and provides a comparative platform for studies of the more thermally sensitive tropical associations. 


\section{ACKNOWLEDGMENTS}

First and foremost I would like to thank my supervisor, Professor Simon Davy, for his unrelenting support and encouragement throughout this process. I would also like to thank Rob Keyzers for his technical support and guidance while using the GC-MS and Ken Ryan for some pertinent technical pointers regarding use of the freeze dryer

I am grateful to Silas Villas-Boas and other members of the metabolomics lab at the University of Auckland who welcomed me into their workspace and provided a great deal of technical support. In particular, Farhana Pinu, who showed me the ropes of the metabolomics 'R' script and Morgan Hann who kindly ran the PAPi script on my metabolite profile data.

The support and encouragement of my lab colleagues was also immeasurable in the completion of this thesis. In particular I am grateful Jen Mathews, Katie Hillyer and Shaun Wilkinson for generously imparting vital technical lab skills and for always making time to answer questions when needed.

Much support was also provided by the technicians from the School of Biological Sciences. In particular, Craig Dooney and Neville Higginson who made my initial experimental set up possible and especially to Jen Howe who gladly shared her knowledge regarding the collection and maintenance of my study species, Anthopleura aureoradiata.

I am also deeply grateful for the financial support provided to me through the Victoria University of Wellington Masters (by thesis) Scholarship, the Freemasons University Scholarship, the JL Stewart Postgraduate Scholarship, the Alison Morton Scholarship in Ecology \& Marine Biology and a Victoria Graduate Award.

Finally, I would like to acknowledge my friends and family for all their love and support throughout this process. 


\section{TABLE OF CONTENTS}

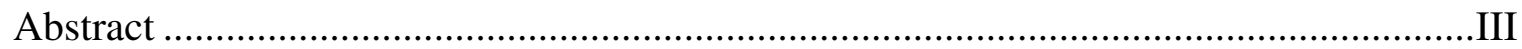

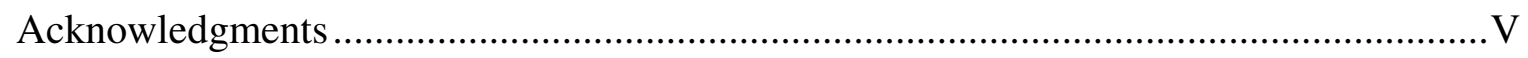

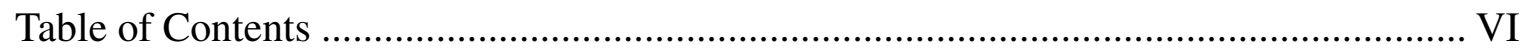

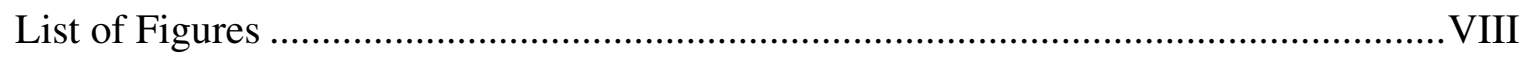

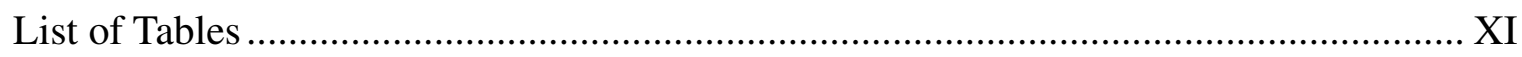

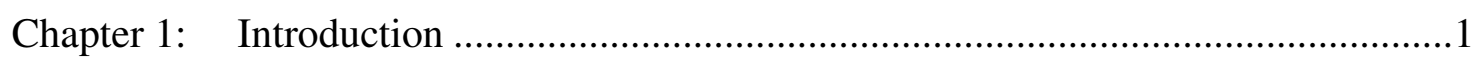

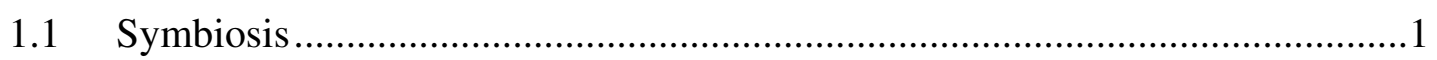

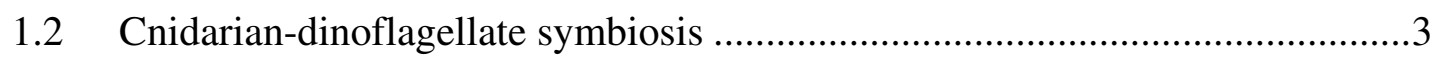

1.3 Temperate cnidarian-dinoflagellate symbioses ...........................................17

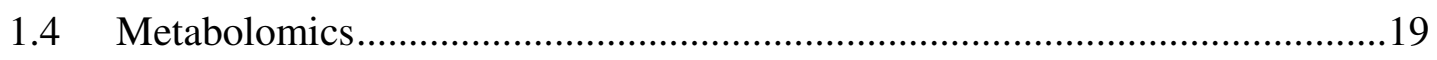

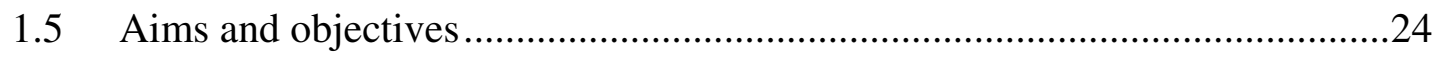

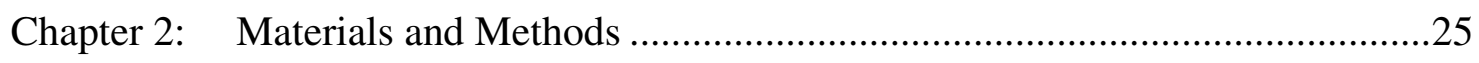

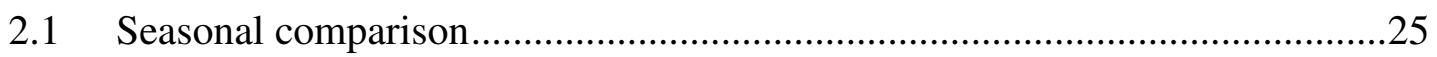

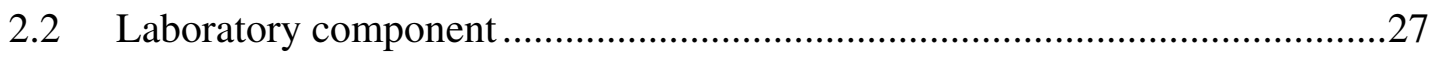

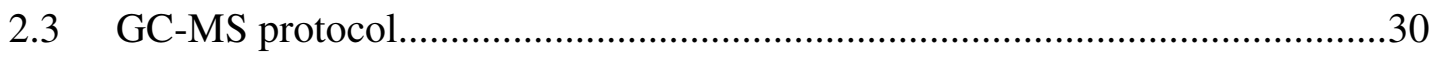

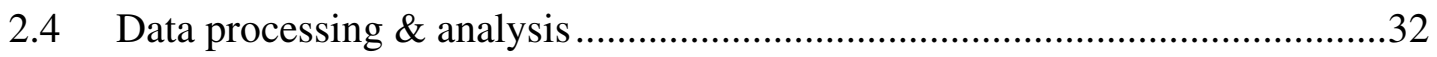

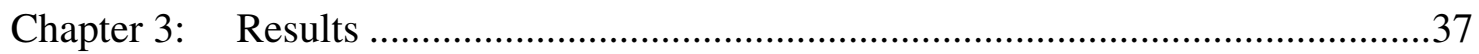

3.1 Exploring seasonal trends in the metabolic functioning of A. aureoradiata and their symbionts

3.2 The impact of thermal change on the metabolic functioning of A. aureoradiata and their symbionts: an experimental approach 


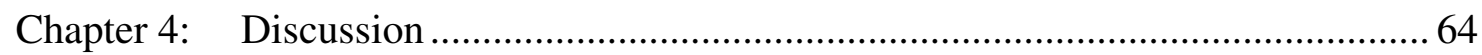

4.1 The impact of environmental variability on symbiotic state ......................... 64

4.2 Metabolic modifications in response to environmental change ......................68 68

4.3 Proposed model of metabolic pathway response to thermal change in the $A$.

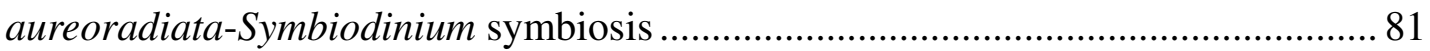

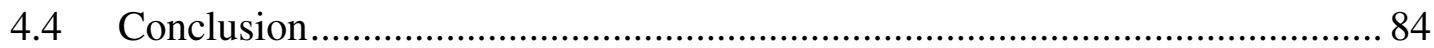

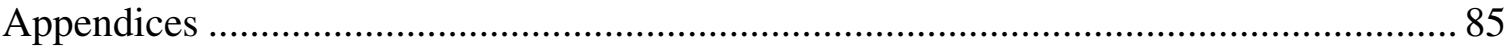

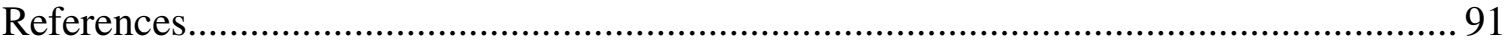




\section{LIST OF FIGURES}

Figure 1.1: A well known and ecologically important example of an obligate, endosymbiotic relationship is the relationship formed between stony corals (Scleractinia) and dinoflagellates (Symbiodinium spp.), here exemplified by the coral Montipora digitata. .3

Figure 1.2: Typical structure of both partners involved in the cnidarian-dinoflagellate symbiosis

Figure 1.3. Nutritional interactions in the cnidarian-dinoflagellate symbiosis. .9

Figure 1.4: Confocal laser-scanning microscopy image of fluorescently-stained mycorrhizal fungus associated with the roots of white clover.

Figure 2.1. Experimental set up 28

Figure 2.2 Common MSTFA silylation reactions (Villas-bôas et al. 2011) Licenced under CC BY 3.0 https://creativecommons.org/licenses/by/3.0/legalcode

Figure 3.1 Weather conditions recorded daily at the Wellington Kelburn automatic weather station (AWS) over a two week period prior to anemone collection in winter 2014 and summer 2015 . 38

Figure 3.2: Total organic carbon (TOC), dissolved reactive phosphorus (DRP), ammonium $\left(\mathrm{NH}_{4}-\mathrm{N}\right)$, nitrate $\left(\mathrm{NO}_{3}-\mathrm{N}\right)$, chlorophyll $a(\mathrm{Chl} a)$ and total chlorophyll (Total Chl) concentrations in surface seawater at the field collection site in winter 2014 and summer 2015.

Figure 3.3: Symbiodinium cell density, and chlorophyll $a$ and $c_{2}$ concentrations per symbiont cell in anemones sampled in winter 2014 and summer 2015. . .40

Figure 3.4: Concentration of individual metabolites per $\mu \mathrm{g}$ dry weight in Symbiodinium $s p$. isolated from A. aureoradiata sampled in either summer or winter.

Figure 3.5: PCA scores plot with shaded $95 \%$ confidence intervals (left) and corresponding loadings plot (right) of symbionts isolated from anemones collected in summer compared to those collected in winter. 
Figure 3.6 Comparison of the activity of key metabolic pathways between seasons in symbionts isolated from A. aureorediata sampled in situ.

Figure 3.7 Concentration of individual metabolites per $\mu$ g dry weight in A. aureoradiata sampled in either summer or winter. 45

Figure 3.8. PCA scores plot with shaded $95 \%$ confidence intervals (left) and corresponding loadings plot (right) of host anemones collected in summer compared to those collected in winter .46

Figure 3.9. Comparison of the activity of key metabolic pathways between seasons in Symbiodinium sp. isolated from A. aureoradiata that were sampled in situ. 47

Figure 3.10: Symbiodinium cell density and chlorophyll $a$ and $c_{2}$ concentrations per symbiont cell in A. aureoradiata under in response to experimental conditions. 49

Figure 3.11. Concentration of individual metabolites per $\mu \mathrm{g}$ dry weight in cold (A) and heat treated (B) symbionts 52

Figure 3.12 PCA scores and corresponding loadings plot for symbionts isolated from $A$. aureoradiata exposed to cold treatment (A) and heat treatment (B) over a 6-week period, relative to controls $\left(15^{\circ} \mathrm{C}\right)$. 54

Figure 3.13 Effect of six weeks of $(\mathrm{A})$ cold $\left(8^{\circ} \mathrm{C}\right)$ and $(\mathrm{B})$ heat $\left(28^{\circ} \mathrm{C}\right)$ treatment on the activity of key symbiont metabolic pathways. 56

Figure 3.14 Concentration of individual metabolites per $\mu \mathrm{g}$ dry weight in A. aureoradiata exposed to cold treatment (A) and heat treatment (B) over a 6-week period, relative to controls $\left(15^{\circ} \mathrm{C}\right)$.

Figure 3.15 PCA scores and corresponding loadings plot for A. aureoradiata exposed to cold treatment (A) and heat treatment (B) over a 6-week period, relative to controls $\left(15^{\circ} \mathrm{C}\right)$. 61

Figure 3.16 Effect of six weeks of $(\mathrm{A})$ cold $\left(8^{\circ} \mathrm{C}\right)$ and $(\mathrm{B})$ heat $\left(28^{\circ} \mathrm{C}\right)$ treatment on the activity of key host metabolic pathways. 63 
Figure 4.1 Metabolic pathways and their response to differing thermal regimes in the

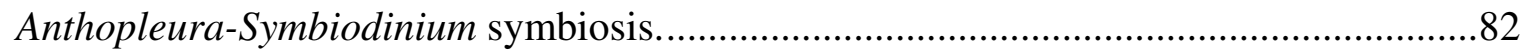




\section{LIST OF TABLES}

Table A 1.1 List of full names matched to names that were concatenated in graphs depicting the results of univariate analysis.

Table A 2.1 Compounds with an unknown function or classification that were identified as being important via fold-change analysis, univariate (t-tests) or multivariate (PCA) analysis in the symbiont. .88

Table A 2.2 Compounds with an unknown function or classification that were identified as being important via fold-change analysis, univariate (t-tests) or multivariate (PCA) analysis in the host 



\section{Chapter 1: Introduction}

\subsection{Symbiosis}

Symbiosis was originally defined by the mycologist Heinrich Anton de Bary as "the living together of unlike organisms" (de Bary 1879), though more recently the concept of symbiosis has been expanded to encompass the notion that the relationship must persist for a significant portion of the lifespan of the organisms involved (Douglas 2010). All symbiotic relationships exist on a continuum ranging from parasitism, where one organism is benefited at the expense of the other, to mutualism, where both organisms gain benefits (Douglas 2010). Parasitism comes in many shapes and forms; but one clear example is malaria. Malaria is caused by the invasion of the human liver and blood cells by a range of protozoan parasites belonging to the genus Plasmodium and has many deleterious effects on human health (Cowman et al. 2012). On the other hand, one well studied mutualism is the association between mycorrhizal fungi and the roots of plants, known as arbuscular mycorrhizal fungi (AMF). In such associations, the mycorrhizal fungi provide nutrients to the host plant (Leigh et al. 2009; Smith \& Smith 2011; Karasawa et al. 2012), and are supplied with photosynthetically-derived carbohydrates in return (Bryla \& Eissenstat 2005). Commensalism, where one organism benefits while the other is neither harmed nor benefits, falls somewhere between the two extremes of parasitism and mutualism (Douglas 2010).

To further understand the symbiotic relationship it should be noted that associations may either be vital to the survival of either partner, known as 'obligate' symbioses, or not vital to the survival of either partner, termed 'facultative' symbioses (Baeza 2007). The aforementioned mycorrhizal-plant association illustrates this concept. In particular, while plants capable of forming AMF associations can function effectively when independent of fungal symbionts, their performance is greatly enhanced as a result of the relationship (Rouphael et al. 2015; Berruti et al. 2016), deeming the plant a facultative symbiont. In contrast, it appears that the fungal partner of AMF associations is completely reliant on the host plant to survive (Bago \& Becard 2002). Hence, these fungi are classified as obligate symbionts. In addition, partners of a symbiosis can be termed as either the 
symbiont or the host. This is based on the relative size of each partner, with the larger partner usually being termed the host and the smaller one the symbiont. The symbiont may live outside the host, in which case the relationship is termed ectosymbiotic, or within the host, in which case the relationship is termed endosymbiotic.

Members of the phylum Cnidaria (e.g. sea anemones, corals, jellyfish) form obligate, endosymbiotic relationships with dinoflagellate algae of the genus Symbiodinium. Cnidarian-dinoflagellate symbioses have a worldwide distribution and are ecologically important members of many marine ecosystems. The most well known members of this group of symbiotic organisms are the scleractinian (or stony) corals (Figure 1.1). The dynamics of this relationship, which benefits both partners, provides the basis for a thriving reef community to develop in the nutrient poor waters of the tropical oceans (Stanley Jr \& van de Schootbrugge 2009). The ability to overcome resource limitation and the subsequent performance benefits, such as that seen in the cnidarian-dinoflagellate and AMF associations, is likely one of the key factors in driving the evolution and ecological success of such symbiotic relationships (Stat et al. 2006). 


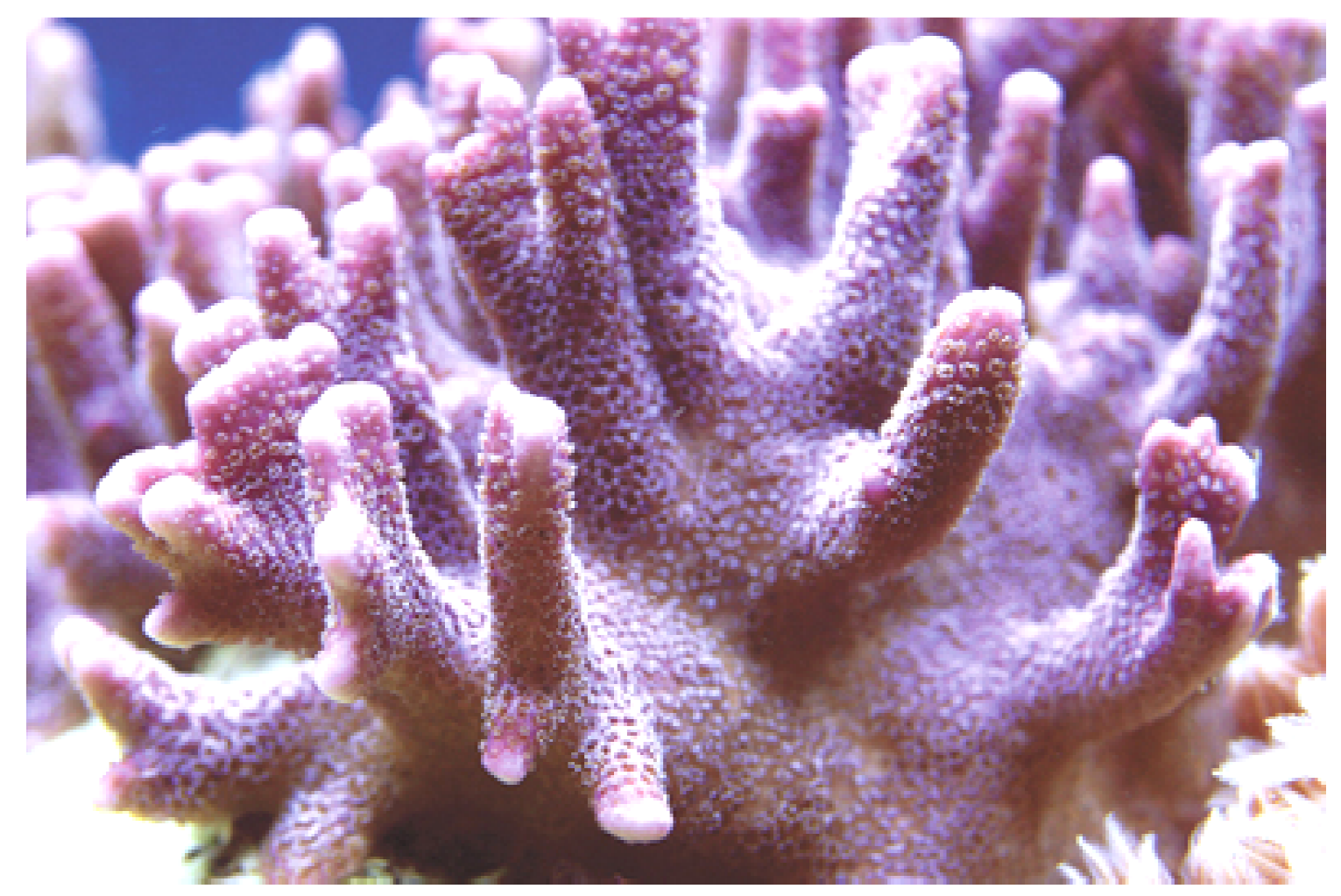

Figure 1.1: A well known and ecologically important example of an obligate, endosymbiotic relationship is the relationship formed between stony corals (Scleractinia) and dinoflagellates (Symbiodinium spp.), here exemplified by the coral Montipora digitata. The relationship is beneficial to both partners and provides the foundation for the development of highly biodiverse coral reef ecosystems. (Image: James Fatherree).

\subsection{Cnidarian-dinoflagellate symbiosis}

Dinoflagellate algae of the genus Symbiodinium form mutualistic symbioses with a range of cnidarians, including sea anemones, jellyfish and corals. The nutritional interactions between host and symbiont are of central importance to the ecological success of the cnidarian-dinoflagellate symbiosis. The following sections explore the ecological significance, structure, diversity and specificity of this symbiosis, the key nutritional interactions that enable its widespread success, and finally, how the symbiosis responds to environmental change. 


\subsubsection{Ecological significance}

Although cnidarian-dinoflagellate symbioses exist across a range of latitudes, the symbiosis is particularly important in the oligotrophic waters of the tropics, where coral reef ecosystems arise. Coral reefs are built predominantly by the action of scleractinian corals, which form hard calcium carbonate skeletons that constitute the physical reef structure. Coral calcification is greatly enhanced by symbiont photosynthesis, via a mechanism termed light enhanced calcification (LEC) (Vandermeulen et al. 1972, Davy et al. 2012). In fact, in the absence of symbionts, corals could not calcify at a rate sufficient to build coral reefs as we know them today. Furthermore, the algal symbionts provide photosynthetic products to the host, leading to enhanced metabolism, growth, reproduction and survival of corals (Muscatine et al. 1984; Davies 1991), while the symbionts also drive a tight recycling system in which they utilise host waste products, transferring them back to the host for subsequent use (Wang \& Douglas 1997; Lipschultz $\&$ Cook 2002). These processes result in the availability of complex habitat and energy in the midst of highly oligotrophic seas, so enabling the success of coral reefs in the tropics.

Corals represent a highly diverse group of organisms with a world-wide distribution. Coral diversity is centred in the tropics, and is particularly high in the Indo-West Pacific, known as the Coral Triangle, and to a lesser extent, the Caribbean (Rosen 1988). Coral reefs in the Indo-West Pacific generally harbour about 80 coral genera, while this number drops to about 30 genera in the Carribbean (Rosen 1988). Outside of the tropics, coral diversity drops abruptly, and above a latitude of approximately $35^{\circ} \mathrm{N}$ or below $35^{\circ} \mathrm{S}$, symbiotic reef-building corals cease to exist (Rosen 1988). While coral reefs are undoubtedly the most significant ecological result of the cnidarian-dinoflagellate symbiosis, numerous other cnidarian-dinoflagellate symbioses exist across a range of latitudes. For example, many sea anemones (actinians) also form symbioses with a range of Symbiodinium spp. Symbiotic sea anemones occur at both tropical and temperate latitudes, from $60^{\circ} \mathrm{N}$ in Alaska to $45^{\circ} \mathrm{S}$ in New Zealand (Buddemeier \& Fautin 1996). While symbiotic sea anemones are more prevalent in the tropics, they appear to be more locally dominant and have greater ecological importance in the temperate regions (Muller-Parker \& Davy 2001). In conclusion, cnidarian-dinoflagellate symbioses have a world-wide distribution and are ecologically important members of numerous marine ecosystems. 


\subsubsection{Structure, diversity and specificity}

\subsubsection{Structure}

The cnidarian body plan is simple and made up of just two cell layers, the epidermis and the gastrodermis (Venn et al. 2008). In corals and anemones, these cell layers take the form of a polyp, consisting of a body stalk (column) that encompasses the gastrodermal cavity and a ring of tentacles at the top of the column that encircles the single entrance to the gastrovascular cavity (Figure 1.2 A). Symbiodinium are brown coccoid dinoflagellates of approximately $10 \mu \mathrm{m}$ in diameter (Figure $1.2 \mathrm{~B}$ ). Typically, the cnidariandinoflagellate symbiosis is endosymbiotic, with the dinoflagellate symbiont cells residing within the host gastrodermal cells (Wakefield \& Kempf 2001). Symbionts occur at a density of approximately two algae per host cell (Muscatine et al. 1998). The symbionts are bound by the symbiosome membrane complex, which is composed of a host-derived outer layer and several symbiont-derived internal layers (Wakefiel \& Kempf 2001; Kazandjian et al. 2008). While the external appearance of Symbiodinium is relatively homogenous, there is in fact enormous diversity in the physiology and biochemistry of different Symbiodinium types, including the size, number and arrangement of chloroplasts (Blank \& Trench 1985; LaJeunesse 2001), photoadaptive physiology (Blank \& Trench 1985; Iglesias-Prieto \& Trench 1994), the production of mycosporine-like amino acids (Banaszak et al. 2000), isoenzyme profiles (Schoenberg \& Trench 1980; Blank \& Trench 1985), and the composition of fatty acids and sterols (Blank \& Trench 1985). 


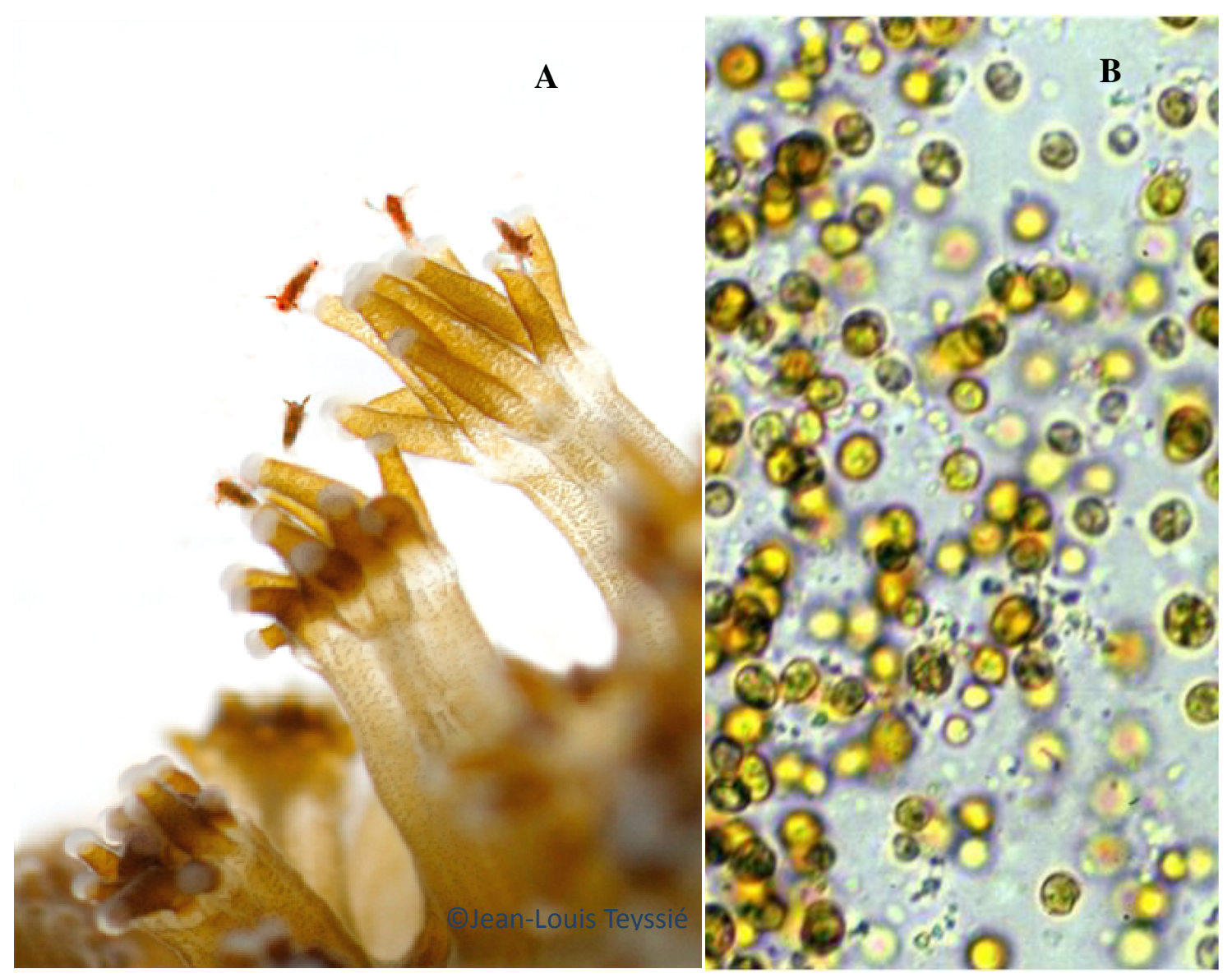

Figure 1.2: Typical structure of both partners involved in the cnidarian-dinoflagellate symbiosis. (A) Coral polyps depicting the column (lower portion), which encompasses the gastrovascular cavity, and the tentacles (upper portion), which encircle the single entrance to the gastrovascular cavity. Symbiodinium cells that reside within the host gastrodermal cells can also be seen as brown speckles (Image: Jean-Louis Teyssié). (B) Symbiodinium cells are light brown coccoid shaped cells of approximately $10 \mu \mathrm{m}$ in diameter (Image: Scott R. Santos, Dept. Biological Sciences, Auburn University).

\subsubsection{Diversity}

The cnidarian-dinoflagellate symbiosis is a highly diverse association. A large degree of genetic variation exists within the Symbiodinium genus (See Baker 2003; Coffroth \& Santos 2005; Stat et al. 2006, for reviews). Based on RFLP studies and 18S rDNA sequencing the diversity within the Symbiodinium genus is equivalent to that seen between different orders of free-living dinoflagellates (Rowan \& Powers 1992; Rowan 1998). The Symbiodinium genus can be divided into nine clades from A to I based on 
nuclear rDNA sequences (Rowan \& Powers 1991; LaJeunesse 2001; Pochon et al. 2004, Pochon and Gates, 2011). Diversity within each of these clades can be further defined based on the internal transcribed spacer 2 (ITS2) region of the rDNA. It is common practice to define Symbiodinium types according to ITS2 type (LaJeunesse 2001; Pochon et al. 2004). Clade $\mathrm{C}$ in particular associates with the widest range of host species and also contains the most diverse assemblage of ITS2 types (LaJeunesse 2005). While a wide range of host - Symbiodinium ITS2 type combinations exist, not all Symbiodinium types form stable symbiotic relationships with all hosts. The occurrence of different hostsymbiont combinations is affected by dispersal, environmental factors (such as depth and light) and host-symbiont specificity (Rowan \& Knowlton 1995; Loh et al. 2001; LaJeunesse 2002; Rodriguez-Lanetty et al. 2004; Sampayo et al. 2007; LaJeunesse et al. 2010, Baker 2003).

\subsubsection{Host-symbiont specificity}

Host-symbiont specificity (or lack thereof) refers to the tendency of both host and symbiont to associate with only one or a select few partners (specialists), or with a wide range of partners (generalists) (Baker 2003). Three broad phases are required for the establishment of a stable host-symbiont combination. This starts with host-symbiont recognition, followed by engulfment and then stable persistence of the symbiosis (Davy et al. 2012). Symbionts may be acquired either via maternal transmission (Davy \& Turner 2003), or more commonly, from the surrounding environment (Babcock et al. 1986).

Recognition is an important step in the selection ('winnowing') of potential symbionts, which ultimately gives rise to the final host-symbiont combination (Davy et al. 2012). Recent research has explored the potential role of glycan-lectin signalling in the recognition phase of symbiosis establishment. For example, Logan et al. (2010) used flow cytometry to characterise the cell-surface glycan assemblage of a number of Symbiodinium types. They found complex yet similar cell-surface glycan assemblages between types and concluded that glycan profiles may play a role in host-symbiont specificity (Logan et al. 2010). Furthermore, Markell \& Wood-Charlson (2010) detected large glycoproteins exuded by Symbiodinium cells and confirmed that these have a potential role as signalling molecules during host-symbiont recognition. 
Interestingly, the Symbiodinium type harboured by a host can vary both spatially and temporally. For example, Howe (2013) detected both seasonal and latitudinal variations in the symbiont composition of the temperate sea anemone Anthopleura aureoradiata. Due to the different biochemical and physiological characteristics of different symbiont types, different host-symbiont pairings may also differ from a functional perspective. Loram et al. (2007) explored this concept in the giant sea anemone (Condylactis gigantea) Symbiodinium association. They found that the nutritional contribution of symbionts, and how this varied in response to heat stress, was determined by the proportion of different symbiont types inhabiting the host (Loram et al. 2007). This study provides a clear demonstration that different host-symbiont combinations, which are at least partly driven by mechanisms involved in host-symbiont specificity, affect the response of cnidariandinoflagellate symbioses to environmental change.

\subsubsection{Nutritional exchange between partners}

Nutritional interactions in the cnidarian-dinoflagellate symbiosis are of central importance to the ecological success of the relationship. Nutritional interactions of particular importance include the translocation of photosynthetically-derived compounds (photosynthate), and the uptake, transport and utilization of nitrogen and phosphorus. These interactions are summarized in (Figure 1.3) and discussed in the following sections. 


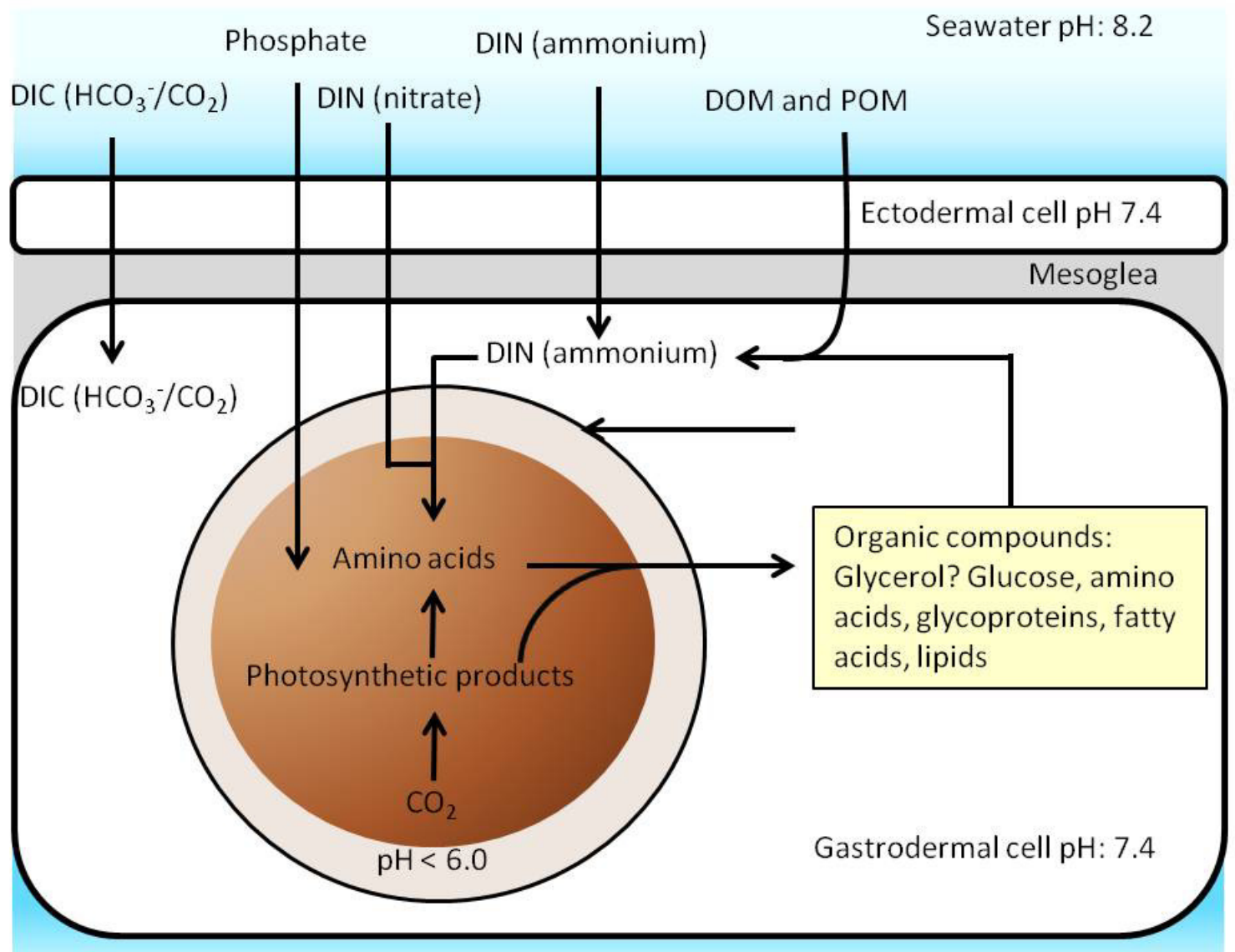

Coelentron $\mathrm{pH}: 9.0$

Figure 1.3. Nutritional interactions in the cnidarian-dinoflagellate symbiosis. Dissolved inorganic carbon (DIC) is transported from the external seawater to the symbiont for use in photosynthesis. Some of the photosynthetic products are then translocated to the host directly, or used in the synthesis of amino acids in conjunction with dissolved inorganic nitrogen (DIN) in the form of ammonium (host derived) or nitrate (from seawater). A portion of these amino acids may be translocated to the host. Phosphate is also transported from external seawater to the symbiont. Products from the host pool of organic compounds may be 'reverse translocated' back to the symbiont, while others may be degraded contributing to the host pool of ammonium. Dissolved organic matter (DOM), particulate organic matter (POM) and DIN (as ammonium) from external seawater also contribute to the host ammonium pool. Figure adapted from Gordon \& Leggat (2010); Davy et al. (2012). 


\subsubsection{Translocation of photosynthate}

As early as 1955, it was recognized that some form of 'coral herbivory' must be occurring in order to resolve the existing trophic structure of coral reef communities (Odum \& Odum 1955). In response to this suggestion, Muscatine \& Hand (1958) incubated symbiotic anemones under both light and dark treatments in the presence of $\mathrm{Na}_{2}{ }^{14} \mathrm{CO}_{3}$ for periods of 18 hours to 5 weeks. Subsequent autoradiographs of anemone tissue conclusively demonstrated uptake of the radioactive carbon by symbiotic dinoflagellates and its eventual translocation to host tissue. This seminal work provided the first direct experimental evidence for the transfer of photosynthetically fixed carbon from symbiont to host.

Following on from his earlier work, Muscatine (1967) incubated symbiotic dinoflagellates isolated from the giant clam Tridacna crocea and the coral Pocillopora damicornis in host homogenate in the presence of $\mathrm{Na}_{2}{ }^{14} \mathrm{CO}_{3}$. Subsequent analysis based on 2-D radiochromatography demonstrated that glycerol generally accounted for $>90 \%$ of the photosynthetically fixed carbon released into the extracellular medium; glycolate, glucose and a ninhydrin-positive compound were also present but in lower quantities. Glucose was identified as the major photosynthetically fixed compound retained within the algae, along with numerous photosynthetic intermediates and lipids. Alternatively, glycerol may comprise $24.8 \%$ to $95 \%$ of the photosynthate released, with glucose, alanine and various organic acids present in lower quantities (von Holt \& von Holt 1968; Trench 1971a; b, 1993; Sutton \& Hoegh-Guldberg 1990). An important issue to note here is that isolated symbionts do not necessarily behave the same as they do in hospite. For example, photosynthetic rates are likely altered (Sutton \& Hoegh-Guldberg 1990), as is membrane permeability (Steele \& Goreau 1977).

A number of more direct methods have been applied, which allow identification of compounds translocated from symbiont to host in the intact symbiosis. Lewis \& Smith (1971) applied the 'inhibition technique' to a range of symbiotic cnidarians. Here, the symbiotic entity is incubated in a medium containing $\mathrm{NaH}^{14} \mathrm{CO}_{3}$ along with a range of potential translocated compounds at concentrations sufficient to saturate all sites of uptake in the host. Thus, corresponding photosynthetically fixed ${ }^{14} \mathrm{C}$-labelled compounds released by the symbiont are released from the symbiosis entirely. The results indicated 
that glycerol, glucose and alanine were the major translocated compounds. More recently, Whitehead \& Douglas (2003) applied a similar method known as 'metabolite comparison' to the symbiotic anemone Anemonia viridis. Here, one group of organisms is incubated in the light in the presence of $\mathrm{NaH}^{14} \mathrm{CO}_{2}$ and another in the dark in the presence of candidate translocated ${ }^{14} \mathrm{C}$-labelled compounds. The metabolite profile of the host tissue is then compared between treatments and when metabolite profiles match, it is assumed that the exogenously supplied candidate compounds represent those translocated from symbiont to host. Their results indicated that glucose, succinate and fumarate were the major translocated compounds, and in contrast to previous studies they found no evidence for the translocation of glycerol.

Further evidence that glucose and not glycerol is the major translocated compound comes from Burriesci et al. (2012). In this study, intact symbiotic anemones (Aiptasia sp.) were incubated in the light in the presence of ${ }^{13} \mathrm{C}$-bicarbonate. Metabolite profiles generated by gas chromatography coupled to mass spectrometry (GC-MS) revealed ${ }^{13} \mathrm{C}$-labelled glucose but no glycerol translocated from the symbiont to host tissues within two minutes of exposure to ${ }^{13} \mathrm{C}$-bicarbonate. Additionally, when anemones were incubated in the presence of ${ }^{13} \mathrm{C}$-labelled glycerol, no accumulation of ${ }^{13} \mathrm{C}$-labelled glucose in host tissues was observed, suggesting that the presence of glucose was not a by-product of glycerol translocation. The authors also applied the same method to three species of corals and similar trends of ${ }^{13} \mathrm{C}$-labelled glucose production were also observed. Finally, Symbiodinium cells freshly isolated from Aiptasia were incubated in host homogenate along with ${ }^{13} \mathrm{C}$-bicarbonate. This resulted in the rapid release of ${ }^{13} \mathrm{C}$-labelled glycerol, indicating that trends observed in previous studies may be a by-product of experimental conditions. Interestingly, the pattern of photosynthate translocation observed by both Whitehead \& Douglas (2003) and Burriesci et al. (2012) is consistent with observations in the intercellular giant clam-Symbiodinium symbiosis, where glucose is translocated in the intact symbiosis and glycerol is released from the symbionts when incubated in host mantle homogenate (Ishikura et al. 1999)

Evidence also exists for a number of additional compounds being translocated between symbiont and host. For example, Markell \& Trench (1993) identified a range of glycoconjugates exuded from the cell surface of five species of cultured Symbiodinium. Additionally, Markell \& Wood-Charlson (2010) applied immunocytochemical techniques 
that enabled the identification of a number of glycoproteins secreted by Symbiodinium cells, both in culture and when in symbiosis with their host, the jellyfish Cassiopea xamachana. Although it has been suggested that the primary role of these compounds is in symbiont-host recognition, they also contribute to the pool of translocated photosynthetic products. Furthermore, evidence exists for 'reverse translocation' of compounds from the cnidarian host to the symbiotic algae. This includes translocation of the amino acid alanine (Carroll \& Blanquet 1984), phosphate (Deane \& O'Brien 1981), ammonium and nitrate (Domotor et al. 1984; Gunnersen et al. 1988), and various organic substrates (Steen \& Angeles 1987).

\subsubsection{Nitrogen and phosphorus dynamics}

An intricate system for the uptake and utilisation of nitrogen exists in the cnidariandinoflagellate symbiosis. Nitrogen sources include dissolved and particulate organic nitrogen (Cook et al. 1992; Ferrier-Pagès et al. 1998; Davy et al. 2006) and dissolved inorganic nitrogen (ammonium and nitrate) from the external seawater (Muscatine \& D'Elia 1978; Grover et al. 2002; Badgley et al. 2006), and ammonium produced in host catabolism (Cates \& Mclaughlin 1976; Rahav et al. 1989). Sources of nitrogen can be assimilated by both host and symbiont (Catmull et al. 1987; Dudler \& Miller 1988; Grover et al. 2002; Lipschultz \& Cook 2002), however it is believed that the symbiont is the primary site of nitrogen assimilation (Grover et al. 2002). In the host, nitrogen assimilation occurs mainly via the NADP-glutamate dehydrogenase (NADP-GDH) pathway (Miller \& Yellowlees 1989; Roberts et al. 2001), while in the symbiont it is via the glutamine synthetase/glutamine 2-oxoglutarate amido transferase (GS/GOGAT) pathway (Wilkerson \& Muscatine 1984; Summons et al. 1986; Roberts et al. 2001).

Interestingly, the $\mathrm{pH}$ of the symbiosome vacuole potentially plays an important role in the transport of ammonium from host to symbiont. Research suggests that the symbiosome vacuole is acidic, with a $\mathrm{pH}$ of $<6.0$ (Venn et al. 2009). As acidity increases, the chemical equilibrium of the reaction $\mathrm{H}_{3} \mathrm{O}^{+}+\mathrm{NH}_{3} \Leftrightarrow \mathrm{H}_{2} \mathrm{O}+\mathrm{NH}_{4}^{+}$is altered, resulting in an increase in the ammonium ion $\left(\mathrm{NH}_{4}{ }^{+}\right)$concentration. As opposed to ammonia $\left(\mathrm{NH}_{3}\right)$, these ammonium ions cannot permeate biological membranes, suggesting that this source of nitrogen must enter the symbiont via carrier-mediated transport. The alternative source of nitrogen, nitrate $\left(\mathrm{NO}_{3}{ }^{-}\right)$, can only be converted to ammonium by the symbiont, and not 
the host, for subsequent assimilation into amino acids. This requires both nitrate and nitrite reductases, and both have been identified in a number of Symbiodinium types (Crossland \& Barnes 1977; Leggat et al. 2007). Once inside the symbiont, nitrogen sources are assimilated via the GS/GOGAT pathway, and subsequently translocated back to the host as either free amino acids (Trench 1971b; Sutton \& Hoegh-Guldberg 1990; Wang \& Douglas 1997) or protein-rich glycoconjugates (Markell \& Trench 1993; Markell \& Wood-Charlson 2010), completing a tight 'nitrogen recycling' system between host and symbiont.

Numerous studies have recorded greatly elevated ammonium levels in the host in the absence of symbionts and this has been interpreted as support for this 'nitrogen recycling' hypothesis (Cates \& Mclaughlin 1976; Wilkerson \& Muscatine 1984). However, while the evidence for nitrogen recycling is compelling, a study by Wang \& Douglas (1998) supports an alternative explanation for the patterns of ammonium levels observed in the symbiosis, termed 'nitrogen conservation' These authors suggest that the increased levels of host ammonium observed in the absence of symbionts are in fact caused by reduced ammonium assimilation into nitrogenous compounds, and increased ammonium production as a result of amino acid degradation in the absence of symbiont-derived carbon compounds. While controversy still abounds regarding 'nitrogen recycling' versus 'nitrogen conservation', nitrogen dynamics clearly remain essential to the ecological success of the cnidarian-dinoflagellate symbiosis.

Phosphorus is another essential nutrient in the cnidarian-dinoflagellate symbiosis. Sources include dissolved inorganic phosphorus (as phosphate, $\mathrm{PO}_{4}{ }^{3-}$ ) and particulate organic phosphorus (e.g. zooplankton). Numerous studies suggest that phosphate uptake is primarily driven by the algal symbiont (Pomeroy \& Kuenzler 1969; D'Elia 1977; D'Elia et al. 1983; Muller-Parker et al. 1990; Godinot et al. 2009). In fact, two acid phosphatases (P-1 and P-2) have previously been identified in Symbiodinium cells isolated from the coral Acropora formosa (Jackson et al. 1989). In this study, P-1 was found to be particularly active on polyphosphate, suggesting that it may be involved in mobilizing phosphate stored in this form. The activity of P-2 was unresolved, though these authors suggested that it may be involved in hydrolyzing phosphate esters external to the algal cell membrane prior to uptake by the algal transport system. In contrast, the uptake kinetics of phosphate in the coral Stylophora pistillata and its symbiotic dinoflagellates 
provide compelling evidence for the presence of phosphate transporters in both host and symbiont (Godinot et al. 2009).

\subsubsection{Response to environmental stressors: biochemical and physiological adjustments}

A range of environmental stressors are known to impact the cnidarian-dinoflagellate symbiosis, including high and low temperatures, nutrients, pollution and light (Lesser 2006). Some of these stressors may also act in synergy, greatly increasing the detrimental impact to the organism. For example, Coles \& Jokiel (1978) found that high light and high temperature have a synergistic detrimental impact on the coral Montipora verrucosa. In particular, high light at already elevated temperatures caused increased loss of symbiont photosynthetic pigments, reduced carbon fixation, reduced growth rates and increased coral mortality.

These environmental stressors cause a wide range of alterations at the cell and molecular levels, which if not managed appropriately lead to cellular dysfunction and damage. Common cellular and molecular impacts caused by environmental stressors include altered osmotic pressure, the build up of toxic metals and inorganic ions, changes to membrane structure and function, altered energy requirements and availability, increased levels of reactive oxygen species (ROS), and direct or secondary damage to nucleic acids, proteins and enzymes (Kültz 2005; Wahid et al. 2007; Guy et al. 2008; Abele 2011). The production of ROS appears to be a particularly prominent outcome of environmental stress in both partners of the cnidarian-dinoflagellate symbiosis (Dykens et al. 1992; Nii \& Muscatine 1997; Franklin et al. 2004; Lesser \& Farrell 2004). While there are many similarities between the cellular and molecular impacts of environmental stress between species (Sulmon et al. 2015), environmental stressors do in fact impact both partners of the cnidarian-dinoflagellate symbiosis differentially. For instance, Nii \& Muscatine (1997) demonstrated that thermally-induced oxidative stress in the symbiotic sea anemone Aiptasia pulchella is primarily an animal response, caused by damage to host mitochondria and consequent superoxide ion production. That being said, due to the interrelated nature of the cnidarian-dinoflagellate symbiosis, environmental stressors 
beyond threshold levels will eventually affect the functioning of both partners, and the symbiosis as a whole (Clark \& Jensen 1982).

A number of mechanisms that are likely to be involved in the attenuation of cellular and organismal damage have been recorded in both partners of the cnidarian-dinoflagellate symbiosis. For example, Kneeland et al. (2013) recorded reduced polyunsaturated fatty acid (PUFA):saturated fatty acid and total fatty acid:sterol ratios in Symbiodinium cells exposed to heat stress over a period of weeks. They suggested that the overall decline in fatty acid ratios was driven by a reduction in the unsaturated fatty acid content of membranes at elevated temperatures. The PUFA content of cell membranes influences their fluidity, which in turn plays an important role in determining the cellular impact of thermal stress (Stubbs \& Smith 1984). Interestingly, Garrett et al. (2013) documented altered lipid profiles of the tropical sea anemone Aiptasia pallida in its symbiotic versus aposymbiotic state. As the symbiont population density is impacted by environmental conditions, it is likely that host lipid profiles would also be influenced. Heat shock proteins are another mechanism that Symbiodinium cells may employ to tolerate extreme temperatures. For example, Rosic et al. (2011) recorded an increase in Hsp70 gene expression from $39 \%$ to $57 \%$ in the symbionts of the coral Acropora millepora when exposed to moderate to severe heat stress. An interesting body of research has also demonstrated that nutrient limitation (with a particular emphasis on nitrogen) can lead to significantly reduced photosynthetic rates and carbon fixation (Cook et al. 1992; Davy et al. 2006; Morar et al. 2011), presumably resulting in reduced translocation of photosynthetic products from symbiont to host. Further research by Kopp et al. (2013) in the symbiotic coral Pocillopora damicornis, revealed that the assimilation of nitrogenous compounds by the symbiotic algae was followed by $\mathrm{N}$ storage as uric acid crystals, followed by $\mathrm{N}$ remobilisation accompanied by the translocation of nitrogenous compounds from symbiont to host. This provides evidence for a mechanistic basis by which nutrient limitation may result in reduced nutritional flux from symbiont to host. In other photosynthetic organisms such as Arabadopsis thaliana, responses to extreme thermal challenge include increased pools of amino acids, compatible solutes and polyamine precursors (Kaplan et al. 2004). Of particular interest, a large scale review of the metabolic response of plants to temperature stress revealed a common role of central carbohydrate metabolism (Guy et al. 2008). Results reported by Hillyer et al. (2015) in 
the cnidarian-dinoflagellate symbiosis indicate that this response may also be shared by Symbiodinium spp.

Cnidarian hosts also possess a range of mechanisms to overcome the detrimental effects of environmental stress. Two prominent antioxidants employed by cnidarians include super oxide dismutase (SOD) and catalase (CAT). These antioxidants act to neutralise the reactive oxygen species, superoxide $\left(\mathrm{O}_{2}{ }^{-}\right)$and hydrogen peroxide $\left(\mathrm{H}_{2} \mathrm{O}_{2}\right)$, respectively, which are produced as a result of heat- and light-stress (Richier et al. 2005; Levy et al. 2006; Weis 2008). Compatible solutes have also been evoked as important compounds in the cnidarian stress response. Mayfield \& Gates (2007) proposed that the compatible solutes glycerol and free amino acids (FAAs) may be particularly important in the cnidarian response to environmental stress. These authors postulated that damage to the symbiont photosynthetic apparatus as a result of temperature, light, salinity or pollutants results in reduced translocation of glycerol and FAAs from the symbiont, leading to osmotic stress within host cells and the eventual breakdown of the symbiosis. Cnidarians may also alter basal energy metabolism in order to reduce damage caused by environmental stress. For example, many marine invertebrates respond to temperature stress by reducing mitochondrial density and increasing mitochondrial proton leakage (Abele 2011). This has the effect of reducing thermally-accelerated oxygen consumption, with a concomitant production of ROS, but leads to energetic restriction (Abele 2011). Direct evidence of altered energy metabolism in Cnidaria comes from a study by Leggat et al. (2011), who detected up-regulation of a suite of genes involved in carbon metabolism, including glyceraldehyde-3-phosphate dehydrogenase, alpha-ketoglutarate dehydrogenase, glycogen synthase and glycogen phosphorylase in the coral Acropora aspera in response to increased temperature.

Above threshold levels, the mechanisms employed to deal with environmental stress become overwhelmed, leading to cellular and organismal dysfunction. Particular attention has been paid to the sensitivity of the cnidarian-dinoflagellate symbiosis to heat- and light-stress, especially in tropical associations in which slight variations in these stressors can lead to a rapid degradation of health and performance. Numerous studies have documented temperature- or light-induced reductions in photosynthetic efficiency, carbon fixation, translocation and coral growth rate, along with host DNA damage and cell apoptosis or necrosis (Coles \& Jokiel 1978; Clark \& Jensen 1982; Lesser \& Farrell 2004). 
Extreme stress results in breakdown of the symbiotic relationship and expulsion of the symbiotic algae, in a process known as coral bleaching (Weis 2008). Coral bleaching results in reduced growth, reproduction and eventual mortality if not rapidly ameliorated. Interestingly, tropical cnidarian-dinoflagellate symbioses appear to be much more sensitive to bleaching than their temperate counterparts (Muller-Parker \& Davy 2001).

While some of the mechanisms employed to overcome environmental stress in the cnidarian-dinoflagellate symbiosis have been determined, many intricate details and an understanding of the differences between species are still lacking. For example, what processes underlie the more robust nature of temperate cnidarian-dinoflagellate symbioses in the face of large fluctuations in environmental conditions?

\subsection{Temperate cnidarian-dinoflagellate symbioses}

Cnidarian-dinoflagellate symbiosis in temperate regions is largely represented by the relationship between sea anemones (actinians) and Symbiodinium. In the Pacific basin, temperate cnidarian-dinoflagellate symbioses occur as far north as Alaska $\left(60^{\circ} \mathrm{N}\right)$ and as far south as New Zealand $\left(45^{\circ} \mathrm{S}\right)$ (Buddemeier \& Fautin 1996). They are also known to occur in the Atlantic basin in the east and west of Europe, as far north as Scotland $\left(55^{\circ} \mathrm{N}\right)$ (Roberts et al. 1999). Current knowledge suggests that at these temperate locations they are ecologically important and abundant members of the marine ecosystem (MullerParker \& Davy 2001). It has been suggested that this may be due to photosyntheticallyfixed carbon enhancing sexual reproduction and growth of the host, which in turn provides a competitive advantage over other non-symbiotic invertebrates (Davy et al. 1997; Muller-Parker \& Davy 2001).

Our current knowledge indicates that symbiotic algae hosted by anemones in temperate regions belong mostly to Symbiodinium clade A, with a smaller portion belonging to clade B. Clade B Symbiodinium has been documented in the symbiotic sea anemone Anthopleura elegantissima on the Pacific coast of the USA (LaJeunesse \& Trench 2000). Additionally, Visram et al. (2006) recorded one sea anemone, Bunodeopsis strumosa, that harboured clade B symbionts, while nine of the other associations they tested from the Mediterranean sea harboured clade A symbionts. Clade A symbionts were also recorded in 
the sea anemone Anemonia viridis, occurring in the southwest of England (Bythell et al. 1997). More recently, Howe (2013) identified two Symbiodinium ITS2 types belonging to clade A ('Med clade A' and 'Amed') in the symbiotic sea anemone, Anthopleura aureoradiata, across a broad latitudinal range in New Zealand. As genetically different Symbiodinium types possess different biochemical and physiological traits, symbiotic associations involving different symbiont types are likely to respond differently to environmental conditions. This has been shown to play an important role in determining what symbiont types are harboured by cnidarian hosts in regions exposed to different environmental regimes (LaJeunesse \& Trench 2000; Thornhill et al. 2008).

In general, temperate symbioses are exposed to much larger fluctuations in environmental parameters on a seasonal basis than are their tropical counterparts (Muller-Parker \& Davy 2001). Some important environmental parameters that appear to be particularly variable in temperate, as opposed to tropical regions, include temperature and irradiance, nitrogen, ammonium and phosphorus concentrations, and phytoplankton abundance. More specifically, in temperate regions, temperature and irradiance are generally lower than in the tropics, though the magnitude of change between seasons is much larger. In contrast, temperate waters contain much higher nitrogen, ammonium, phosphate and chlorophyll $a$ levels than do tropical waters, but these again fluctuate drastically between seasons; tropical regions are relatively stable in this regard (Muller-Parker \& Davy 2001).

These differences in environmental conditions appear to affect the symbiotic relationship in a number of ways. For example, the "contribution of zooxanthellae to animal respiration' (CZAR) has been reported as being less than $90 \%$ in most temperate associations due to the limited availability of light, while the CZAR generally exceeds $100 \%$ in most tropical associations (Muller-Parker \& Davy 2001). Of particular relevance to this thesis, symbiont densities in temperate symbioses persist stably under the large environmental fluctuations to which they are exposed (Dimond et al. 2011), while symbiont densities in tropical symbioses vary dramatically between seasons and in response to stress (Fitt et al. 2000; Gabriel \& Rodney 2006; LaJeunesse et al. 2009; see above). In extreme cases, this leads to coral bleaching, which poses a major threat to the long term persistence of coral reef ecosystems (Hoegh-Guldberg 1999; Hughes et al. 2003; Hoegh-Guldberg et al. 2007, 2011; Veron et al. 2009). While it is well known that different symbiont types influence the response of the symbiosis to environmental 
variability, the metabolic processes underlying the relative stability of temperate cnidarian-dinoflagellate symbioses has received little attention to date.

\subsection{Metabolomics}

Research to date has elucidated a number of the core nutritional interactions in the cnidarian-dinoflagellate symbiosis. A clear picture is also forming regarding how the symbiosis is impacted by, and responds to, environmental variability, in the form of both abiotic (e.g. heat and light) and biotic (e.g. symbiont type) factors. This includes a suite of biochemical and physiological adjustments, such as altered nutritional support from symbiont to host, the production of specific metabolites to counter damage, and changes to a number of metabolic processes (e.g. energy production) in both host and symbiont (see above). While this information is important, organismal responses to environmental variability do not just involve metabolic alterations in isolation, but rather a dynamic remodelling of the entire metabolic network. Although some progress has been made regarding the response of the cnidarian-dinoflagellate symbiosis to environmental variability, our knowledge regarding changes to individual metabolites and metabolic pathways, and the interrelated nature of these changes, remains limited. Nevertheless, the application of advanced metabolomics techniques, such as have recently been employed by Burriesci et al. (2012) and Hillyer et al. (2015), has the potential to greatly enhance our understanding of these dynamic processes in the cnidarian-dinoflagellate symbiosis.

\subsubsection{Concepts and approaches}

The metabolome is defined as the entire complement of low molecular weight molecules present in a cell at any point in time (Oliver et al. 1998). Metabolite profiling involves the generation of unbiased, quantitative profiles of a specific set of metabolites contributing to this metabolome (Fiehn 2001). These metabolites may be of a specific class, such as carbohydrates, amino acids or lipids, or components of a metabolic pathway of interest. A range of technologies exist for metabolite identification, including gas chromatography coupled to mass spectrometry (GC-MS), liquid chromatography coupled to mass spectrometry (LC-MS), Fourier transform-infrared spectrometry (FT-IR) and nuclear magnetic resonance (NMR) (Saito \& Matsuda 2010). Different techniques are suited to 
identifying different metabolites, such as volatile versus non-volatile compounds, or large versus small molecules.

GC-MS provides a particularly powerful platform for a broad range of metabolites involved in primary metabolism, including organic acids, amino acids, sugars, sugar alcohols, phosphorylated intermediates, and lipophilic compounds such as fatty acids (Lisec et al. 2006). Of all the metabolomic techniques, GC-MS offers an ideal combination of reliability, resolution and sensitivity for exploring the core pathways of primary metabolism (Fiehn et al. 2000; Lisec et al. 2006). One disadvantage of GC-MS, though, is that many important polar metabolites, such as those containing alcohol, phenol, carboxylic acid, amine and amide functional groups are not inherently volatile enough for direct analysis. However, these metabolites can be chemically derivatised to increase volatility and hence enable processing via this method (Fancy \& Rumpel 2008).

Once data have been generated, metabolite profiles of samples from different conditions can be compared using multivariate statistics, such as principal components analysis (PCA) or partial least squares discriminant analysis (PLS-DA) (Bundy et al. 2008). These multivariate approaches enable the relative importance of different metabolites to any environmentally-induced response to be determined, and hence allow inferences about the functional roles of these metabolites. Further analysis of these metabolite profiles enables determination and comparison of the activity levels of different metabolic pathways between environmental conditions. One such method, termed pathway activity profiling (PAPi), employs an algorithm to calculate pathway activity scores based on the relative abundance of metabolites and their involvement in metabolic pathways based on information stored in the Koyoto Encyclopedia of Genes and Genomes (KEGG) database (Aggio et al. 2010; Booth et al. 2013).

\subsubsection{Application to the study of symbiosis}

The recent application of metabolomics to the study of symbioses such as that between plants and arbuscular mycorrhizal fungi (AMF), highlights the potential of this approach to answer fundamental questions about the cnidarian-dinoflagellate symbiosis. The plant- 
AMF symbiosis has some pertinent similarities with the cnidarian-dinoflagellate symbiosis, being an endosymbiotic relationship with a smaller symbiont (the fungus) growing within cells of a larger host (the plant) (Figure 1.4); furthermore, both symbioses are based on nutritional exchange between the two partners. An excellent example of how metabolomics has elucidated the plant-AMF association is the work of Schliemann et al. (2008), who generated metabolite profiles of the roots of barrel medic (Medicago truncatula) both in and out of symbiosis with the fungus Glomus intraradices, under differing levels of external phosphate availability. These authors observed AM-dependent increases in a range of metabolites, including amino acids and fatty acids, along with the stimulation of the biosynthesis of a number of isoflavonoids; an accumulation of fungusspecific fatty acids was also recorded. Furthermore, PCA was able to clearly define between AMF- vs. non-AMF-associated roots. A similar study by Rasmussen et al. (2008) revealed significant differences in the quality and quantity of host metabolites in the blades of rye-grass (Lolium perenne) that were infected with three different strains of the fungus Neotyphodium lolii, and exposed to two different levels of exogenous nitrogen. These studies clearly demonstrate the power of metabolomics in determining adjustments to metabolic processes of endosymbiotic associations in response to different environmental conditions, and a recent authoritative review of the cell biology of cnidarian-dinoflagellate symbiosis called for the application of such methods to clarify important questions regarding metabolic processes in that system (Davy et al. 2012). 


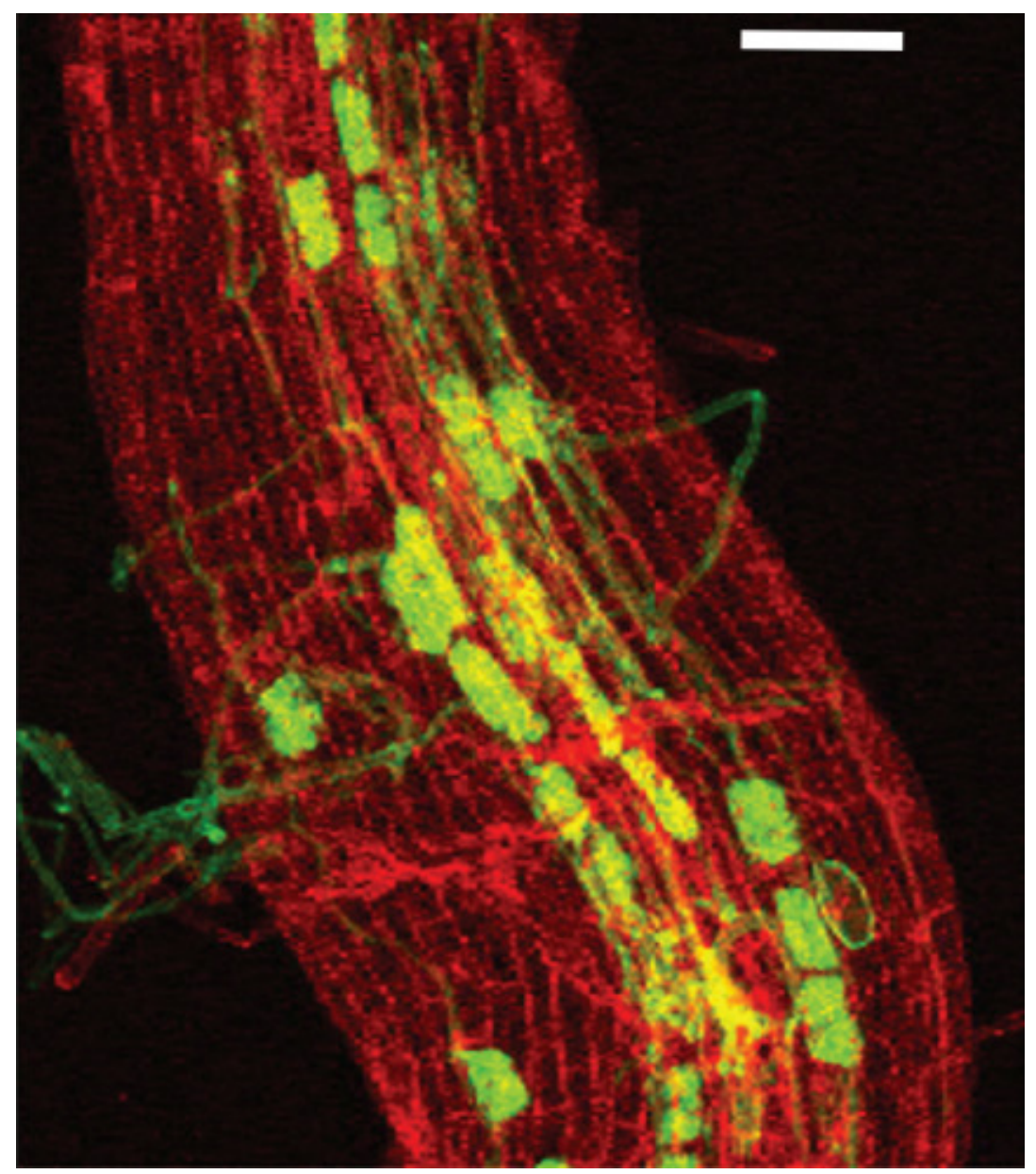

Figure 1.4: Confocal laser-scanning microscopy image of fluorescently-stained mycorrhizal fungus associated with the roots of white clover. Red stain indicates plant cells, while green stain indicates mycorrhizal fungus. The large green regions are mycorrhizal abruscules within plant root cells. Scale bar is $100 \mu \mathrm{m}$. Adapted from Vierheilig et al. (2005) (Image: A. Hirsch).

Recently, reliable high-throughput methods suitable for GC-MS analysis of both partners of the cnidarian-dinoflagellate symbiosis have been developed (Roessner et al. 2000; Villas-Bôas et al. 2006; Burriesci et al. 2012; Hillyer et al. 2015). Of particular relevance, Hillyer et al. (2015) employed GC-MS to generate metabolite profiles of both host and symbiont of the association formed between the tropical sea anemone Aiptasia sp. and its homologous dinoflagellate symbiont, Symbiodinium minutum, in response to elevated temperature. The authors observed changes in a range of metabolites associated with core metabolic processes, which were also reflected in the separation of metabolite profiles via 
PCA analysis. The metabolite profiles were also subject to PAPi analysis, which revealed alterations to numerous central metabolic pathways, particularly those involved in carbohydrate and energy metabolism.

In parallel to the developments of metabolomics to symbiotic associations, the field of 'environmental metabolomics' has been rapidly expanding due to the power of metabolomics to reveal novel biochemical mechanisms employed by organisms in response to a wide range of environmental stressors (Motti 2012; Lankadurai et al. 2013). The recent advances in both fields of study have opened the door for many important and hitherto unknown aspects of the metabolic response of the cnidariandinoflagellate symbiosis to environmental change to be explored. For example, we still know relatively little about how metabolic pathways in the symbiosis respond to many environmental factors, including temperature, light and nutrient availability. This knowledge gap is particularly relevant given that tropical associations are especially sensitive to environmental fluctuations, and that mass coral bleaching events are expected to increase in severity over the coming decades. In addition though, metabolomics provides a means of elucidating the physiological basis of environmental resilience in temperate cnidarian-dinoflagellate symbioses. Comparison between the responses of temperate and tropical symbioses has the potential to shed light on the mechanisms underlying thermal sensitivity versus resilience in the cnidarian-dinoflagellate symbiosis, and environmentally sensitivity versus resilience more generally in this association.

In this thesis, state-of-the-art metabolomics technologies were applied to characterise the metabolic response of a temperate cnidarian-dinoflagellate symbiosis to environmental variability. More specifically, metabolite profiles and metabolic pathway activities were compared between seasons in the field and in response to differing thermal regimes in the laboratory, to improve our understanding of how environmental variability affects metabolic processes in this robust system. This in turn provided insight into the mechanisms that enable the persistence of such associations under highly variable environmental conditions, and provided a model against which comparisons with more sensitive tropical associations can be made. 


\subsection{Aims and objectives}

The aim of this thesis was to determine the impact of environmental change on the metabolic functioning of both partners in a temperate cnidarian-dinoflagellate symbiosis, formed between the common New Zealand sea anemone Anthopleura aureoradiata and Symbiodinium sp.

The specific objectives were:

Objective 1 - To determine whether there is a difference in the metabolite profile between seasons in either the host or symbiont from anemones sampled in situ; and to identify the changes in the specific metabolites and metabolic pathways that account for these differences.

Hypothesis 1 - Metabolic processes will differ between summer and winter in both the host and symbiont. These differences will be driven by increases in the abundance of metabolites important in thermal resistance, and the up-regulation of metabolic pathways associated with the maintenance of cellular homeostasis, e.g. those involved in carbon fixation (symbiont only), respiration and growth.

Objective 2 - To determine the impact of long term exposure to extreme temperatures (low and high) on metabolic processes in both the host and symbiont via laboratory experimentation; and to identify the changes in specific metabolites and metabolic pathways that account for any observed changes.

Hypothesis 2 - Metabolic processes will differ in anemones exposed to high and low temperatures relative to controls, in both the host and symbiont. This pattern will reflect the patterns observed in the field between summer and winter, with cold-treated and heat-treated samples showing increases in the abundance of metabolites important in cold or heat tolerance, respectively. Metabolic pathways associated with the maintenance of cellular homeostasis e.g. those involved in carbon fixation (symbiont only), respiration and growth are expected to show a decline in activity in cold-treated samples and an increase in heat-treated samples. 


\section{Chapter 2: Materials and Methods}

\subsection{Seasonal comparison}

\subsubsection{Study site and sampling design}

Anemones for a seasonal comparison study were collected from the intertidal zone on the western side of Point Halswell, the northern most point of Kau Bay within Wellington Harbour $\left(41^{\circ} 17^{\prime} 02^{\prime \prime} \mathrm{S}, 174^{\circ} 49^{\prime} 33^{\prime \prime} \mathrm{E}\right)$. The study site receives all day sunlight and is exposed to winds from the west through to the northeast. The terrain is characterised by numerous cracks and rock pools, and a sandstone substrate. The site displays typical intertidal zonation (Stephenson \& Stephenson 1949) with an infralittoral fringe dominated by brown macroalgae, a midlittoral zone dominated by barnacles, whelks and limpets, and a supralittoral fringe dominated by littorinid snails. The study species, Anthopleura aureoradiata, is distributed throughout the midlittoral zone, but occurs most densely in the upper half of this zone.

Anemones were sampled from the upper midlittoral zone on the 10/10/2014, hereafter referred to as "winter" and on the 07/03/2015, hereafter referred to as "summer". A $50 \mathrm{~m}$ transect was set up parallel to the shore within the midlittoral zone. Ten sites were selected at random along this transect and sampled on each of the two collection dates. Each site was approximately $5 \mathrm{~m}$ apart to reduce the possibility of sampling clones. All samples were taken during a midday low tide to ensure that aerial exposure, diel cycle variations and the angle of incidence of sunlight remained constant between samples (Yellowlees et al. 2008). Sampling of individuals was carried out using methods modified from (Hines et al. 2007; Lisec et al. 2006). At each sampling point a crack exposed to direct sunlight and air was chosen. Individuals were prised off the rocks using bluntended stainless steel tweezers that were rinsed first with ethanol and then with FSW between samples. Once detached from the rocks, anemones were rinsed using FSW to remove as much debris as possible, placed in a cryovial and immediately frozen in liquid nitrogen $\left(\mathrm{LN}_{2}\right)$. This entire process took under one minute to complete per anemone. Samples were then transferred to a $-80^{\circ} \mathrm{C}$ freezer and stored ready for analysis of host protein content, Symbiodinium cell density, chlorophyll $a$ content and host and symbiont metabolite profiles. 


\subsubsection{Environmental conditions}

Environmental conditions were recorded for two weeks prior to each sampling date. Maximum daily gust $(\mathrm{km} / \mathrm{h})$ and wind direction, daily rainfall $(\mathrm{mm})$, daily maximum and minimum temperatures $\left({ }^{\circ} \mathrm{C}\right)$, and average daily radiation intensity $\left(\mathrm{MJ} / \mathrm{m}^{2}\right)$ were obtained from CliFlo: NIWA's National Climate Database (National Institute of Water and Atmospheric Research 2015). Seawater samples were also collected during high tide on four dates during the two weeks leading up to each sampling event. These were analysed for a suite of nutrients by the NIWA Water Quality Laboratory (Hamilton, NZ). Total Organic Carbon (TOC) was determined via high temperature catalytic oxidation followed by infrared detection (Clesceri et al. 1998). Dissolved reactive phosphorus (DRP), ammonium, and total nitrogen (nitrate + nitrite) were determined using an Astoria Analyser (Astoria-Pacific) according to EPA standard methods (EPA 1983). Nitrite alone was measured according to (Lachat Instruments 2008) standard procedures. Samples were diazotized with sulphanilamide followed by the addition of $\mathrm{N}$-(1-napthyl)-ethylenediamine dihydrochloride and colorometric detection.

Seawater chlorophyll concentration in these seawater samples was determined according to methods modified from (Wasmund et al. 2006). For each sample, $0.5-4 \mathrm{~L}$ of seawater was filtered through a $47 \mathrm{~mm}$ diameter cellulose acetate (CA) filter with a $0.22 \mu \mathrm{m}$ pore size (Membrane Solutions, USA). A Rocker 400 oil-free vacuum pump was used to provide suction (Rocker Scientific, Taiwan). Samples were then stored in the dark at $20^{\circ} \mathrm{C}$ prior to extraction. Samples were extracted using $95 \%$ ethanol for $24 \mathrm{~h}$ in the dark at $4^{\circ} \mathrm{C}$ followed by centrifugation at $16,100 \mathrm{x}$ g for $10 \mathrm{~min}$ to clear the sample of cellular debris. An aliquot $(250 \mu \mathrm{l})$ of each sample was loaded into each well of a clear polystyrene 96 well microplate (Greiner Bio-One, Germany), giving a path-length of $0.759 \mathrm{~mm}$. Absorbance was read at 632, 649, 665, 696 and $750 \mathrm{~nm}$ using an Enspire 2300 spectrophotometer (Perkin Elmer, USA) set to default scanning settings. These readings were then entered into the quadrichroic equations specified in Ritchie (2008), in order to calculate the concentration of total chlorophyll and chlorophyll $a$. 


\subsection{Laboratory component}

\subsubsection{Experimental design}

During March 2014, 300 specimens of Anthopleura aureoradiata were collected from Pauatahanui Inlet, Wellington ( $41^{\circ} 06^{\prime} 005^{\prime}$ 'S , 174 $\left.52^{\prime} 017^{\prime \prime} \mathrm{E}\right)$. Anemones were collected from this site due to the limited availability of anemones at the seasonal comparison site (Point Halswell). The anemones were transported back to the lab in fresh seawater collected on site, detached from their host cockle shells, and transferred to $500 \mathrm{ml}$ glass beakers at a density of 20 anemones per beaker. Five beakers were distributed into each of three independent water baths, with the temperature being controlled by HAAKE C10 water heaters. Each beaker contained $350 \mathrm{ml}$ of $20 \mu \mathrm{m}$ filtered seawater (FSW) and was maintained at $15 \pm 1{ }^{\circ} \mathrm{C}$ under a $12 \mathrm{~h} \mathrm{light/dark} \mathrm{cycle.} \mathrm{Irradiance} \mathrm{was} \mathrm{maintained} \mathrm{at} 150 \pm$ $10 \mu \mathrm{mol}$ photons $\mathrm{m}^{-2} \mathrm{~s}^{-1}$ as measured incident on the water surface of each beaker using a LI-COR LI-250A light meter. Anemones were fed Artemia sp. nauplii three times per week and the beakers cleaned, including a complete transfer to fresh FSW one day following feeding. Anemones were maintained under these conditions to acclimatise to control experimental conditions for two months prior to the initiation of the experiment.

Treatments were set up as 'cold' $\left(8 \pm 1^{\circ} \mathrm{C}\right)$, ambient (control) $\left(15 \pm 1^{\circ} \mathrm{C}\right)$, and 'hot' $(25 \pm$ $1^{\circ} \mathrm{C}$ ), with each treatment containing five replicates (Figure 2.1) Temperatures were chosen to replicate field conditions based on historical climate data (National Institute of Water and Atmospheric Research 2015). Temperatures were ramped from control to treatment levels in even increments over a six-day period and then maintained at treatment levels by HAAKE C10 water heaters. 

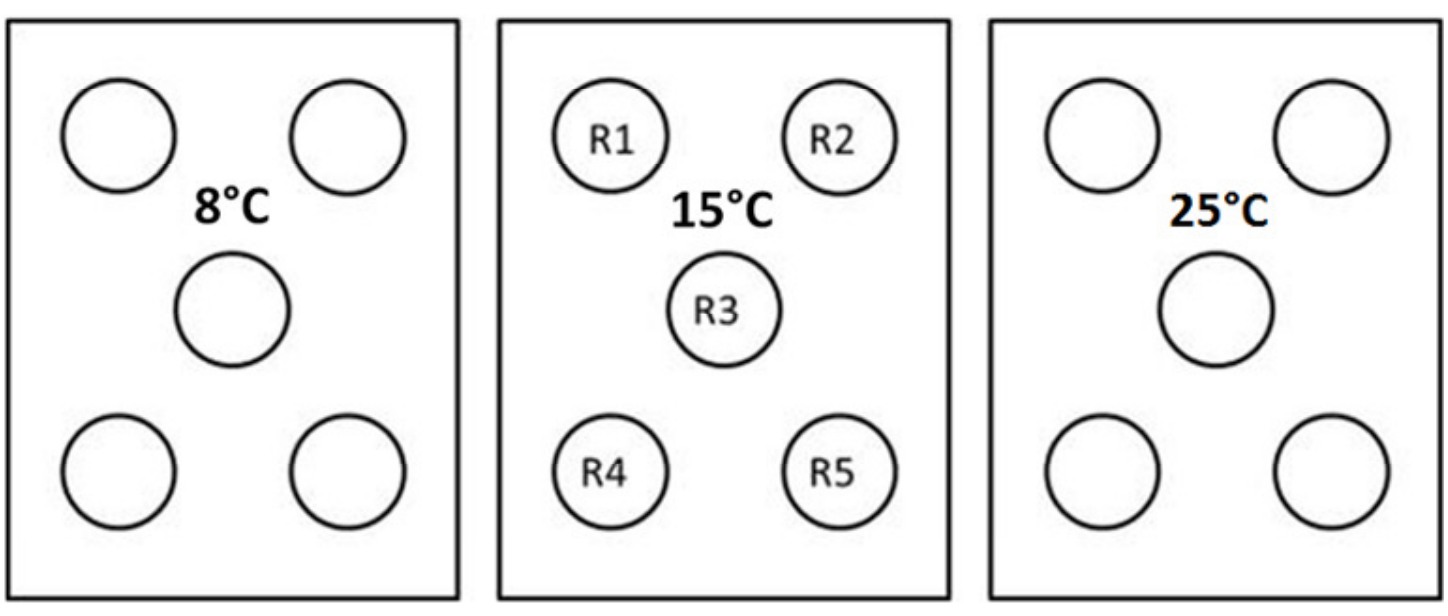

Figure 2.1. Experimental set up. Rectangles denote water baths, circles denote beakers. Each treatment contained five biological replicates. The temperature values represent the temperatures achieved following acclimation and ramping to experimental conditions for cold, ambient and hot treatments, respectively.

Anemones were sampled on two dates, once prior to the temperature being increased and once following six weeks of treatment. Sampling was conducted during the middle of the light cycle to ensure that a metabolite profile containing photosynthesis light-cycle compounds was taken. Sampling also followed a five day starvation period to ensure that no Artemia sp. nauplii remained in the anemone's digestive tract, which would contaminate the samples and alter the resulting metabolite profile. For each replicate, four anemones were taken at random from each beaker. These were rinsed in FSW, placed in a $2 \mathrm{ml}$ cryovial then spun for 10 seconds at 2,000 x g. The remaining water was removed and the cryovial placed directly into $\mathrm{LN}_{2}$. The entire sampling process took less than one minute per replicate. Samples were then transferred to a $-80^{\circ} \mathrm{C}$ freezer and stored ready for analysis. Upon removal from the freezer, samples were first rinsed in cold $\left(4^{\circ} \mathrm{C}\right)$ MilliQ water. Cold MilliQ water $(600 \mu \mathrm{l})$ was then added to each sample and they were homogenised for 1 min using an IKA T10 BS5 motorised homogeniser (IKA, USA). The homogeniser was rinsed twice with methanol and then with cold MilliQ water between samples. Cold MilliQ ( $1 \mathrm{ml}$ ) was then added to each sample. A $150 \mu \mathrm{l}$ aliquot was taken for the determination of host protein and chlorophyll $a$ and $c_{2}$ content. A final $100 \mu \mathrm{l}$ aliquot was taken in order to determine Symbiodinium density. The remaining homogenate was used for GC-MS analysis. 


\subsubsection{Host protein content}

Protein content was determined according to the methods of Bradford (1976) with the linearization method of Zor \& Selinger (1996) applied. Samples were centrifuged at $16,100 \mathrm{x} \mathrm{g}$ for $20 \mathrm{~min}$ to isolate proteins. A $10 \mu \mathrm{l}$ aliquot of each sample was then placed into the well of a 96 well-plate. Ten technical replicates were run per sample. A standard curve ranging from $0-1 \mathrm{mg} / \mathrm{ml}$ protein was set up in each plate using bovine serum albumin (BSA). Bradford reagent $(250 \mu \mathrm{l})$ was then added to each well and the plate was incubated in the dark for five minutes. The absorbance of each well was read at $595 \mathrm{~nm}$ and $450 \mathrm{~nm}$ wavelengths using an Enspire 2300 microplate reader (Perkin-Elmer, USA). The unknown protein concentrations of each sample were then calculated against the BSA standard curve.

\subsubsection{Symbiodinium density}

Symbiodinium cell density was determined by counting using an Improved Neubauer haemocytometer (Boeco, Germany). The samples were first diluted 10-20 times in order to enable accurate counting. Each sample was vortexed immediately prior to counting. A glass Pasteur pipette was then used to introduce the sample to the counting chamber. Finally, the haemocytometer was viewed under $10 \mathrm{x}$ magnification and the number of Symbiodinium cells recorded. Six to twelve replicate counts were taken per sample. The average of these counts was normalised against host protein content to provide values in cells/mg protein.

\subsubsection{Symbiodinium chlorophyll a \& $c_{2}$ content}

The chlorophyll $a$ and $c_{2}$ content of Symbiodinium cells isolated from host anemones was determined as a measure of symbiont photosynthetic potential (Emerson 1929). Anemone homogenate was spun at $16,100 \mathrm{x}$ g for $10 \mathrm{~min}$ to isolate Symbiodinium cells. A $300 \mu \mathrm{l}$ aliquot of $\mathrm{ddH}_{2} \mathrm{O}$ was then added to the pellet and this preparation milled at $50 \mathrm{~Hz}$ for 2 min in a chilled Qiagen TissueLyser LT (Qiagen, Netherlands) to re-suspend the pellet. Next, $2700 \mu \mathrm{l}$ of cold HPLC-grade acetone were added to each sample and these were stored in the dark at $4^{\circ} \mathrm{C}$ for $24 \mathrm{~h}$. The solution was then spun at $16,100 \mathrm{x} g$ for $5 \mathrm{~min}$ to clear the sample of cellular debris. Triplicate readings of the absorbance of each sample were then taken at 630, 664 and $750 \mathrm{~nm}$ wavelengths using a Shimadzu UV2550 
spectrophotometer (Shimadzu, Japan). Finally, simultaneous equations were used to calculate the concentration of chlorophyll $a$ and $c_{2}$ in $\mu \mathrm{g} / \mathrm{ml}$ (Ritchie 2006). These readings were normalised to Symbiodinium cell density and scaled to provide values in $\mathrm{pg} / \mathrm{cell}$.

\section{$2.3 \quad$ GC-MS protocol}

Sample preparation for GC-MS was based on methods modified from Roessner et al. (2000), Villas-Bôas et al. (2006) and Burriesci et al. (2012).

\subsubsection{Host-symbiont separation}

Samples were kept on ice at all times during processing. Samples were first centrifuged at $2000 \mathrm{x} \mathrm{g}$ for $5 \mathrm{~min}$ at $4^{\circ} \mathrm{C}$ and the supernatant containing host material transferred to a new $2 \mathrm{ml}$ microfuge tube. The remaining algal pellet was washed with cold milliQ water, re-spun and the supernatant discarded. The host fraction was also re-spun and the supernatant collected multiple times until no algal pellet remained. Both cleaned fractions were placed in $\mathrm{a}-80^{\circ} \mathrm{C}$ freezer for $30 \mathrm{~min}$ and then placed in a Labconco 73820 freezedryer (Labconco, USA) and run on the automatic setting overnight.

\subsubsection{Metabolite extraction}

On removal from the freeze dryer, $1.5 \mathrm{ml}$ of cold $50 \%$ methanol were added to both host and symbiont fractions, followed by $5 \mu \mathrm{l}$ of $10 \mathrm{mM}$ ribitol as an internal standard. (against which to calculate the relative concentrations of metabolites). Samples were mixed vigorously using a vortex mixer for $1 \mathrm{~min}$; symbiont samples were pipette mixed if vortexing was insufficient. The host samples were then vortexed for $2 \mathrm{~min}$, centrifuged at $2000 \mathrm{x} g$ for $6 \mathrm{~min}$ at $-9^{\circ} \mathrm{C}$, and the supernatant collected in a $15 \mathrm{ml}$ Falcon ${ }^{\circledR}$ tube. A 1.5 $\mathrm{ml}$ aliquot of cold $80 \%$ methanol was then added to the initial host microfuge tube and it was vortexed for $2 \mathrm{~min}$ and then centrifuged at $2000 \mathrm{x}$ g for $15 \mathrm{~min}$ at $-9^{\circ} \mathrm{C}$. Both host extracts were then pooled in the $15 \mathrm{ml}$ tube and kept on dry ice.

To the symbiont samples, approximately ten methanol-chloroform washed glass beads were added followed by milling for $6 \mathrm{~min}$ at $50 \mathrm{~Hz}$ in a chilled Qiagen TissueLyser LT (Qiagen, Netherlands). The samples were then centrifuged at $2000 \mathrm{x}$ g for 6 min at $-9^{\circ} \mathrm{C}$ 
and the supernatant collected in a $15 \mathrm{ml}$ Falcon tube. The remaining algal pellet was extracted again using $1.5 \mathrm{ml}$ of cold $80 \%$ methanol, followed by further centrifugation at $2000 \mathrm{x} g$ for $15 \mathrm{~min}$ at $-9^{\circ} \mathrm{C}$. This supernatant was then pooled with the first symbiont extraction in the $15 \mathrm{ml}$ tube and kept on dry ice.

To both host and symbiont extracts, $9 \mathrm{ml}$ of cold milliQ water were then added, giving a methanol dilution of $<20 \%$ to facilitate freezing. Next, three sugar standard mixes were constructed, each containing a particular set of sugars at a concentration of $10 \mathrm{mM}$. Mix one contained ribose, glucose and fructose. Mix two contained arabinose, galactose, mannitol, glucose and ribitol. Mix three contained xylose, ribitol, mannose and sucrose. All samples and sugar mixes were then placed in the $-80^{\circ} \mathrm{C}$ freezer for $30 \mathrm{~min}$ followed by overnight freeze-drying. In order to concentrate the samples, on removal from the freeze-dryer $200 \mu \mathrm{l}$ of cold milliQ water were used to thoroughly wash out the $15 \mathrm{ml}$ tubes. This solution was then transferred to $2 \mathrm{ml}$ microfuge tubes and frozen at $-80^{\circ} \mathrm{C}$ for $30 \mathrm{~min}$, placed in the freeze-dryer overnight, and then placed in a desiccator for another night to ensure that the samples were thoroughly dry.

\subsubsection{TMS derivitisation}

Only ten samples at a time were derivatised and run on the GC-MS, as the chemicals used are very unstable and break down in the time it takes to run samples through the machine. An $80 \mu \mathrm{l}$ aliquot of methoxyamine hydrochloride in pyridine $(20 \mathrm{~g} / \mathrm{l})$ was added to each sample and the sugar mixes. These were sealed, vortexed for a minimum of one minute, transferred to GC-MS vial inserts, sealed again, then incubated at $30^{\circ} \mathrm{C}$ for $30 \mathrm{~min}$. Following this, $80 \mu \mathrm{l}$ of $\mathrm{N}$-methyl-N-(trimethylsilyl trifluoroacetamide) (MSTFA) were added and the samples pipette-mixed three times. These were incubated a final incubation at $37^{\circ} \mathrm{C}$ for $90 \mathrm{~min}$ and run on the GC-MS within $2 \mathrm{~h}$.

\subsubsection{GC-MS analysis}

In order to identify and quantify metabolites, the derivatised samples were run through a Shimadzu Gas Chromatograph Mass Spectrometer QP2010 Plus equipped with a quadrupole mass selective detector (Shimadzu, Japan). GC-MS settings were based on (Villas-Bôas et al. 2006). One microlitre of each sample was injected using an AOC-20i+s auto-sampler into the inlet, which was fitted with a Restek single taper gooseneck "Sky" 
liner packed with deactivated glass wool (split/splitless capable) with dimensions of: 3.5 $\mathrm{mm}$ ID x $5.0 \mathrm{~mm}$ OD x $95 \mathrm{~mm}$. The inlet temperature was maintained at 230C and a 20:1 split ratio applied. Column flow was set to $1 \mathrm{ml} / \mathrm{min}$ constant flow mode and purge flow at $18 \mathrm{ml} / \mathrm{min}$. The column used was a $30 \mathrm{~m}$ fused silica RTX-1701 with a $0.25 \mathrm{~mm}$ ID and

film thickness of $0.25 \mu \mathrm{m}$. The stationary phase was cross-bonded $14 \%$ cyanopropylphenyl, 86\% dimethylpolysiloxane, Carrier gas was instrument grade helium (BOC). GC oven temperature was initially set at $70^{\circ} \mathrm{C}$ and held for $5 \mathrm{~min}$. The temperature was then increased at $10^{\circ} \mathrm{C} / \mathrm{min}$ to $179^{\circ} \mathrm{C}$ and then at $0 .{ }^{50 \mathrm{C}} / \mathrm{min}$ to $180^{\circ} \mathrm{C}$, where it was held for $2 \mathrm{~min}$. It was then increased at $10^{\circ} \mathrm{C} / \mathrm{min}$ to $220^{\circ} \mathrm{C}$ and held for 1 min. Following this, the temperature was increased at $2.5^{\circ} \mathrm{C} / \mathrm{min}$ to $265^{\circ} \mathrm{C}$, held for $1 \mathrm{~min}$, and then increased at $10^{\circ} \mathrm{C} / \mathrm{min}$ until it reached $280^{\circ} \mathrm{C}$, where it was held for a final $1 \mathrm{~min}$. The interface between the gas chromatograph and the mass spectrometer was maintained at $280^{\circ} \mathrm{C}$, and the ion source at $230^{\circ} \mathrm{C}$. Ionisation was via positive-ion electron-impact ionisation at $70 \mathrm{eV}$. The mass analyser was run in scan mode, scanning from $50-900$ amu at a rate of $2000 \mathrm{amu} / \mathrm{sec}$. The detector was turned on $7.5 \mathrm{~min}$ into the run.

\subsection{Data processing \& analysis}

IBM SPSS Statistics v22 was used to run all statistical tests regarding seasonal variation in environmental conditions at the field site. It was also used to run all statistical tests regarding Symbiodinium cell density and chlorophyll $a$ and $c_{2}$ content in both field and laboratory samples. Two-way ANOVA (without replication) was conducted to determine whether any difference existed between seasons (winter vs. summer) with respect to the maximum daily gust, wind direction, daily rainfall, daily maximum and minimum temperature, daily radiation intensity, or seawater TOC, DRP, ammonium, total nitrogen, nitrite and chlorophyll concentrations. Two-way ANOVA (without replication) was also conducted to determine whether any difference existed in Symbiodinium cell density or chlorophyll $a$ and $c_{2}$ content between anemones collected in different seasons (winter $v s$. summer).

For the laboratory experiment, one-way ANOVA was used to determine whether Symbiodinium cell density or chlorophyll $a$ and $c_{2}$ content varied: (1) between treatment groups (cold, ambient or hot) immediately prior to the initiation of treatment; (2) prior to and following six weeks of treatment in the control; and (3) between hot and ambient, 
cold and ambient, and hot and cold treatments, following six weeks of treatment. Tukey's post hoc tests were performed as appropriate following all one-way ANOVAs. All data was checked for normality and homogeneity of variances prior to performing tests and transformed to improve normality. Where normality could not be improved raw data was used for all statistical analyses. Parametric tests were still used for these cases as ANOVA is generally robust to deviations from normality. However, when data had unequal variances Welch's ANOVA or the SPSS unequal variance adjusted t-test were used instead.

Metabolomic data processing was based on (Smart et al. 2010). First, a subset of the NIST 08 library containing a list of all possible compounds in the analysed samples was generated. To do this, peak identification of the raw GC-MS data for each sample (chromatograms/spectra) was conducted against the NIST 08 library in Chemstation. The lists of identified compounds were then compiled using the $\mathrm{R}$ package 'Metab' developed by Aggio et al. (2011). This compound list was then used to construct a library in the MSP file format using Lib2NIST converter. Finally, the MSP library was converted to MSL format using AMDIS (the Automated Mass spectral Deconvolution and Identification System). Next, peak identification was conducted in AMDIS against the newly constructed NIST subset library and a batch report of the results was generated. AMDIS was used for peak identification as it provides superior peak deconvolution. Finally, using the AMDIS batch report as a reference, the chromatograms of each sample were integrated in $\mathrm{R}$ using the Metab package. The final output of this process was a file containing information on the metabolites identified in each sample and the peak area of the reference ion of each of those metabolites. The peak area values were then normalised to dry weight biomass and the internal standard ribitol to determine the relative quantity of each metabolite.

Compound names were converted from their silylated form to their pre-silylated form based on the common silylation reactions as described by Villas-bôas et al. (2011) (Figure 2.2). These final compound names were confirmed against a TMS library preconstructed by The Metabolomics Lab at the University of Auckland. 
$\widehat{\mathrm{R}} \widehat{\mathrm{NH}_{2}}$<smiles>[R]C(=O)O</smiles>

$\widehat{\mathrm{R}} \widehat{\mathrm{OH}}$<smiles>CN(C(=O)C(F)(F)F)[Si](C)(C)C</smiles>

MSTFA

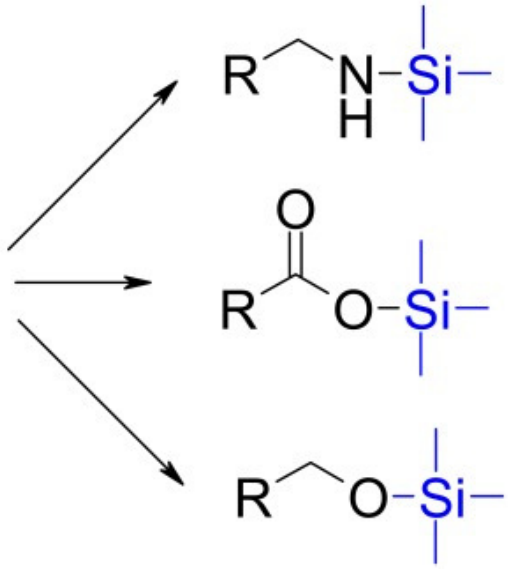

Figure 2.2 Common MSTFA silylation reactions (Villas-bôas et al. 2011) Licenced under CC BY 3.0 https://creativecommons.org/licenses/by/3.0/legalcode

The online metabolomics analysis tool suite, MetaboAnalyst (Xia et al. 2012; Xia et al. 2015; Xia \& Wishart 2011) was used to explore the metabolomics data via univariate and multivariate analysis. Raw metabolomics data was transformed using the generalised logarithm transformation (glog) and Pareto scaled prior to statistical analysis to improve their biological relevance (van den Berg et al. 2006). This process removes the dependency of statistical analysis on the average concentration of metabolites and the magnitude of fold changes seen between groups. This is important, as a relatively small change in a highly abundant metabolite is not necessarily more important (in a biological sense) than a relatively large change in a low concentration metabolite, even though the total change in concentration would be a lot greater. Also, a large fluctuation in a metabolite that naturally undergoes large fluctuations is not necessarily more important than a small fluctuation in the concentration of a metabolite that naturally has a very stable concentration. All analyses were conducted across the following combination of groups: Field samples collected in the winter versus summer; between the cold, ambient $\&$ hot experimental treatments immediately prior to the initiation of treatment; in the ambient experimental treatment prior to and following six weeks of treatment; and between hot and ambient, cold and ambient, and hot and cold experimental treatments following six weeks at treatment temperatures. Fold change analysis was conducted prior to the normalisation of data. All fold changes equivalent to $a \geq 2$ fold increase (i.e. $\geq 2$ in metabolites that displayed an increase in concentration and $\leq 0.5$ in metabolites whose 
concentration decreased) are reported. Next, one-way ANOVA, followed by Tukey post hoc tests (as appropriate) were performed to determine whether any differences existed in the level of individual metabolites between groups. Next, principle components analysis (PCA) was performed to determine whether any differences in metabolite profile existed between groups from a multivariate perspective, and to identify the contribution of each metabolite to any observed shifts in metabolite profile. The metabolite profiles are visualised via $2 \mathrm{D}$ principal component plots, which compare control to treatment groups. The contribution of each metabolite to the principal component scores of each group is summarised in the corresponding loadings plots.

Finally, the pathway activity profiling (PAPi) package for R (Aggio et al. 2010) was used to determine the biochemical pathways identified in each sample based on the input list of metabolites and to generate a pathway activity score (AS) for each identified pathway in every sample. The generation of a pathway AS is based on the assumption that when a pathway is highly active, more metabolites from that pathway will be identified, but the concentration of these metabolites will be lower due to increased flux through the pathway, and vice versa when pathway activity is low (Aggio et al. 2010). The pathways identified by PAPi come from an indiscriminate search of the KEGG database (KEGG, Kyoto). This database contains information from a wide range of organisms and metabolic pathways representing overall metabolic processes such as 'metabolic pathways', hence numerous pathways that do not exist in the study species or that were irrelevant to the study system were identified (Booth et al. 2013). To account for this, the list of metabolic pathways identified by PAPi were manually filtered to remove irrelevant pathways prior to further analysis, as suggested by Booth et al. (2013). IBM SPSS Statistics v22 was then used to check pathway AS's in all treatment groups for normality, via the Shapiro-Wilk test, and homogeneity of variances, via the Levene's test for equality of variances (either parametric or non-parametric as appropriate (Nordstokke \& Zumbo 2010; Nordstokke et al. 2011)). Independent samples t-tests were then run to determine whether there was any difference in the mean AS between treatment groups for each of the identified pathways. An adjusted t-test, which does not assume equal variances, was run for all samples that failed the Levene's test for equality of variances. The output of this test is provided, as appropriate, in place of the standard t-test results. As a pathway's AS is "inversely related to the predicted pathway activity" (Aggio et al. 2010), inverted and $\log$ transformed AS's were plotted to provide an intuitive graphical representation of 
the differences in predicted pathway activity. Furthermore, the data was scaled to set the AS for each pathway in the control group to zero, enabling visualisation of the change in predicted pathway activity in the treatment relative to the control group (Aggio et al. 2010). 


\section{Chapter 3: $\quad$ Results}

This chapter is presented as two separate components: a field component and a laboratory component. Both the field and laboratory components are further separated according to what partner from the symbiosis is under question: the symbiont (Symbiodinium sp.) or the host (Anthopleura aureoradiata). Within the laboratory component results are presented according to treatment, being either cold or hot.

Environmental conditions were measured in the field for two weeks prior to each collection date. Indicators of the state of the symbiotic relationship were also measured for all field and laboratory samples. In order to characterise the metabolite profiles and to determine the impact of environmental stressors on metabolic pathway activity in both host and symbiont, metabolites were identified via GC-MS analysis. A total of 311 metabolites were identified in host anemones and 65 metabolites were identified in the symbionts. By applying pathway activity profiling (PAPi) to these identified metabolites, 113 metabolic pathways were identified in the host anemones and 71 pathways were identified in the symbionts.

\subsection{Exploring seasonal trends in the metabolic functioning of $A$. aureoradiata and their symbionts}

\subsubsection{Seasonal environmental conditions}

\subsubsection{Weather}

Maximum daily temperature (Tmax) and minimum daily temperature (Tmin) were the only measured weather conditions that differed significantly between seasons at the field site. Tmax was $5.6^{\circ} \mathrm{C}$ lower in winter than summer $\left(\mathrm{t}_{19}=-6.638, \mathrm{p}<0.001\right)$. Tmin was $7.4^{\circ} \mathrm{C}$ lower in winter than summer $\left(\mathrm{t}_{17}=-6.330, \mathrm{p}<0.001\right)$. Daily rainfall and radiation levels remained relatively homogenous between seasons (Figure 3.1). 


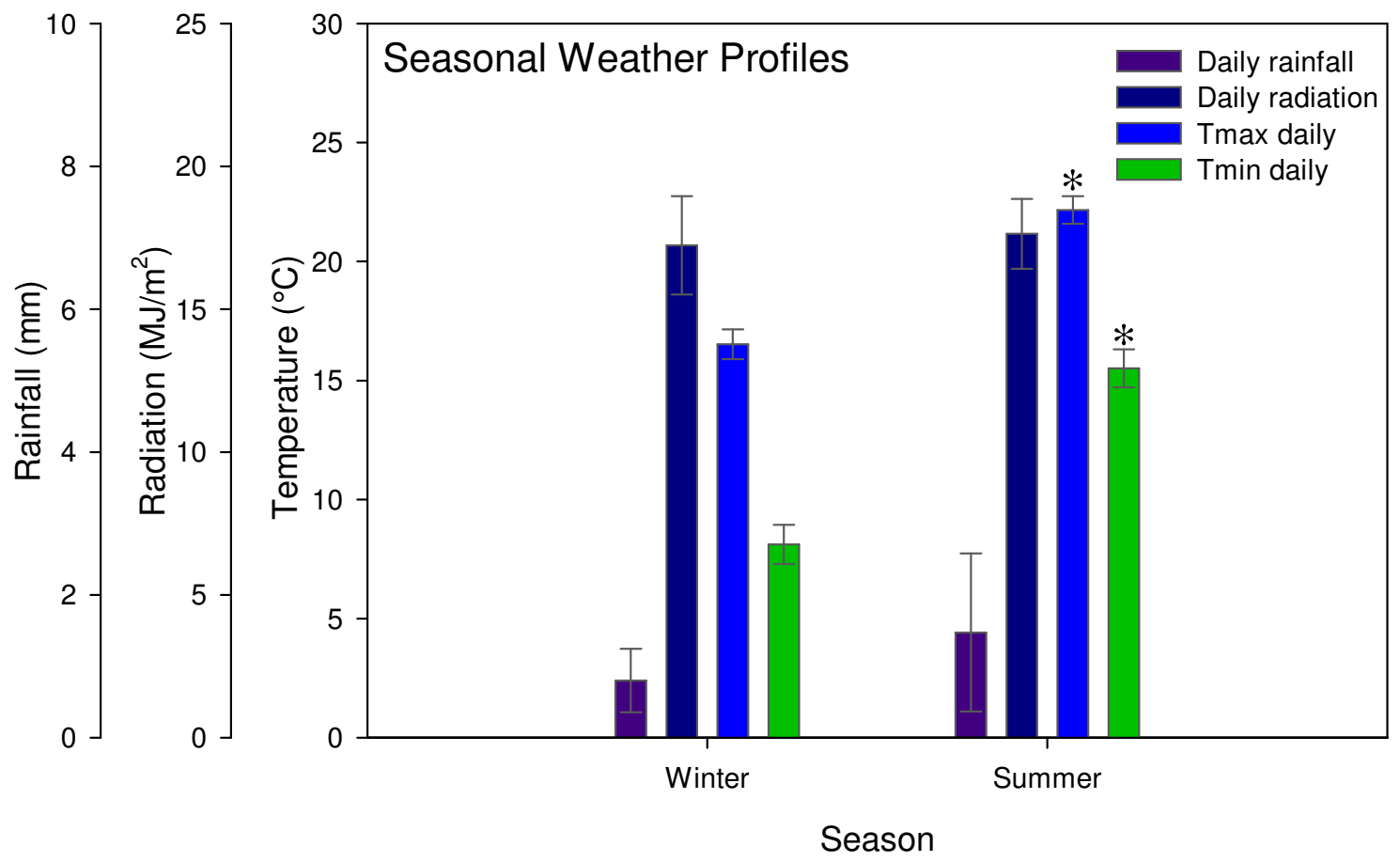

Figure 3.1 Weather conditions recorded daily at the Wellington Kelburn automatic weather station (AWS) over a two week period prior to anemone collection in winter 2014 and summer 2015. The recorded parameters include daily rainfall, average daily radiation, maximum daily temperature (Tmax) and minimum daily temperature (Tmin). Values are means $\pm \mathrm{SE}($ Summer $v s$. winter, t-test, $* P<0.001)$

\subsubsection{Nutrients}

Total organic carbon (TOC), nitrate $\left(\mathrm{NO}_{3}-\mathrm{N}\right)$, chlorophyll $a(\mathrm{Chl} a)$ and total chlorophyll (Total Chl) all differed significantly between seasons. The TOC concentration was 4072.5 $\mathrm{mg} / \mathrm{m}^{-3}$ greater in summer than winter $\left(\mathrm{t}_{6}=-3.605, \mathrm{p}<0.05\right)$. The $\mathrm{NO}_{3}-\mathrm{N}$ concentration was $5.5 \mathrm{mg} / \mathrm{m}^{-3}$ greater in winter than summer $\left(\mathrm{t}_{6}=3.740, \mathrm{p}<0.05\right)$. The Chl a concentration was $1.2 \mathrm{mg} / \mathrm{m}^{-3}$ greater in summer than winter $\left(\mathrm{t}_{6}=-3.283, \mathrm{p}<0.05\right)$. The Total Chl concentration was $3.1 \mathrm{mg} / \mathrm{m}^{-3}$ greater in summer than winter $\left(\mathrm{t}_{6}=3.740, \mathrm{p}<\right.$ 0.05). Dissolved reactive phosphorus (DRP) and ammonium $\left(\mathrm{NH}_{4}-\mathrm{N}\right)$ did not differ significantly between seasons (Figure 3.2). 


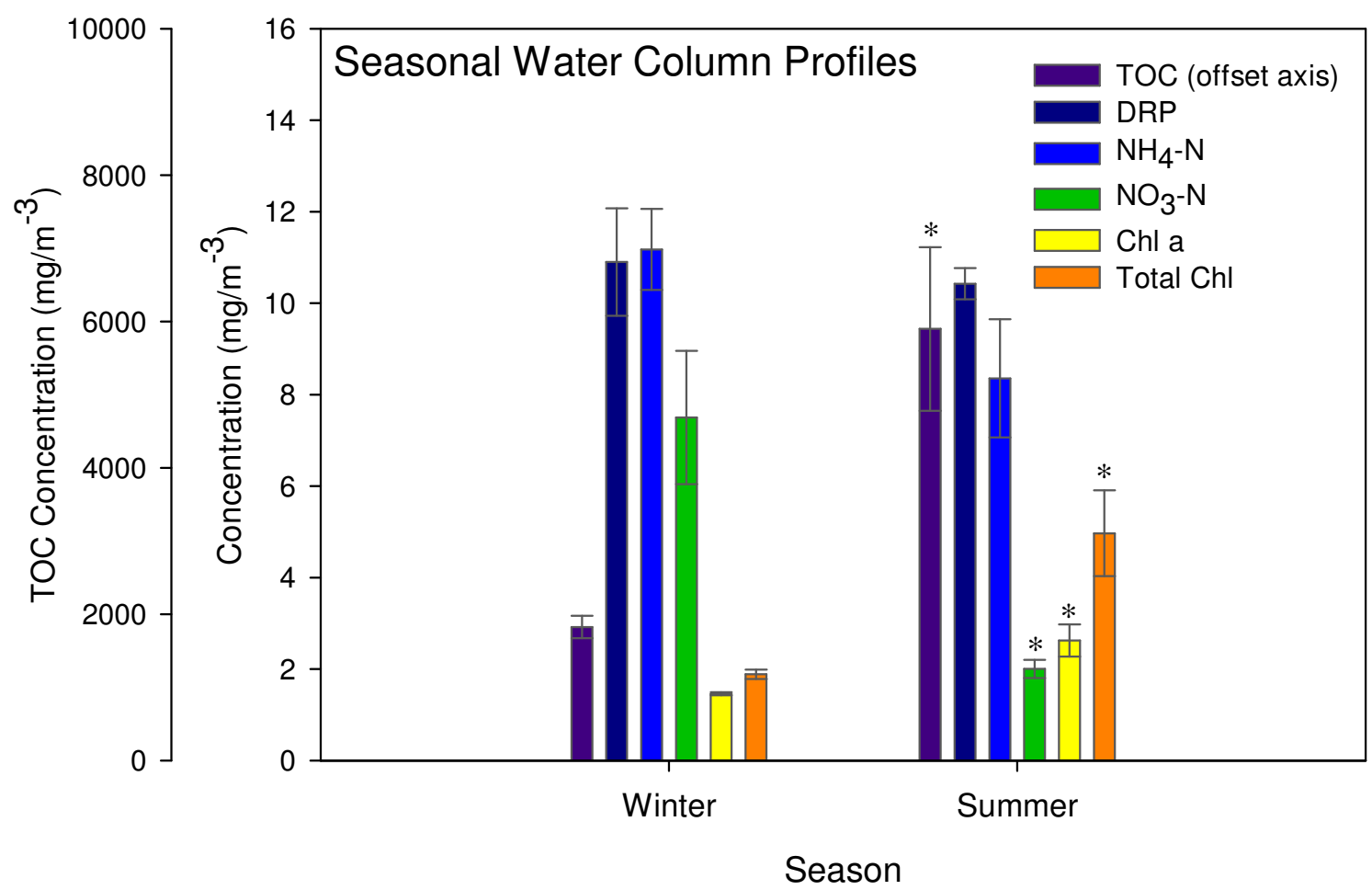

Figure 3.2: Total organic carbon (TOC), dissolved reactive phosphorus (DRP), ammonium $\left(\mathrm{NH}_{4}-\mathrm{N}\right)$, nitrate $\left(\mathrm{NO}_{3}-\mathrm{N}\right)$, chlorophyll $a(\mathrm{Chl} a)$ and total chlorophyll (Total Chl) concentrations in surface seawater at the field collection site in winter 2014 and summer 2015. Values are means \pm SE. (Summer $v s$. winter, t-test, $* P<0.05$ ).

\subsubsection{State of the symbiosis between seasons}

No significant difference was detected in Symbiodinium cell density, or chlorophyll $a$ and chlorophyll $c_{2}$ concentrations per symbiont cell between anemones sampled in summer versus those sampled in winter. However, there was a trend towards higher concentrations of chlorophyll $a$ and lower concentrations of chlorophyll $c_{2}$ per symbiont cell in anemones sampled in summer as opposed to those sampled in winter (Figure 3.3). 


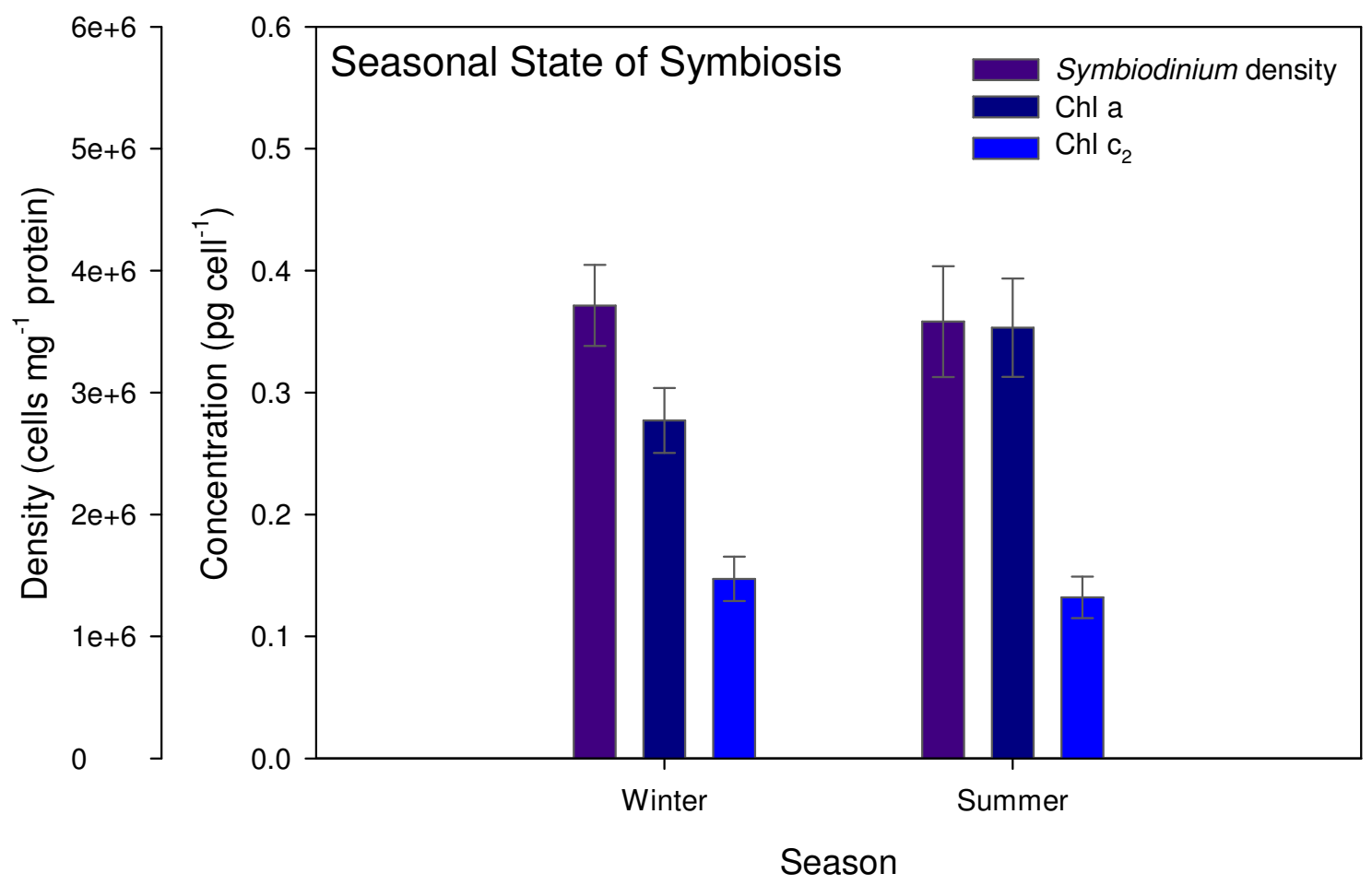

Figure 3.3: Symbiodinium cell density, and chlorophyll $a$ and $c_{2}$ concentrations per symbiont cell in anemones sampled in winter 2014 and summer 2015. Values are means \pm SE. (Summer v winter, t-test, $* P<0.05)$.

\subsubsection{Comparison of metabolite profiles between Seasons}

\subsubsection{Symbiont}

\subsection{Comparison of individual metabolites}

Figure 3.4 shows the top 25 metabolites based on p-values (t-test, $n=5$ independent samples) when comparing the metabolite profile of symbionts from anemones sampled in summer versus those sampled in winter. Of these 25 metabolites, seven showed a significant difference at the $\mathrm{p}<0.05$ level. These compounds included the sugar: mannitol; the glycoside: methyl galactoside; the parent compound of the amphetamine group: amphetamine (FC, 0.41892); the fatty acid: cis-5,8,11,14,17-eicosapentaenoic acid; the alcohol: isoprenyl alcohol; the monoterpene: 1-isopropenyl-2,3dimethylcyclopentane and the unclassified compound: 11,11-dimethyl-spiro[2,9]dodeca3,7-dien. 


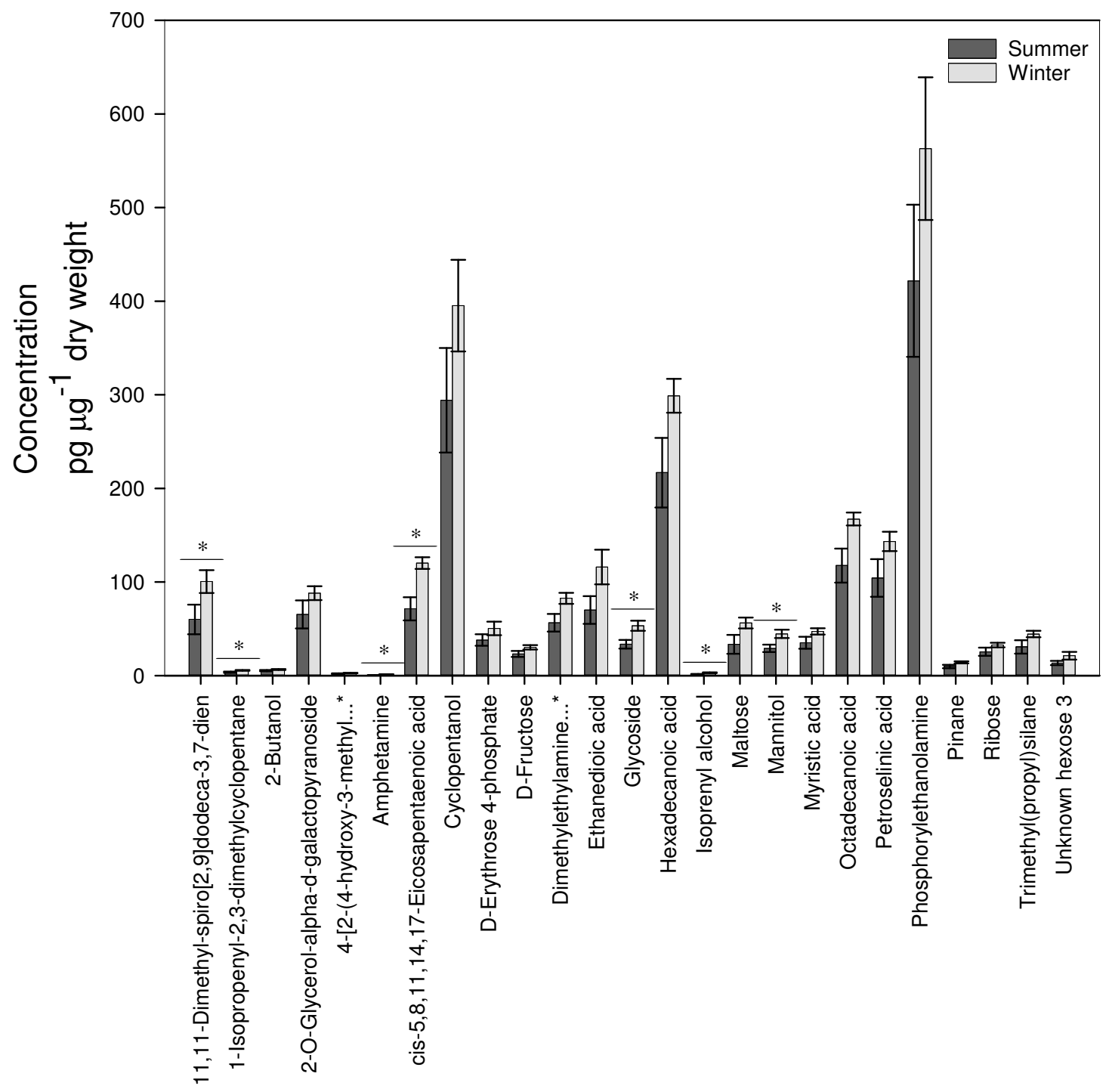

Figure 3.4: Concentration of individual metabolites per $\mu \mathrm{g}$ dry weight in Symbiodinium $s p$. isolated from A. aureoradiata sampled in either summer or winter. Values are means $( \pm \mathrm{SE})$ and asterisks indicate significant differences between summer and winter (t-test, $\mathrm{n}$ $=5, * \mathrm{p}<0.05)$. (Concatenated names marked $(. . *)$, see appendix for full details).

\subsection{Multivariate analysis (PCA)}

From a multivariate perspective there was no clear difference in the metabolite profile of symbionts from anemones sampled in summer versus winter (Figure 3.5). Both the 
summer and winter groups overlap to a large degree on the 2D PCA scores plot, with PC1 explaining $54.7 \%$ of the observed variance and PC2 16.3\%. However, there is much greater within-group variability in the summer samples compared to the winter samples. The corresponding loadings plot also shows a significant spread in the metabolites, indicative of the high degree of within-group variability in the summer samples. The metabolites with the highest absolute loadings scores are the most influential in driving the observed variance. On the loadings 1 axis these metabolites included the amino acid: ornithine; the sugar: maltose and the amine: 1,4-butanediamine. On the loadings 2 axis it included the amino acid derivative: alanine, N-methyl-n-butoxycarbonyl-, isohexyl ester; the sugar: beta-D-galactofuranose; the glycoside: methyl beta-D-glucopyranoside; and the acid: methanesulfinic acid.
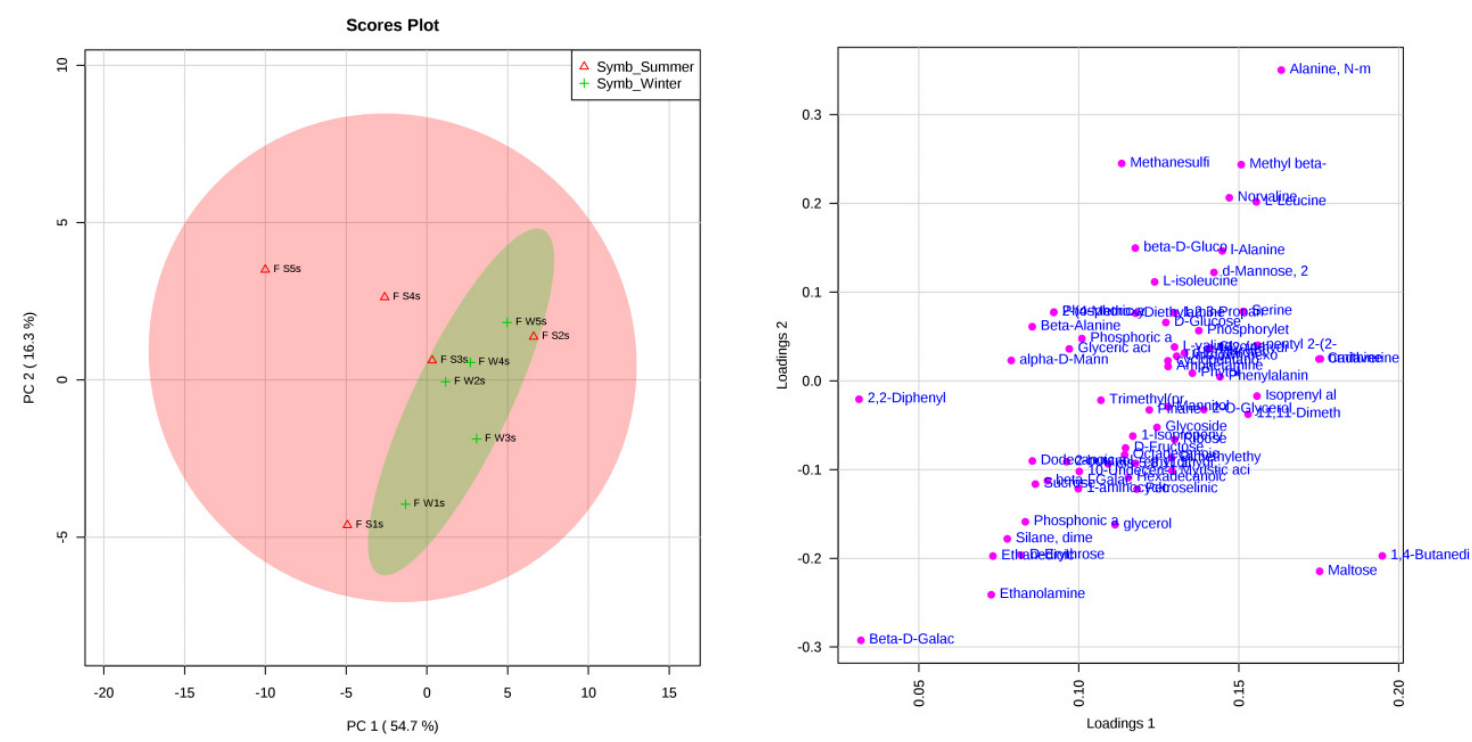

Figure 3.5: PCA scores plot with shaded $95 \%$ confidence intervals (left) and corresponding loadings plot (right) of symbionts isolated from anemones collected in summer compared to those collected in winter, providing a multivariate perspective on their metabolite profiles.

\subsection{Metabolic pathway response}

Most of the detected metabolic pathways showed a trend towards increased activity in symbionts isolated from anemones sampled in summer relative to those sampled in winter. The 20 metabolic pathways expressing the largest absolute change in summer 
versus winter (Figure 3.6) were made up of seven pathways involved in lipid metabolism, six in carbohydrate metabolism, two in the biosynthesis of secondary metabolites, one in the metabolism of co-factors and vitamins, one in cell growth and death, one in the digestive system, one in membrane transport and one in environmental information processing-signal transduction.

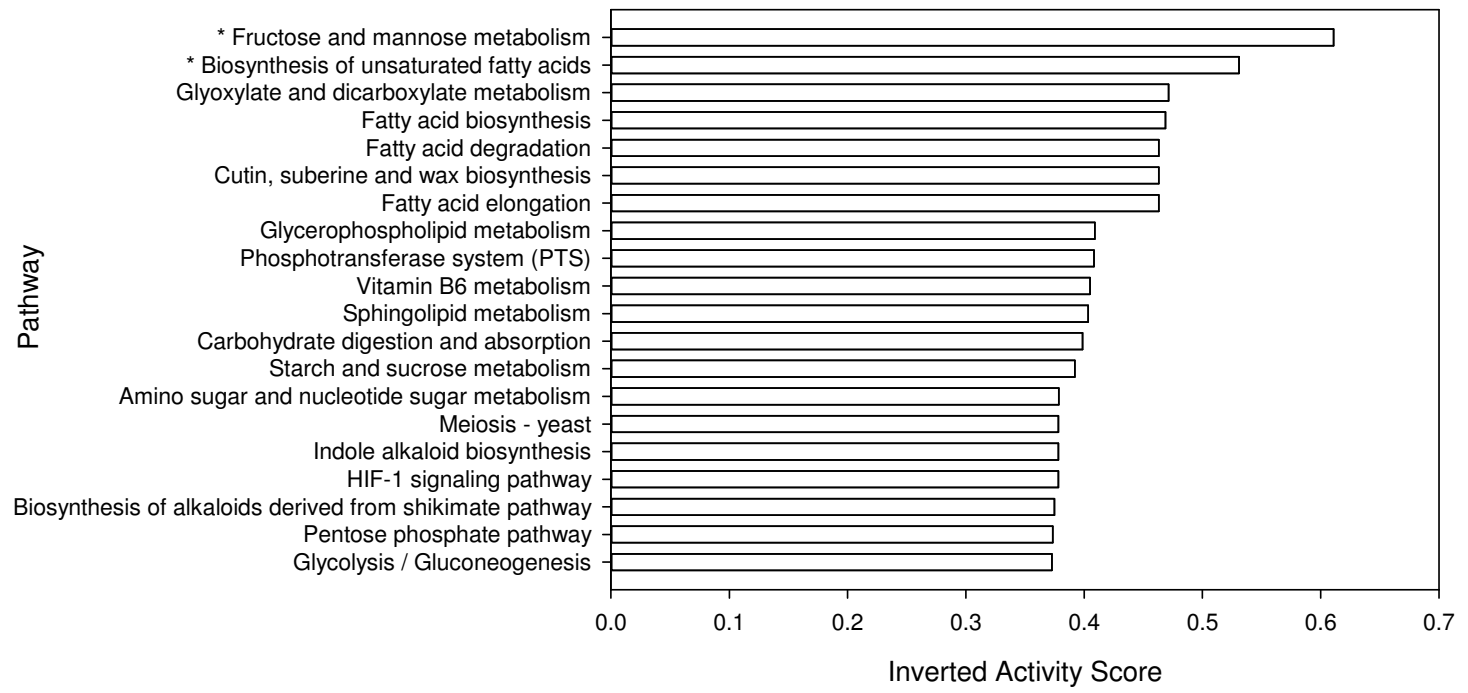

Figure 3.6 Comparison of the activity of key metabolic pathways between seasons in symbionts isolated from A. aureorediata sampled in situ. Values are means of inverted and $\log _{2}$ transformed pathway activity scores of summer relative to winter samples (PAPi activity analysis summer $\mathrm{v}$ winter, t-test, $* \mathrm{p}<0.05)$, highlighting increased activity of numerous pathways during summer.

\subsubsection{Host}

\subsection{Comparison of individual metabolites}

Figure 3.7 shows the top 25 metabolites based on $p$-values (t-test, $n=5$ independent samples) when comparing the metabolite profile of anemones sampled in summer versus those sampled in winter. Of these 25 metabolites, two showed a significant increase in their concentration in summer relative to winter samples when normalised to host 
biomass. These were the amino acids: cis-4-hydroxy-D-proline (FC, 2.0262) and tryptamine (FC, 4.544).

Although not significant, fold change analysis detected large increases in the abundance of the following metabolites in summer relative to winter: (2S,5S)-5-hydroxypiperidine2-carboxylic acid (FC, 3.4844); a compound tentatively identified as N,Ndimethylglycine (FC, 2.9686); 5-androsten-3-beta-ol (FC, 2.9539); methylamine (FC, 2.8877); oleamide (FC, 2.7508); betazole (FC, 2.4564); octadecanamide (FC, 2.414); 4hydroxypyridine (FC, 2.3862); 2-ethylquinoline (FC, 2.3499); erucamide (FC, 2.3371); butyl 2-(ethoxycarbonylamino)-4-methylpentanoate (FC, 2.2963); a compound tentatively identified as L-norleucine, N-propoxycarbonyl-, nonyl ester (FC, 2.2817); a fatty amide tentatively identified as palmitic amide (FC, 2.2631); arachidonic acid (FC, 2.2324); pyridine (FC, 2.0889); N-methylbutan-1-amine (FC, 2.0168); conhydrine (FC, 2.0159) and L-alanine, N-ethoxycarbonyl-, decyl ester (FC, 2.0061). 


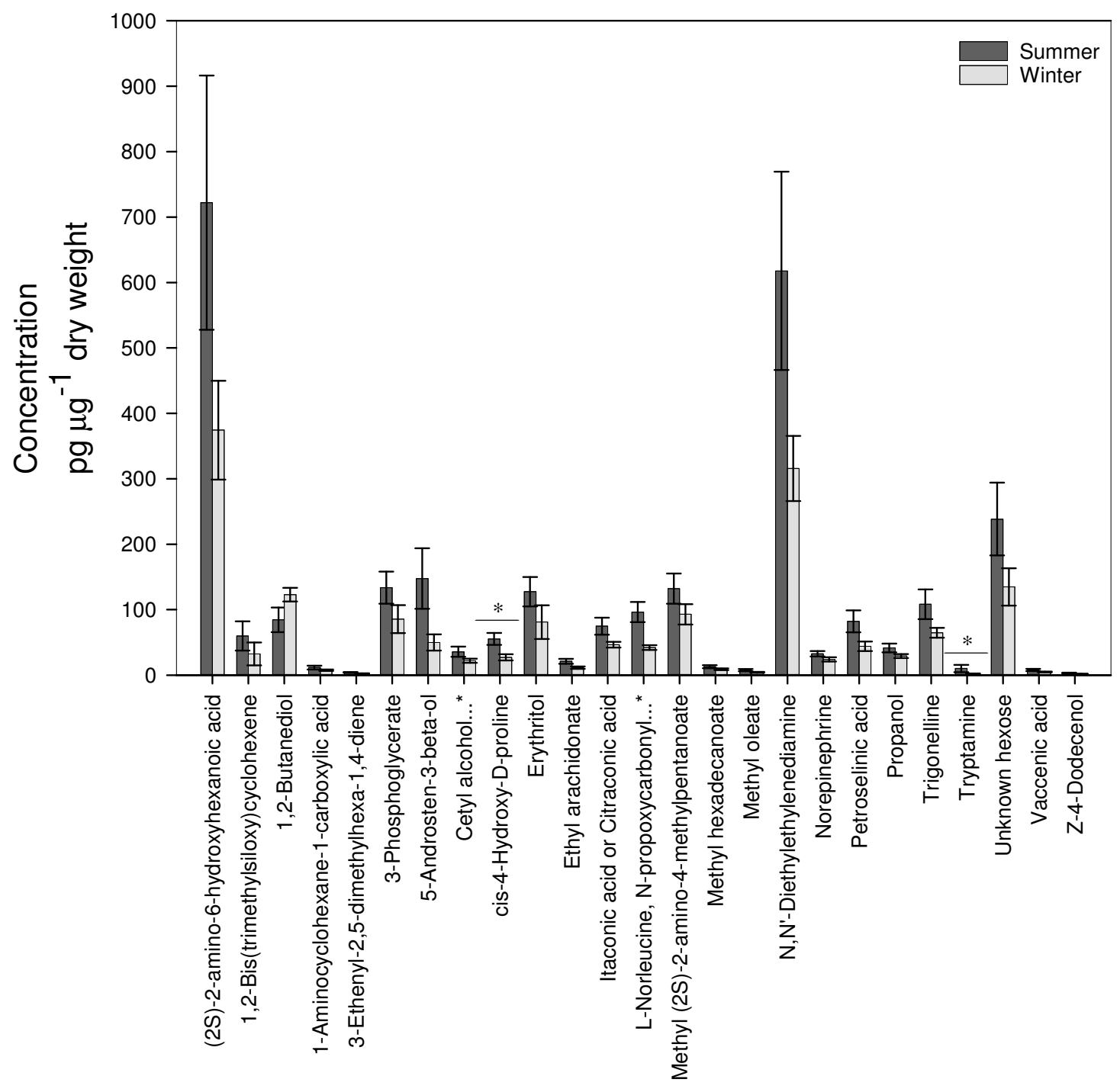

Figure 3.7 Concentration of individual metabolites per $\mu \mathrm{g}$ dry weight in A. aureoradiata sampled in either summer or winter. Values are means $( \pm$ SE) and asterisks indicate significant differences between summer and winter (t-test, $\mathrm{n}=5$, *p $<0.05$ ). (Concatenated names marked (..*), see appendix for full details).

\subsection{Multivariate analysis (PCA)}

From a multivariate perspective, there was no clear difference in the metabolite profile of anemones sampled in summer versus winter (Figure 3.8). Both the summer and winter groups overlap to a large degree on the 2D PCA scores plot, with PC1 explaining $68.3 \%$ 
of the observed variance and PC2 13.1\%. The corresponding loadings plot also shows most of the metabolites tightly grouped with low values on both loadings, indicating that these metabolites are highly correlated between samples, and hence play little role in driving the observed variance. The metabolites with the highest absolute loadings scores are the most influential in driving the observed variance. On the loadings 1 axis these metabolites included the amino acid: L-ornithine; the glycoside: glyceryl monostearate and the unclassified compound: 2-ethylquinoline. On the loadings 2 axis it included the amino acid derivative: alanine, N-methyl-N-butoxycarbonyl-, isohexyl ester; the isocyanate: 2-isocyanato-2-methylpropane and the glycoside: glyceryl monostearate.
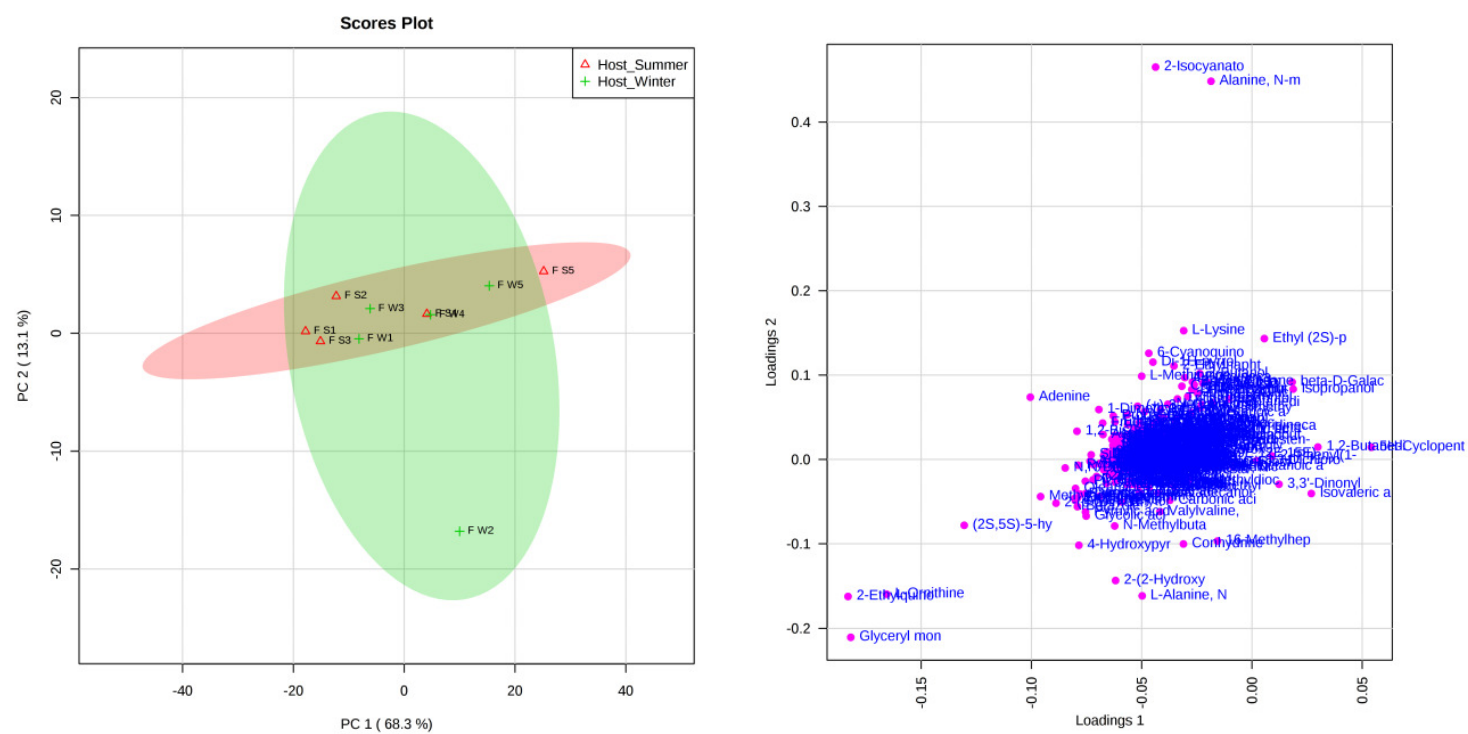

Figure 3.8. PCA scores plot with shaded $95 \%$ confidence intervals (left) and corresponding loadings plot (right) of host anemones collected in summer compared to those collected in winter, providing a multivariate perspective on their metabolite profiles

\subsection{Metabolic pathway response}

Every detected metabolic pathway showed a trend towards decreased activity in anemones sampled in summer relative to anemones sampled in winter. The 20 metabolic pathways expressing the largest absolute change in summer versus winter samples (Figure 3.9) consisted of eight pathways involved in lipid metabolism, three in amino acid metabolism, two in carbohydrate metabolism, two in cell growth and death, one in 
the circulatory system, one in the excretory system, one in glycan biosynthesis, and one in nucleotide metabolism.

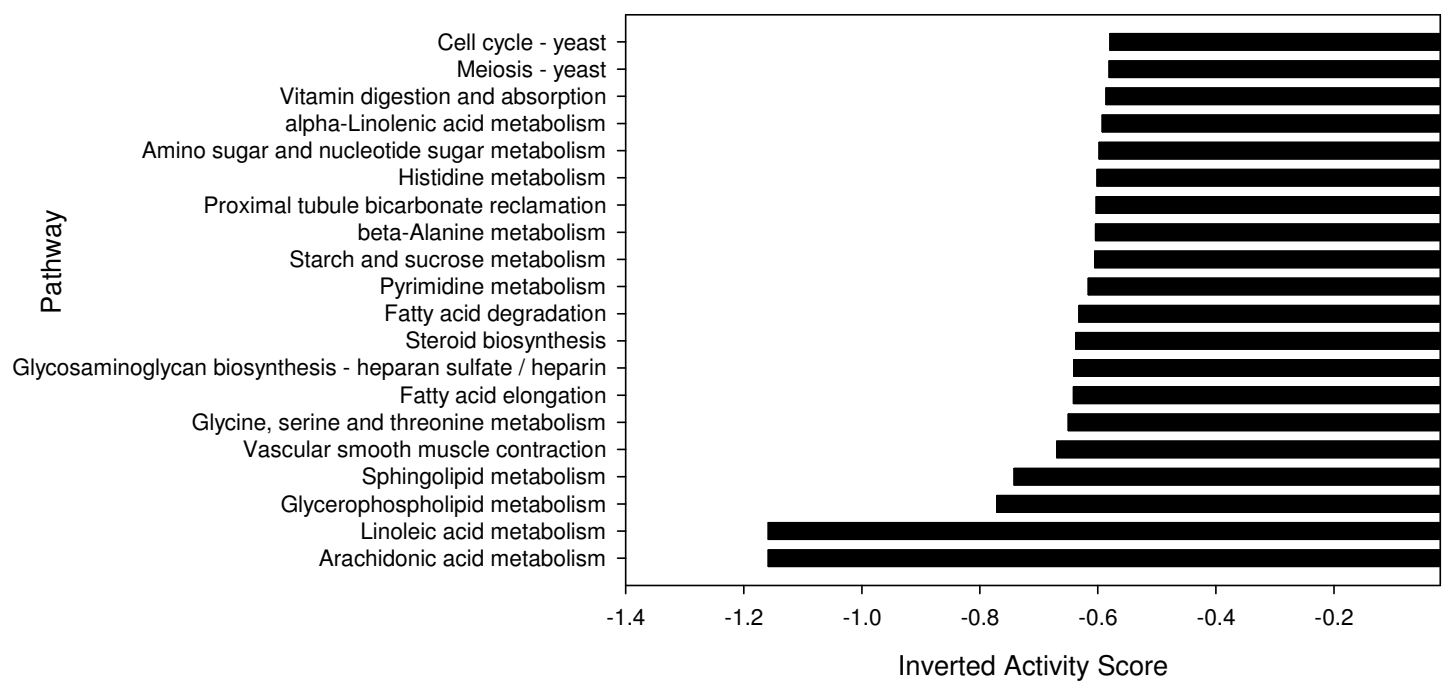

Figure 3.9. Comparison of the activity of key metabolic pathways between seasons in Symbiodinium sp. isolated from A. aureoradiata that were sampled in situ. Values are means of inverted and $\log _{2}$ transformed pathway activity scores in summer relative to winter samples (PAPi activity analysis summer $v s$. winter, t-test, ${ }^{*} \mathrm{p}<0.05$ ), and show a decline in the activities of all pathways in summer relative to winter.

\subsection{The impact of thermal change on the metabolic functioning of A. aureoradiata and their symbionts: an experimental approach}

\subsubsection{State of the symbiosis}

There was no significant change in Symbiodinium cell density, chlorophyll $a$ concentration or chlorophyll $c_{2}$ concentration in the control group over the course of the experiment (Figure 3.10). In the cold treatment, Symbiodinium cell density declined by $640,170.1$ cells $\mathrm{mg}^{-1}$ protein over the course of the six week experiment $\left(\mathrm{t}_{8}=2.863, \mathrm{p}<\right.$ 0.05), while in the hot treatment Symbiodinium cell density declined by $1,146,151.3$ cells 
$\mathrm{mg}^{-1}$ protein over the course of the experiment $\left(\mathrm{t}_{8}=2.770, \mathrm{p}<0.05\right)$. In contrast, chlorophyll $a$ concentration per symbiont cell increased by $0.347 \mathrm{pg} \mathrm{cell}^{-1}$ by the end of the experiment in the hot treatment $\left(\mathrm{t}_{7}=-4.506, \mathrm{p}<0.005\right)$. The chlorophyll $a$ concentration was also $0.319 \mathrm{pg} \mathrm{cell}^{-1}$ greater in the hot treatment compared to the control at the end of the six-week experiment $\left(\mathrm{t}_{7}=4.584, \mathrm{p}<0.005\right)$. Chlorophyll $c_{2}$ concentration increased by $0.096 \mathrm{pg}$ cell $^{-1}$ over the course of the experiment in the hot treatment. $\left(\mathrm{t}_{7}=-4.113, \mathrm{p}<0.01\right)$. The chlorophyll $c_{2}$ concentration was also $0.079 \mathrm{pg}$ cell $^{-}$ 1 greater in the hot treatment compared to the control at the end of the six-week experiment $\left(\mathrm{t}_{7}=3.540, \mathrm{p}<0.01\right)$. 


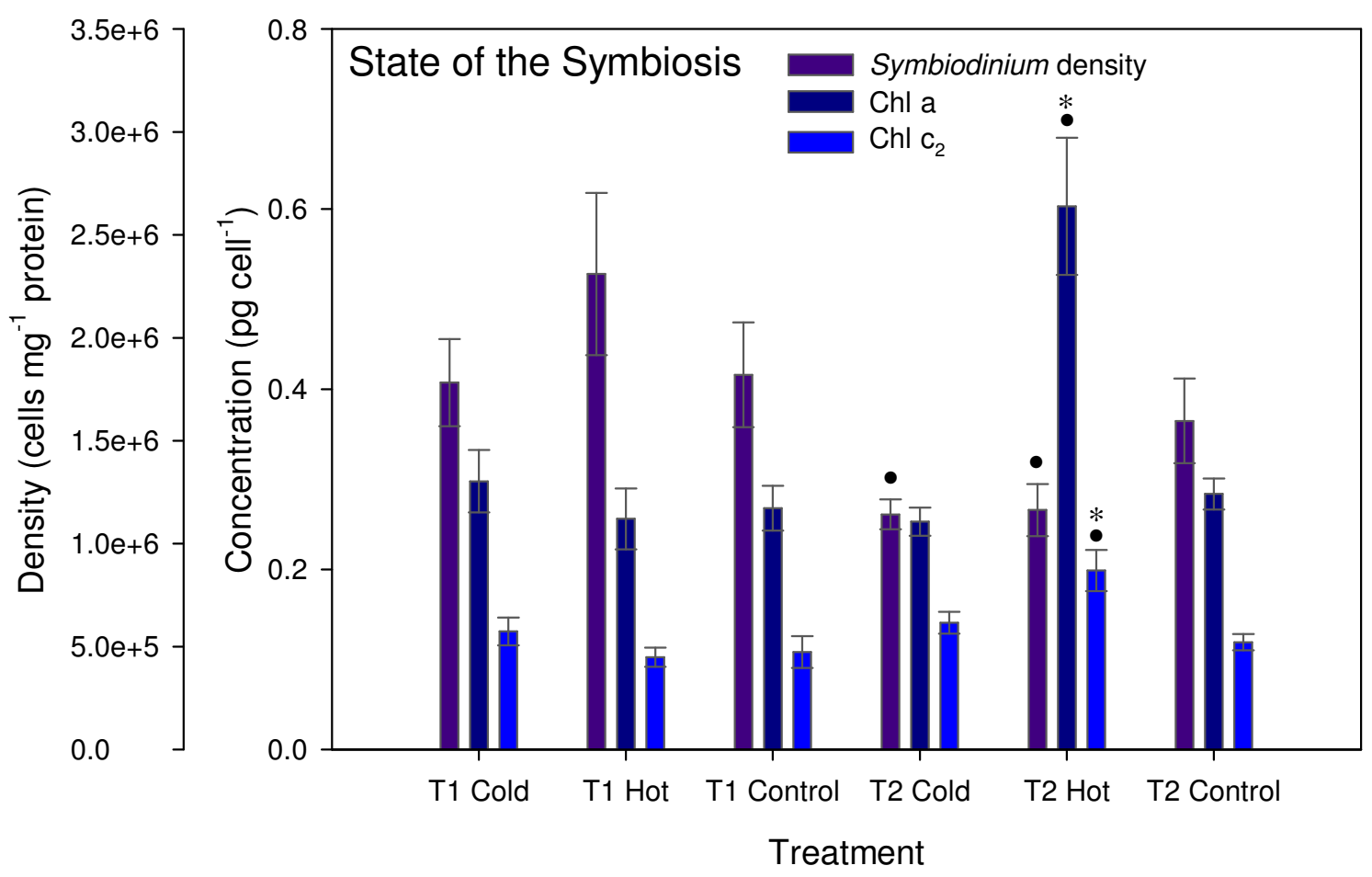

Figure 3.10: Symbiodinium cell density and chlorophyll $a$ and $c_{2}$ concentrations per symbiont cell in A. aureoradiata under in response to experimental conditions. Prior to initiation of the experiment all tanks were kept at $15^{\circ} \mathrm{C}$ and are labelled as $\mathrm{T} 1 \mathrm{cold}, \mathrm{T} 1$ hot and T1 control, respectively. Following six weeks at treatment temperatures tanks are labelled $\mathrm{T} 2$; cold $\left(8^{\circ} \mathrm{C}\right)$, hot $\left(28^{\circ} \mathrm{C}\right)$ and control $\left(15^{\circ} \mathrm{C}\right)$. Values are means $( \pm \mathrm{SE})$ and asterisks denote significant differences between groups compared using a t-test, (T1 cold vs. T2 cold, T1 hot vs. T2 hot and T1 control vs. T2 control, t-test, $\bullet \mathrm{P}<0.05)$, (T2 cold and $\mathrm{T} 2$ hot $v s$. $\mathrm{T} 2$ control, t-test, $* \mathrm{P}<0.05)$.

\subsubsection{Changes in the metabolite profile}

\subsubsection{Impact of thermal change on the symbiont metabolite profile}

\subsection{Comparison of individual metabolites}

Figure 3.11 shows the top 25 metabolites based on p-values (t-test, $n=5$ independent samples) when comparing cold $\left(8^{\circ} \mathrm{C}\right)$ and hot $\left(28^{\circ} \mathrm{C}\right)$ to control $\left(15^{\circ} \mathrm{C}\right)$ symbionts following a six-week treatment period. In the cold treated group (Figure 3.11 A) three of 
these metabolites showed a significant difference. These compounds included the parent compound of the amphetamine group: amphetamine, the amino acid: beta-alanine and the cyclic alcohol: cyclopentanol.

Although not significant, in the cold treated group large increases were detected via fold change analysis in the abundance of beta-D-glucose (FC, 3.2985) and pentyl 2-(2methoxyethoxycarbonylamino)pentanoate (FC, 2.5968). Large reductions in the abundance of 1,4-butanediamine (FC, 0.26777) and sucrose (FC, 0.48508) were also observed.

In the heat treated group (Figure 3.11 B), two metabolites showed a significant difference. As in the cold treated group, the amino acid beta-alanine was one of these metabolites. However, in contrast to the cold treated group, in the heat treated group the concentration increased. The other metabolite was the sugar derivative beta-1galactopyranoside, methyl 6-deoxy-2,3,4-tris-O-(trimethylsilyl) (FC, 0.43514). Interestingly, this metabolite showed a reduction in both the cold and heat treated groups.

Although not significant, in the heat treated group, fold change analysis detected large reductions in the abundance of alanine, N-methyl-n-butoxycarbonyl-, isohexyl ester (FC, 0.34704); 1,4-butanediamine (FC, 0.35161) and a sugar tentatively identified as alpha-Dmannopyranose (FC, 0.49917). 


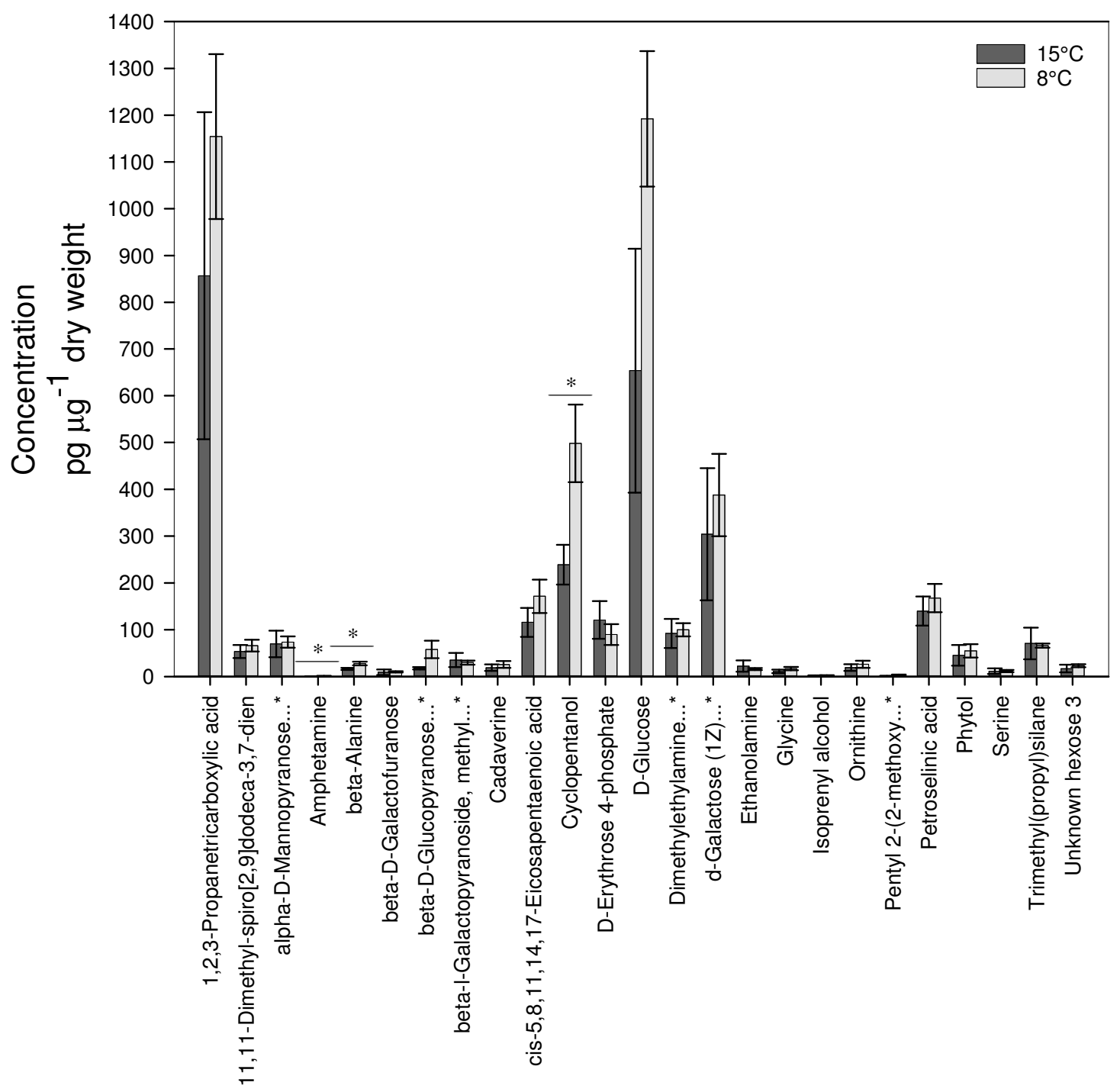




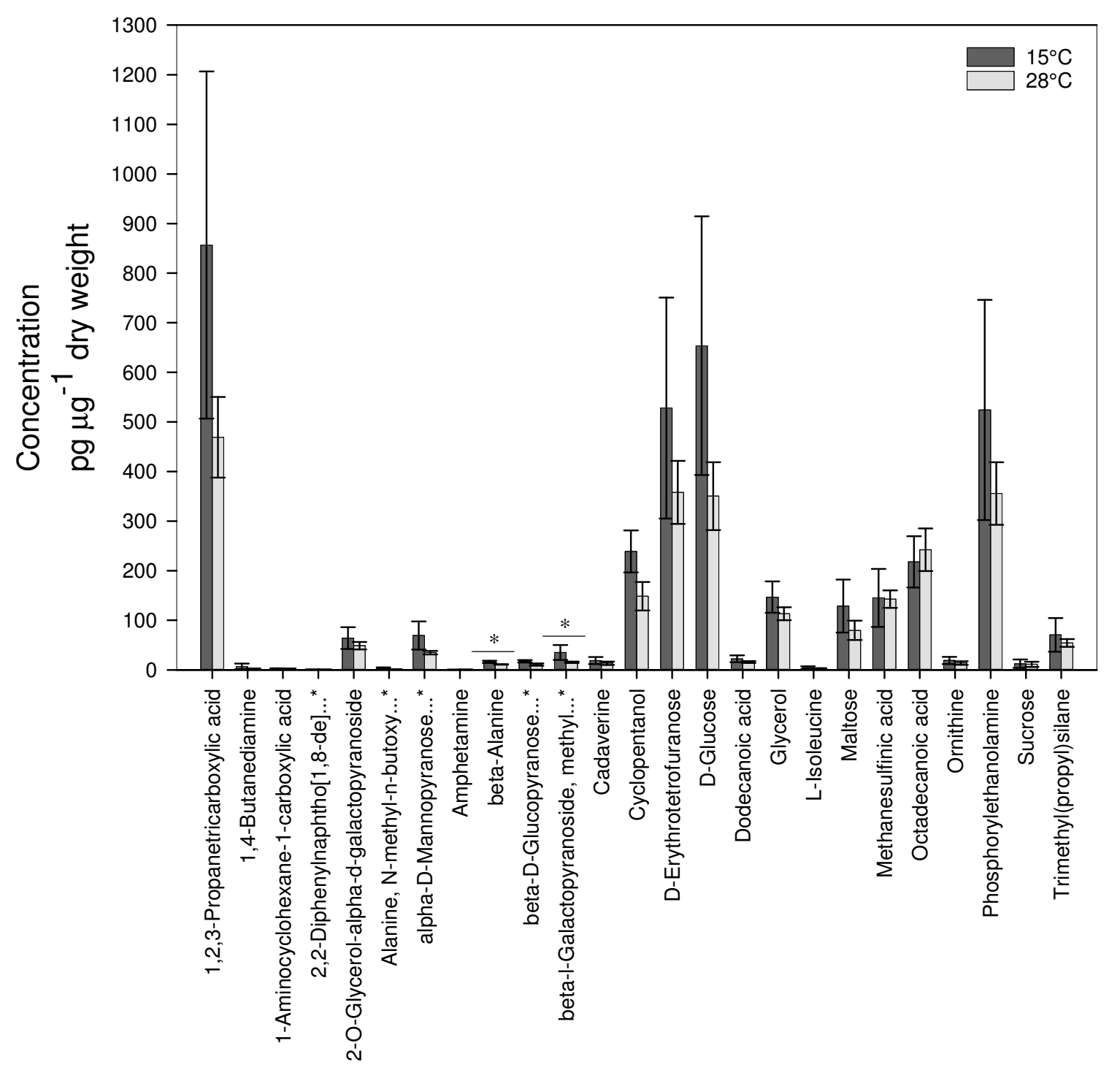

Figure 3.11. Concentration of individual metabolites per $\mu \mathrm{g}$ dry weight in cold (A) and heat treated (B) symbionts, Symbiodinium sp. isolated from A. aureoradiata, relative to controls $\left(15^{\circ} \mathrm{C}\right)$ over a 6 -week period. Values are means $( \pm \mathrm{SE})$ and asterisks indicate significant differences between treatment and control (t-test, $\mathrm{n}=5, * \mathrm{p}<0.05$ ). (Concatenated names marked (...*), see appendix for full details). 


\subsection{Multivariate analysis (PCA)}

From a multivariate perspective, there was no clear effect of cold or heat treatment on the symbiont metabolite profile (Figure 3.12). The cold treated group overlaps with the control group to a large degree on the 2D PCA scores plot, with PC1 explaining $71.7 \%$ of the observed variance and PC2 $10.4 \%$ (Figure 3.12A). However, both the cold and control groups display a high level of within group variability, mostly along the PC1 axis. The corresponding loadings plot also shows a significant spread in the metabolites, with many metabolites showing large absolute values on the loadings 1 axis, indicative of the high degree of variability explained by that axis. The metabolites with the highest absolute loadings scores are the most influential in driving the variance observed in the scores plot. On the loadings 1 axis these metabolites included the sugars: sucrose, beta-Dgalactofuranose and ribose; the secondary amine: diethylamine; and the amino acids: phenylalanine; L-isoleucine and serine. On the loadings 2 axis it includes the parent compound of the amphetamine group: amphetamine; the sugar: beta-D-glucose and the unclassified compounds: pentyl 2-(2-methoxyethoxycarbonylamino)pentanoate; silane, dimethyl(4-phenylphenoxy)undecyloxy-.

The heat treated group also overlaps the control group to a high degree on the 2D PCA scores plot (Figure 3.12 B), with PC1 explaining $72.5 \%$ of the observed variance and PC2 $11 \%$. However, both the heat treated and control groups display a high degree of within group variability, mostly along the PC1 axis. The corresponding loadings plot also shows a significant spread in the metabolites, with many metabolites showing large absolute values on the loadings 1 axis, indicative of the high degree of variability explained by that axis. The metabolites with the highest absolute loadings scores are the most influential in driving the variance observed in the scores plot. On the loadings 1 axis these metabolites included the sugars: sucrose; beta-D-galactofuranose and ribose; the amino acid: L-valine and the amine: 1,4-butanediamine. On the Loadings 2 axis it included the sugar: beta- D-glucose and the unclassified compounds: pentyl 2-(2methoxyethoxycarbonylamino)pentanoate and alanine, N-methyl-n-butoxycarbonyl-, isohexyl ester. 
A
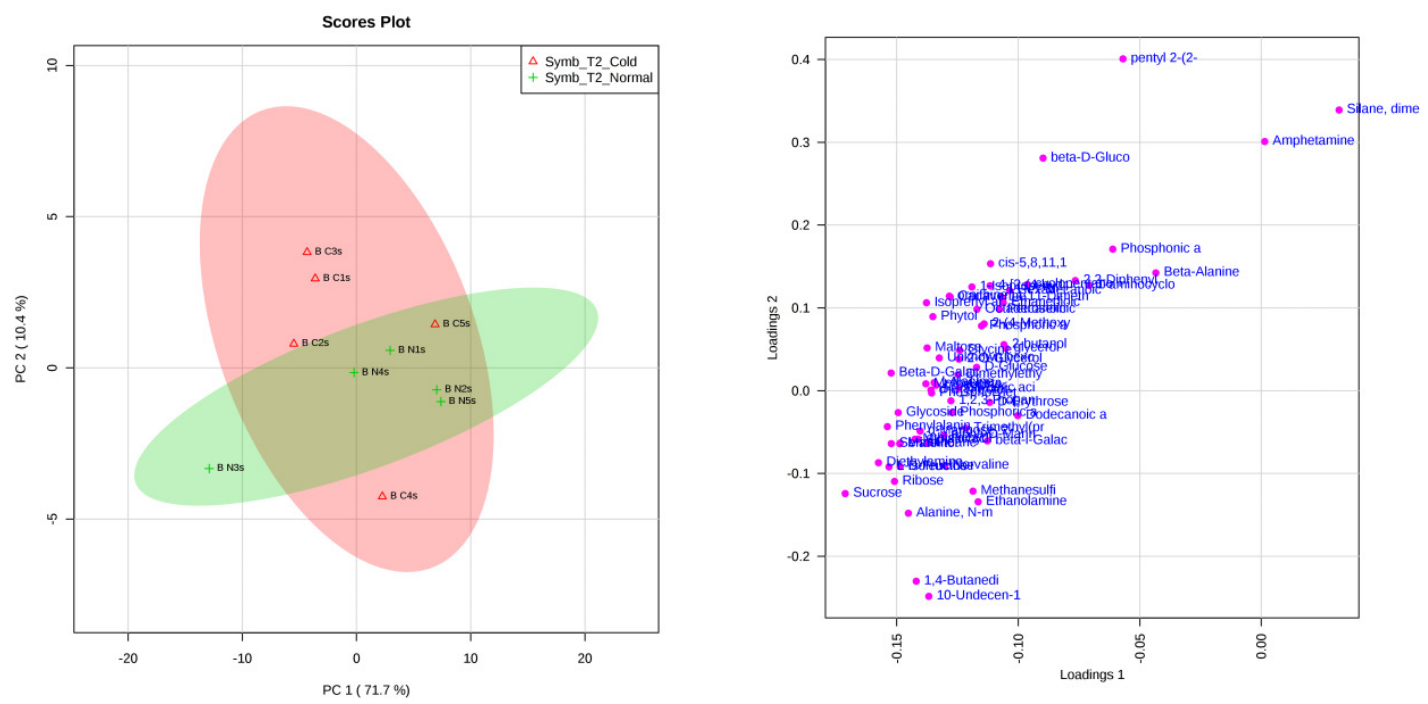

B
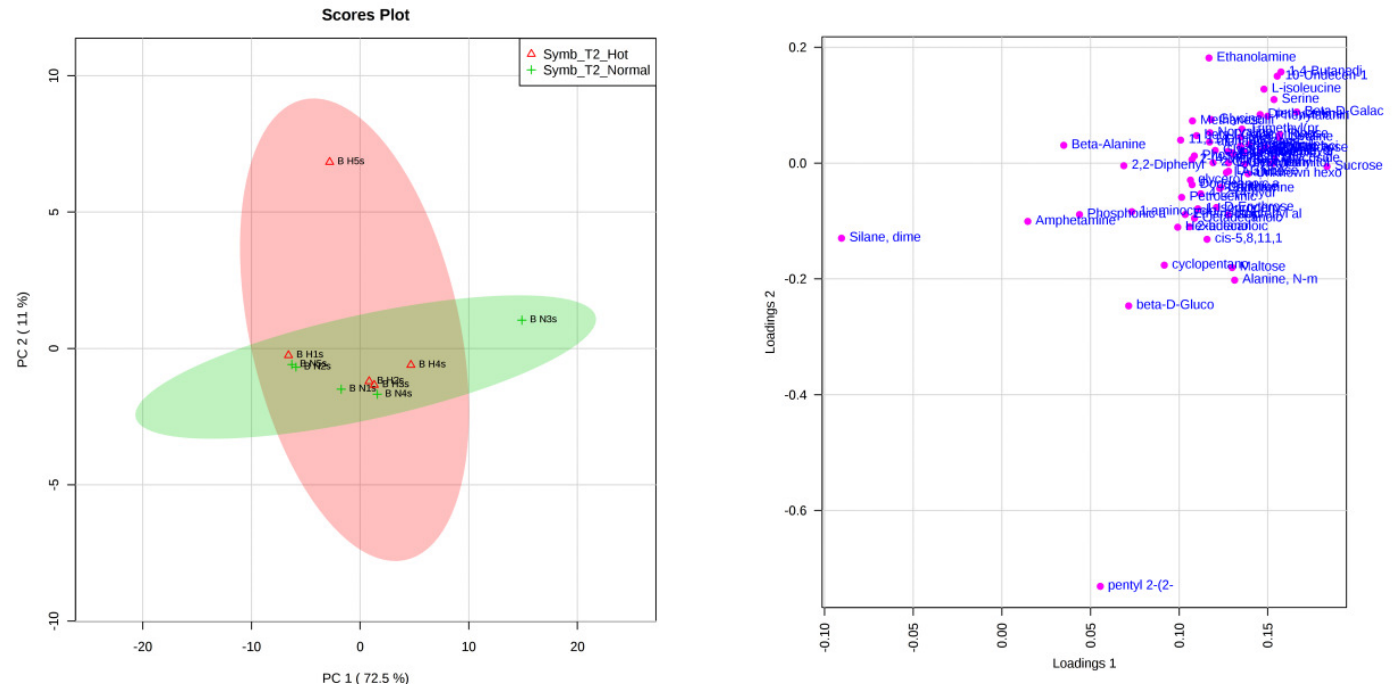

Figure 3.12 PCA scores and corresponding loadings plot for symbionts isolated from $A$. aureoradiata exposed to cold treatment (A) and heat treatment (B) over a 6-week period, relative to controls $\left(15^{\circ} \mathrm{C}\right)$. Symb_T2_Cold, $=$ Week 6 cold; Symb_T2_Hot $=$ Week 6 hot; Symb_T2_Normal $=$ Week 6 control. Shaded regions represent 95\% confidence intervals 


\subsection{Metabolic pathway response}

Most of the detected metabolic pathways showed a trend towards decreased activity in symbionts exposed to reduced temperature $\left(8^{\circ} \mathrm{C}\right)$ over a 6-week period (Figure 3.13 A). Pathway activity decreased in all but one of the 20 metabolic pathways expressing the largest absolute change relative to controls. This included five pathways involved in carbohydrate metabolism, four in amino acid metabolism, two in the metabolism of cofactors and vitamins, two in the digestive system, two in nucleotide metabolism, two in the biosynthesis of secondary metabolites, one in cell growth and death, one in environmental information processing, and one in membrane transport. The only pathway showing an increase in activity was the phosphonate and phosphinate metabolism pathway, which is an amino acid metabolism pathway.

In the heat treated $\left(28^{\circ} \mathrm{C}\right)$ group, most of the detected metabolic pathways showed a trend towards increased activity (Figure 3.13 B). Pathway activity increased in all of the 20 metabolic pathways expressing the largest absolute change relative to controls. This included six pathways involved in carbohydrate metabolism, four in amino acid metabolism, four in the biosynthesis of secondary metabolites, two in membrane transport, one in cell growth and death, one in the digestive system, one in nucleotide metabolism and one in environmental information processing. 


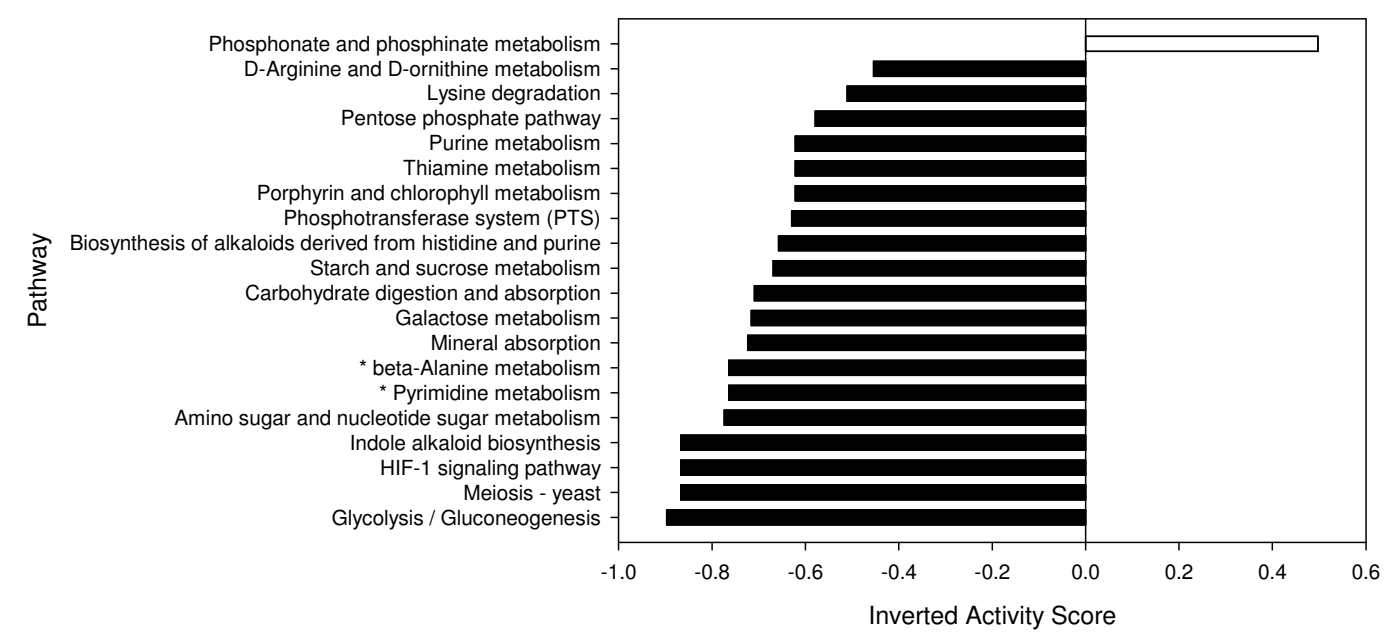

B

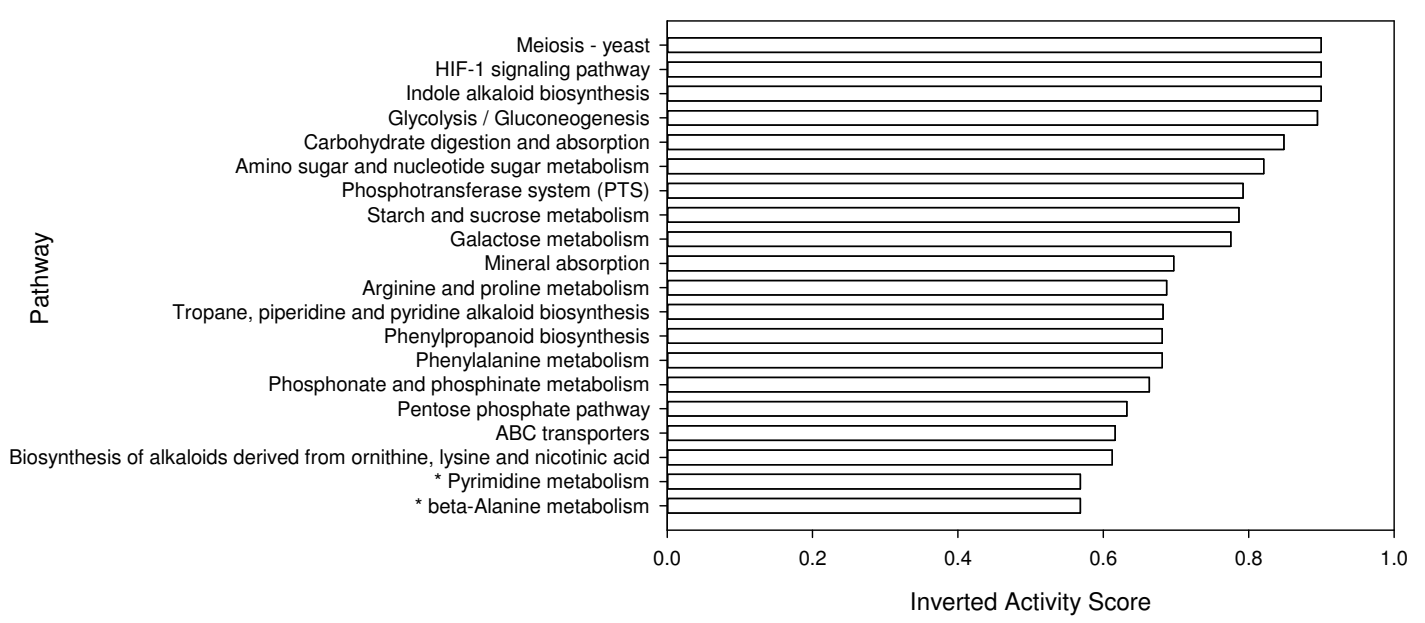

Figure 3.13 Effect of six weeks of $(\mathrm{A})$ cold $\left(8^{\circ} \mathrm{C}\right)$ and $(\mathrm{B})$ heat $\left(28^{\circ} \mathrm{C}\right)$ treatment on the activity of key symbiont metabolic pathways. Values are means of inverted and $\log _{2}$ transformed pathway activity scores in cold and hot treated groups relative to controls (PAPi activity analysis T6 cold and T6 hot vs. T6.control, t-test, ${ }^{*} \mathrm{p}<0.05$ ) 


\subsubsection{Impact of thermal change on the host metabolite profile}

\subsection{Comparison of individual metabolites}

Figure 3.14 shows the top 25 metabolites based on p-values (t-test, $n=5$ independent samples) when comparing cold treated $\left(8^{\circ} \mathrm{C}\right)$ and heat treated $\left(28^{\circ} \mathrm{C}\right)$ to control $\left(15^{\circ} \mathrm{C}\right)$ anemones following a six-week treatment period. In the cold treated group (Figure 3.14 A), seven metabolites showed a significant difference. These compounds included the organic acid: propanedioic acid; the fatty acid: isovaleric acid; the glycoside: methyl betaD-glucopyranoside (FC, 0.40035); the alcohols: 2-butanol and 3-methoxybutan-2-ol; and the unclassified compounds: 3,3'-dinonyl-2,2'-bithiazolidine (FC,4.261); and N-[(E)-2,2dimethylpropylidene] (trimethyl) silanamine or N-ethylformamide.

Although not significant, in the cold treated group, large reductions in abundance were also detected via fold change analysis for 2-Isocyanato-2-methylpropane (FC, 0.27993); Alanine, N-methyl-N-butoxycarbonyl-, isohexyl ester (FC, 0.28105); L-lysine (FC, 0.41055); $\quad$ ethyl (2S)-pyrrolidine-2-carboxylate $\quad(\mathrm{FC}, \quad 0.44794) ; \quad 1,2-$ bis(trimethylsiloxy)cyclohexene (FC, 0.4607); di-1H-pyrrol-1-yl-isopropenylsilane (FC, 0.46374) and sucrose (FC, 0.47645). Large increases were observed in the abundance of 16-methylheptadecane-1,2-diol (FC, 3.7079); 2-(2-hydroxy-1-methylethyl)-1H-isoindole1,3(2H)-dione (FC, 2.8299); L-alanine, N-ethoxycarbonyl-, decyl ester (FC, 2.732); 4hydroxypyridine (FC, 2.3317) and ethylene glycol (FC, 2.1246).

In the heat treated group (Figure 3.14 B), ten metabolites showed a significant difference, none of which were shared with the metabolites that changed significantly in the cold treated group. These compounds included the amino acid: beta-aminoisobutyric acid; the parent compound of the amphetamine group: amphetamine; the phenyl acetic acid derivative: benzeneacetic acid; the sugar acid: erythronic or threonic acid; the amine: ethylamine; the fatty acid: itaconic or citraconic acid; the glycoside: methyl beta-Dglucopyranoside (FC, 2.0388); the isothiocyanate: methyl isothiocyanate; the fatty acid derivative: an unknown inositol stereoisomer and the unclassified compound: 2ethylnaphtho[1,8-de][1,3,2]dioxaborinine.

Although not significant, in the heat treated group, fold change analysis detected large increases in abundance of L-alanine, N-ethoxycarbonyl-, decyl ester (FC, 3.1251), which 
also increased in the cold treated group; conhydrine (FC, 2.3943); ethylene glycol (FC, 2.0196), which also increased in the cold treated group and sucrose (FC, 2.0114).

A

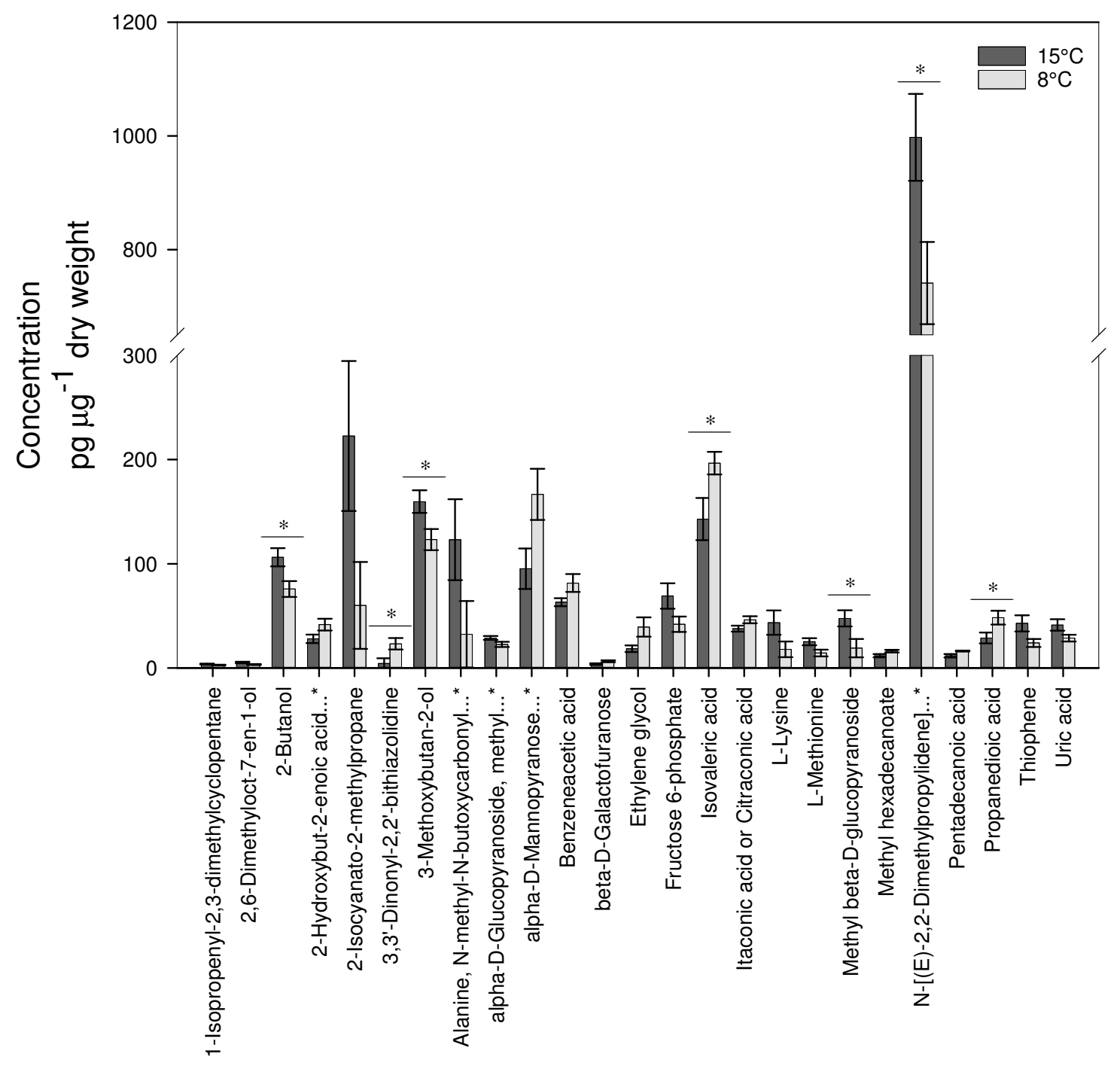




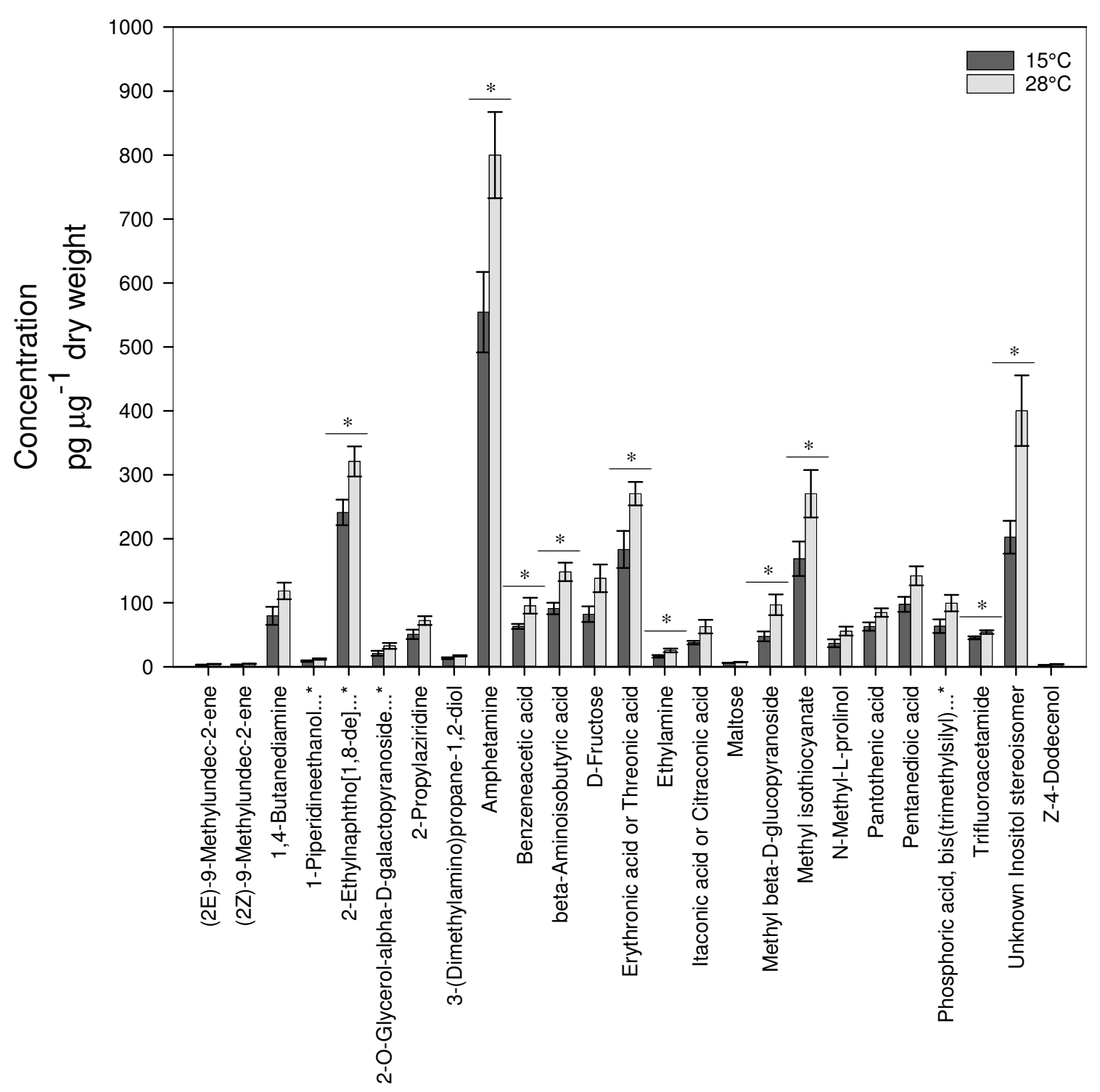

Figure 3.14 Concentration of individual metabolites per $\mu \mathrm{g}$ dry weight in A. aureoradiata exposed to cold treatment (A) and heat treatment (B) over a 6-week period, relative to controls $\left(15^{\circ} \mathrm{C}\right)$. Values are means $( \pm \mathrm{SE})$ and asterisks indicate significant differences between treatment and control ( $\mathrm{t}$-test, $\mathrm{n}=5$, $* \mathrm{p}<0.05$ ). (Concatenated names marked $\left(. .{ }^{*}\right)$, see appendix for full details). 


\subsection{Multivariate analysis (PCA)}

From a multivariate perspective, there was no clear effect of cold or heat treatment on the host metabolite profile (Figure 3.15). Both the cold and control groups overlap to a large degree on the 2D PCA scores plot, with PC1 explaining $43.2 \%$ of the observed variance and PC2 26.4\% (Figure 3.15 A). However, the cold-treated group does display much less within group variability than the control group. The corresponding loadings plot also shows most of the metabolites tightly grouped with low values on both loadings, indicating that these metabolites are highly correlated between samples, and hence play little role in driving the observed variance. The metabolites with the highest absolute loadings scores are the most influential in driving the observed variance. On the loadings 1 axis these metabolites included the amino acid: L-tryptophan; the purine base: adenine; the fatty amides: octadecanamide, oleamide and a compound tentatively identified as palmitic amide (see appendix for details) and the unclassified compound: 2ethylquinoline. On the Loadings 2 axis it included the pyridine derivative: 4hydroxypyridine; the sterol: 5-androsten-3-beta-ol and the unclassified compounds: 3,3'dinonyl-2,2'-bithiazolidine; and 2-(2-hydroxy-1-methylethyl)-1H-isoindole-1,3(2H)dione.

The heat treated group also overlaps with the control group to a large degree on the 2D PCA scores plot, with PC1 explaining $42.4 \%$ of the observed variance and PC2 $31.9 \%$. (Figure 3.15 B). The corresponding loadings plot also shows most of the metabolites tightly grouped, with low values on both loadings, indicating that these metabolites are highly correlated between samples, and hence play little role in driving the observed variance. The metabolites with the highest absolute loadings scores are the most influential in driving the variance observed in the scores plot. All of the metabolites with high loadings values corresponded with metabolites that also had high loadings values in the cold treated group. Metabolites with high values on both the loadings 1 and 2 axes included the pyridine derivative: 4-hydroxypyridine and the unidentified compounds 2(2-hydroxy-1-methylethyl)-1H-isoindole-1,3(2H)-dione and 3,3'-dinonyl-2,2'bithiazolidine. Additionally, with a high value on just the loadings 2 axis was the sterol: 5androsten-3-beta-ol. 
A
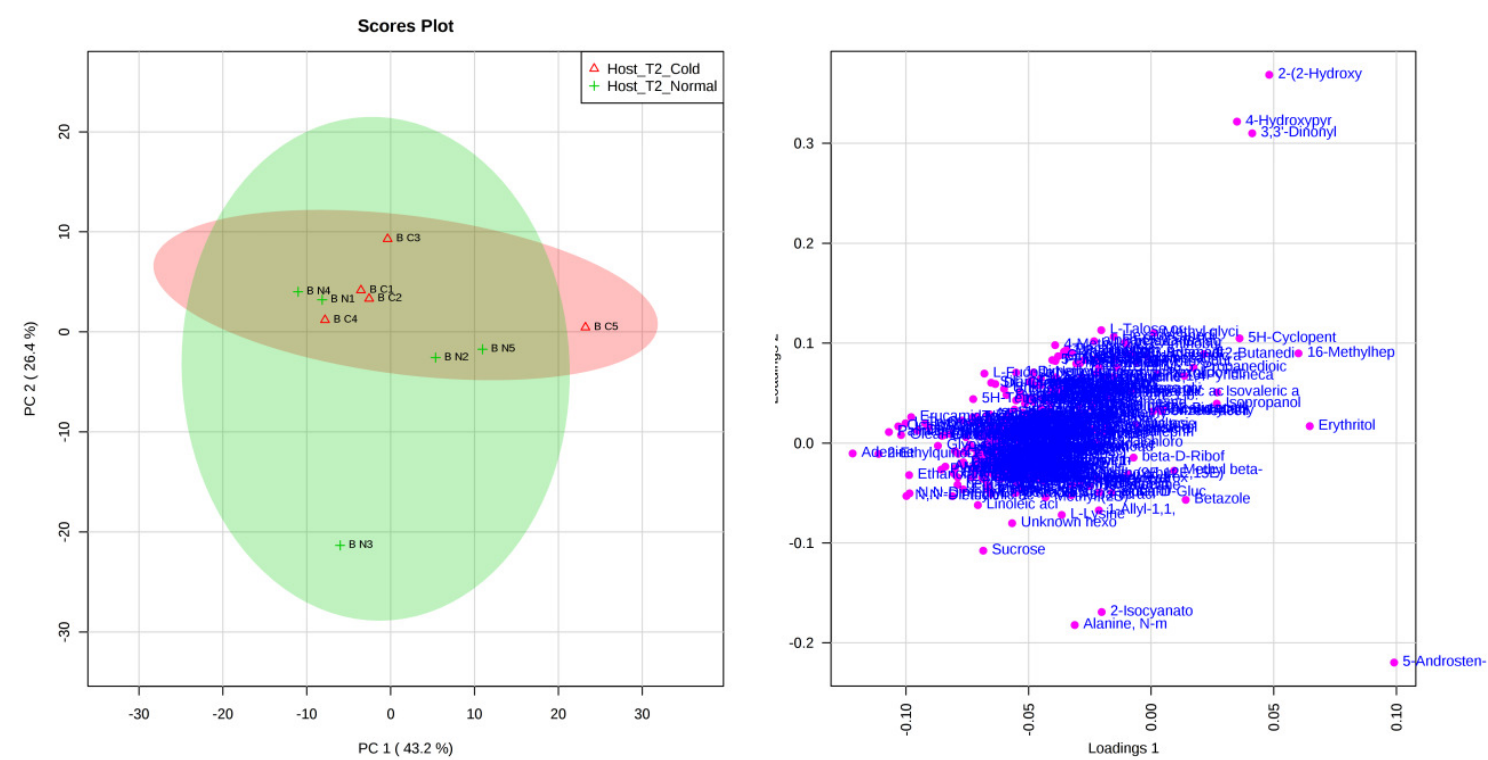

B
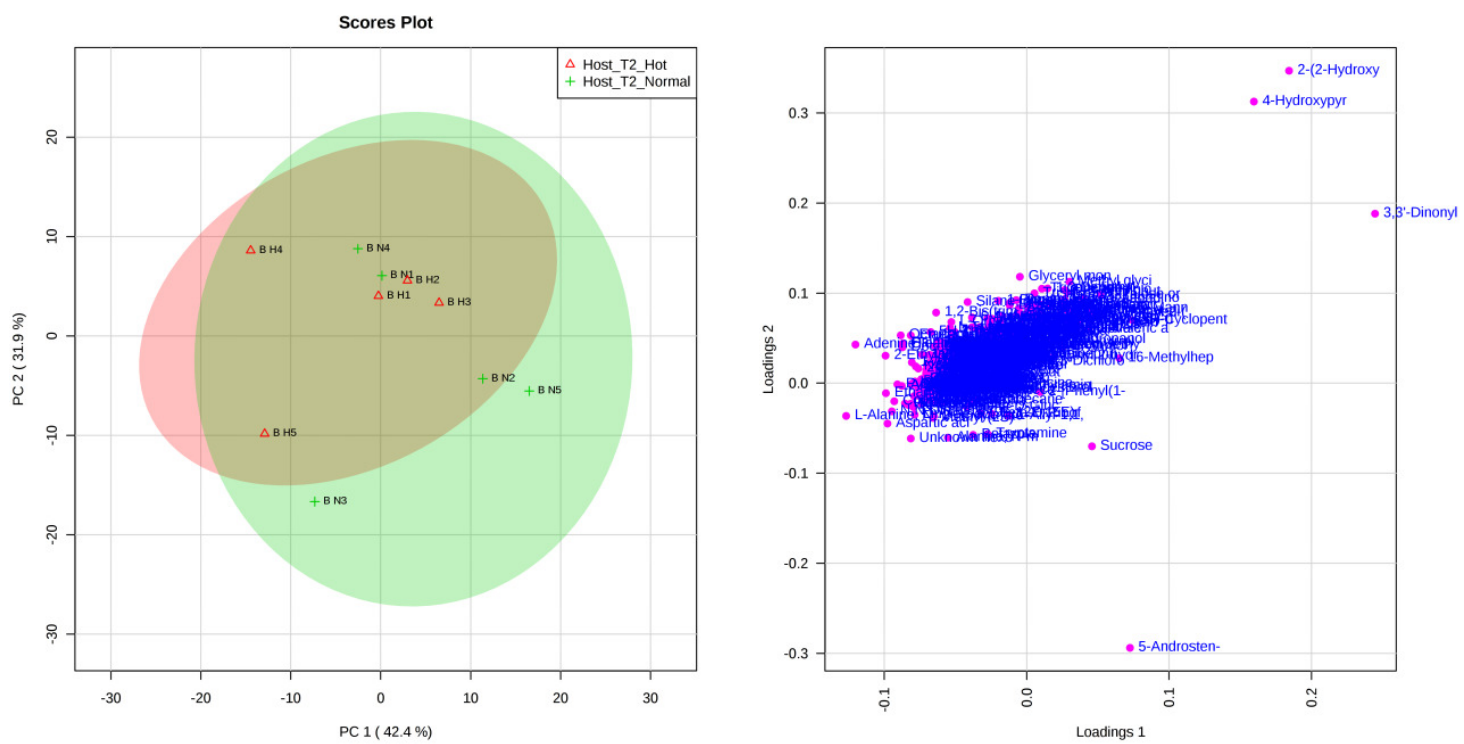

Figure 3.15 PCA scores and corresponding loadings plot for $A$. aureoradiata exposed to cold treatment $(\mathrm{A})$ and heat treatment $(\mathrm{B})$ over a 6-week period, relative to controls $\left(15^{\circ} \mathrm{C}\right)$. Host_T2_Cold, $=$ Week 6 cold; Host_T2_Hot $=$ Week 6 hot; Host_T2_Normal $=$ Week 6 control. Shaded regions represent $95 \%$ confidence intervals. 


\subsection{Metabolic pathway response}

Most of the detected metabolic pathways showed a trend towards increased activity in anemones exposed to reduced temperatures $\left(8^{\circ} \mathrm{C}\right)$ over a 6-week period (Figure 3.16 A). Pathway activity increased in all but three of the 20 metabolic pathways that expressed the largest absolute change relative to controls. This included six pathways involved in lipid metabolism, three in amino acid metabolism, two in nucleotide metabolism, two in the metabolism of co-factors and vitamins, one in glycan biosynthesis, one in carbohydrate metabolism, one in energy metabolism, and one in cell growth and death. Of the three pathways in which decreased activity was detected, one was involved in lipid metabolism, one in carbohydrate metabolism and one in glycan biosynthesis.

In anemones exposed to elevated temperature $\left(28^{\circ} \mathrm{C}\right)$ over a six-week period most of the detected metabolic pathways showed a trend towards decreased activity (Figure 3.16 B). Pathway activity declined in all of the 20 metabolic pathways expressing the largest absolute change relative to controls. This included four pathways involved in amino acid metabolism, three in carbohydrate metabolism, two in lipid metabolism, two in energy metabolism, two in the metabolism of co-factors and vitamins, two in glycan biosynthesis, one in the digestive system, one in the excretory system, one in nucleotide metabolism and one in cell growth and death. 


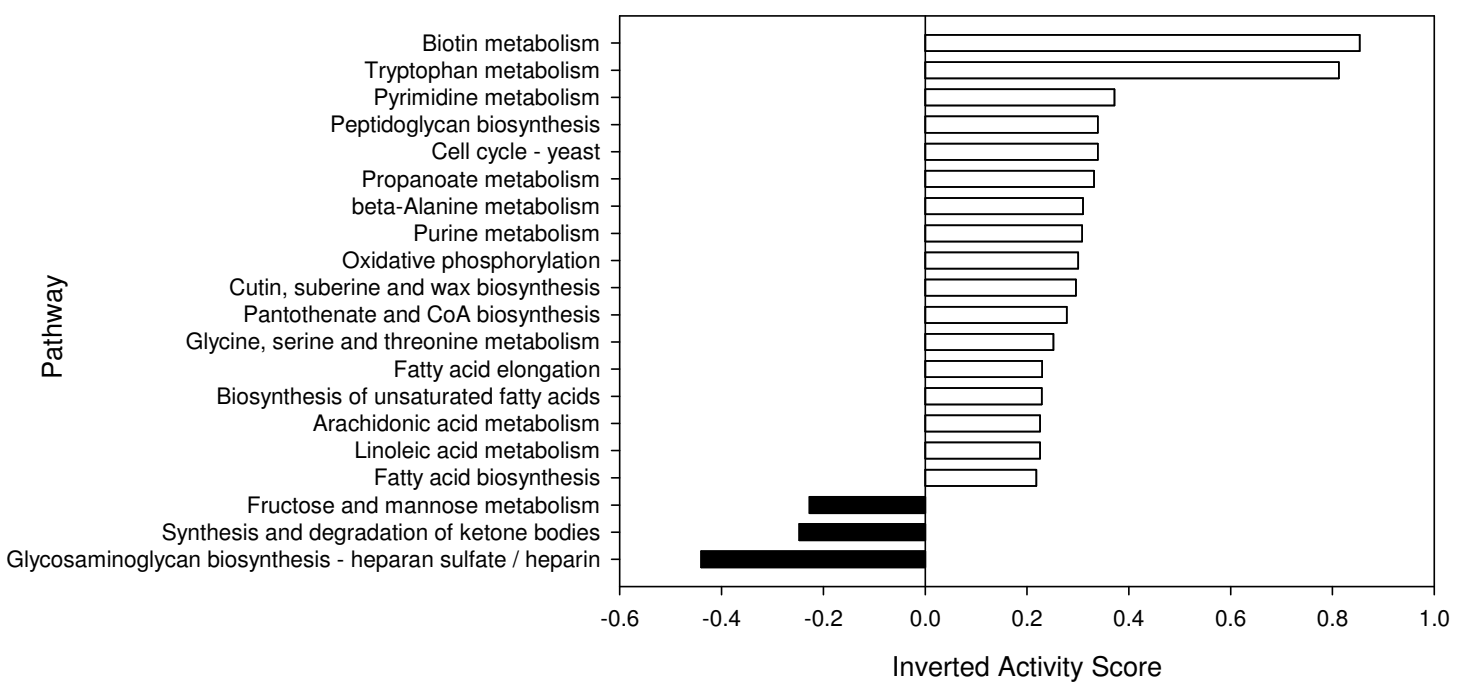

B

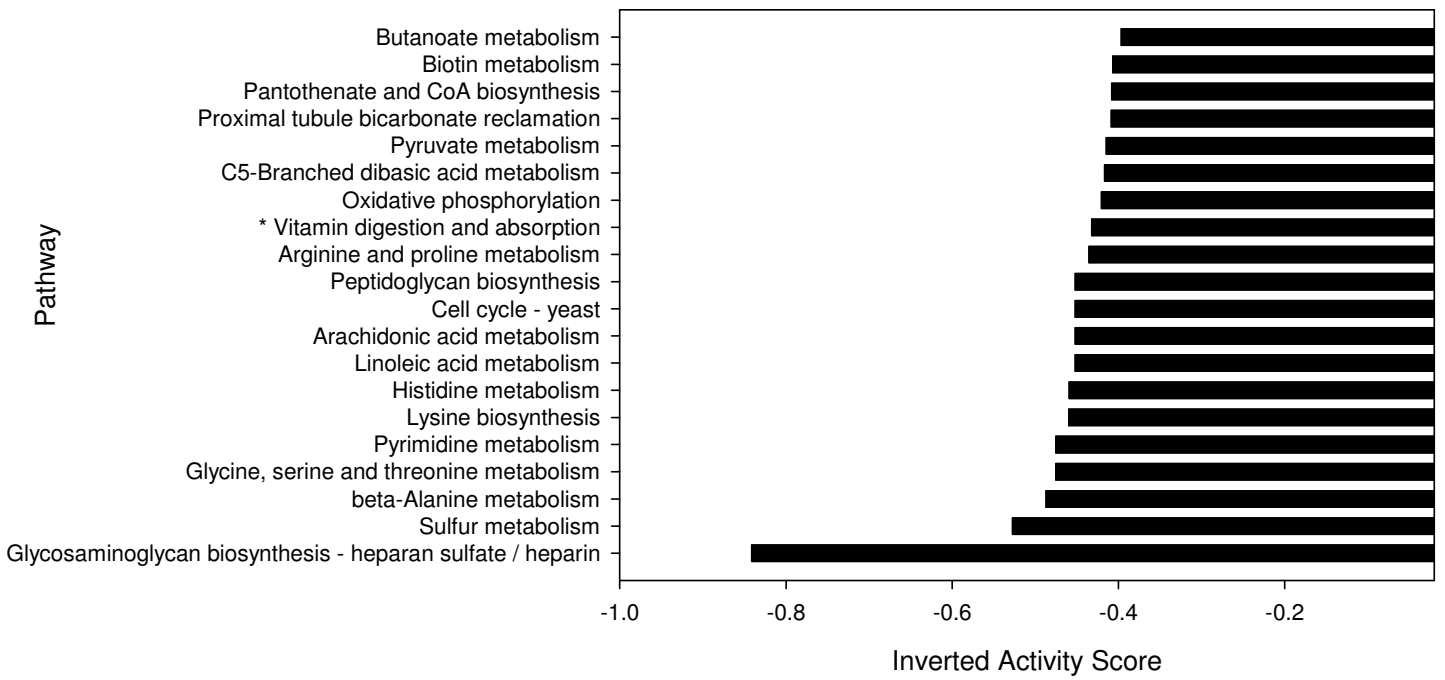

Figure 3.16 Effect of six weeks of $(\mathrm{A})$ cold $\left(8^{\circ} \mathrm{C}\right)$ and $(\mathrm{B})$ heat $\left(28^{\circ} \mathrm{C}\right)$ treatment on the activity of key host metabolic pathways. Values are means of inverted and $\log _{2}$ transformed pathway activity scores in cold and heat treated groups relative to controls (PAPi activity analysis T6 cold and T6 hot $v s$. T6. control, t-test, * $\mathrm{p}<0.05$ ). 


\section{Chapter 4: Discussion}

This work provides new insight into the metabolic functioning of the temperate sea anemone Anthopleura aureoradiata and its dinoflagellate symbionts. Alterations to metabolic functioning were observed both in the anemone's natural habitat in response to season, and in the laboratory in response to thermal change. Response patterns were similar in response to winter and cold, and summer and heat, though the responsive metabolites were not necessarily the same between field and lab situations. Responsive metabolites could be grouped as either organic acids, fatty acids, amino acids or sugars. Additionally, large fold-changes were detected in the activity levels of a range of metabolic pathways, especially those involved in central carbohydrate metabolism, energy metabolism, amino acid metabolism and lipid metabolism. In the host, most pathways were down-regulated in summer/warm conditions but up-regulated in winter/cold conditions, while pathways in the symbionts did the opposite. The potential roles of these various metabolites and pathways in the maintenance of cellular homeostasis and responses to thermal extremes will be discussed further below.

\subsection{The impact of environmental variability on symbiotic state}

Interestingly, no significant changes were observed in a set of key indicators of the symbiotic state between seasons, including Symbiodinium cell density and the concentration of chlorophyll $a$ and $c_{2}$ per symbiont cell. This is likely largely due to the known capacity of temperate cnidarian-dinoflagellate symbioses to maintain stable symbiotic relationships in the face of large fluctuations in abiotic conditions (Davy et al. 1997; Muller-Parker \& Davy 2001). On the other hand, under experimental conditions in response to extreme thermal regimes, a range of alterations in these key indicators of symbiotic state were observed. Exposure to both cold $\left(8^{\circ} \mathrm{C}\right)$ and heat $\left(28^{\circ} \mathrm{C}\right)$ treatment for a period of six weeks resulted in a significant reduction in Symbiodinium density relative to controls. Heat treatment also resulted in an increase in both chlorophyll $a$ and $c_{2}$ per symbiont cell in the heat-treated group when comparing pre- to post-treated samples, and also relative to controls at the end of the treatment period. 
While the symbiotic relationship appeared to remain stable between seasons, notable variations in a number of external environmental parameters were observed. Both the minimum and maximum daily air temperatures $\left(T_{a}\right)$ were significantly higher in the summer than the winter collection period. Additionally, levels of chlorophyll $a$, total chlorophyll and total organic carbon (TOC) were all significantly higher in seawater samples from Point Halswell in summer than winter. In contrast, nitrogen (as nitrate) levels were significantly lower in summer than winter, while dissolved reactive phosphorus and ammonium remained stable between seasons. The increase in chlorophyll $a$ and total chlorophyll are indicative of increased productivity in the harbour in summer and presumably also correlate with greater food availability for heterotrophic feeding by A. aureoradiata. These patterns of environmental variability are typical of temperate marine environments, driven by increased sunshine hours during the summer months, which lead to subsequent increases in temperature, altered mixing patterns and increased phytoplankton productivity (Levinton 1995; Valiela 1995; Ianson et al. 2001; Kaiser 2011).

While the temperature measurements reported here indicate a higher average $T_{a}$ in summer than winter, $T_{a}$ is in fact not directly coupled to an organism's actual body temperature. Factors such as radiation, conduction, evaporation and metabolism also have an impact (Tracy 1976; Bakken 1992; Buckley et al. 2013). To account for this, Sunday et al. (2014) defined a new metric, termed operative temperature $\left(T_{e}\right)$, which defines an organism's actual body temperature in any particular environment. This provides a more biophysically accurate metric of the organism's experience, and hence is more relevant in terms of the organismal response to differing thermal regimes. To put this in perspective, anemones collected from the rocky shore at Point Halswell would have likely experienced a much greater $T_{e}$ than $T_{a}$ during a low tide coinciding with noon on a sunny day, due to direct exposure to solar radiation and conduction from the surrounding rocky substrate. Therefore, it is plausible to assume that the differences in $T_{e}$ experienced by anemones collected from the rocky shore between seasons was even greater than the recorded differences in $T_{a}$.

The trends observed in the current study, in which the association between $A$. aureoradiata and its symbionts remained stable between seasons in spite of large fluctuations in environmental conditions, is typical of many temperate cnidarian- 
dinoflagellate symbioses. A comprehensive review of the differences between temperate and tropical algal-sea anemone symbiosis clearly demonstrated that the relative stability of temperate associations occurs across a broad range of species (Muller-Parker \& Davy 2001) and hence may hold as a general trend. Subsequent studies support the generality of this trend, including a number of studies on A. aureoradiata and its symbionts. For example, Gibbons (2008) recorded algal densities of just 1.7 to 2.6 times greater in summer versus winter in A. aureoradiata collected from a temperate mudflat. Further work by Howe (2013) revealed that the symbionts harboured by A. aureoradiata are able to effectively down-regulate photosystem II (PSII) by engaging a particularly efficient non-photochemical quenching (NPQ) system, ultimately protecting themselves from photodamage. The production of ROS as a result of PSII damage is a key process in triggering the bleaching response (Weis 2008), so PSII down-regulation may be one of the main mechanisms by which symbiont densities in A. aureoradiata remain stable between seasons, even when they are exposed to such large fluctuations in environmental conditions. Interestingly, Dimond et al. (2011) explored symbiont community composition in A. elegantisima over seasons and at different tidal heights. They found no significant variation in the symbiont community composition over time despite variation in environmental paramaters. They propose that "the persistence of symbioses at high latitudes is ultimately dependent on symbionts that are well adapted to the extrinsic environment."

While symbiont density remained relatively stable between seasons in the field, exposure to extreme thermal regimes in the laboratory did result in significant reductions of symbiont density. Temperature plays an important role in the bleaching response of the cnidarian-dinoflagellate symbiosis (Muscatine \& Steen 1987). While the majority of research has focussed on the role of high temperatures in driving bleaching events, low temperatures also induce bleaching in a similar fashion (Muscatine \& Steen 1987; Saxby et al. 2003; Pontasch 2014). It is now understood that temperature-induced bleaching occurs as a result of excess levels of ROS production due to increased photosynthetic rates and photosystem damage, which eventually overwhelms the symbiont and host antioxidant defence mechanisms (Weis 2008). Expulsion of the symbionts is subsequently triggered in order to avoid ROS-mediated cellular damage (Weis 2008). Viewed in this context, it seems likely that the observed reductions in symbiont density following 
exposure of $A$. aureoradiata to both cold $\left(8^{\circ} \mathrm{C}\right)$ and heat $\left(28^{\circ} \mathrm{C}\right)$ indicates that the system was stressed beyond threshold levels at these temperatures.

The fact that symbiont density was altered in the lab while it remained stable in the field may be attributed to a number of factors: (1) Temperature regimes in the lab may have been more 'extreme' than those experienced in the field during the sampling period, resulting in higher overall ROS levels and hence triggering symbiont expulsion. While absolute temperature values were similar, temperatures in the laboratory were maintained at a constant level, while temperatures in the field would have fluctuated on a daily basis. Bleaching only occurs when cellular maintenance and repair mechanisms, such as the D1 protein repair mechanism (Warner et al. 1999) and the antioxidant defence system (Lesser 1996; Franklin et al. 2004) become overwhelmed and cannot keep up with the rate of damage (Weis 2008). Hence, ROS levels as a result of extreme temperatures in the lab would have built up continually, as opposed to field conditions, in which the system could recover during periods of more moderate temperature. The fact that both chlorophyll $a$ and $c_{2}$ per symbiont cell increased in response to heat treatment indicates that the photosynthetic capacity per symbiont cell also increased. As photosynthesis produces oxygen, which then forms ROS (Lesser 2006), the increase of chlorophyll $a$ and $c_{2}$ further implies that ROS levels would have likely been elevated in the heat-treated anemones. Measurements of the oxidative state of anemones in the field on an hourly basis during extreme temperature events would help to clarify whether this is in fact the mechanism driving the observed differences in the response of symbiont density to thermal change between field and laboratory conditions. (2) The nutrient status of anemones from the field and lab may have differed, ultimately playing a role in determining symbiont density. It has been clearly demonstrated that increased nutrient status of symbionts leads to increased densities, while nutrient deficiency results in reduced densities (Muscatine $e t$ al. 1989, 1998; McAuley \& Cook 1994; Muller-Parker et al. 1996). Hence, symbionts may have been nutrient limited under laboratory conditions and nutrient sufficient in the field. Indeed, symbiont densities in the laboratory were much lower than symbiont densities recorded in the field. This mechanism may have been particularly relevant during the summer collection period, in which temperatures were close to levels that caused symbiont loss under experimental conditions, but in which food for heterotrophic feeding, which in turn results in improved symbiont nutritional status (Davy et al. 2006), was likely much more abundant. 
While the state of the symbiotic relationship was not markedly impacted by fluctuations in environmental conditions between seasons in the field, dynamic changes were observed in numerous metabolic processes. In fact, these metabolic alterations are likely some of the key mechanisms that enable the maintenance of a stable symbiosis under such variable environmental conditions. The fact that the symbiotic state was impacted by temperature treatment in the lab at temperatures similar to those recorded in the field further supports the notion that metabolic adaptations are required to maintain a stable symbiotic relationship between seasons in the field. The partial breakdown of the relationship in the laboratory experiment also enables us to explore the metabolic processes at play when temperatures do eventually exceed threshold levels in this temperate cnidarian-dinoflagellate symbiosis.

\subsection{Metabolic modifications in response to environmental change}

The metabolic modifications observed in both host and symbiont included changes in the abundance of a range of metabolites classified as either organic acids, fatty acids, amino acids or sugars, along with a number of metabolites that could not be classified into any of these groups, and for which information regarding their potential functional role was not available. Associated with these changes in individual metabolites, large fold-changes in activity level were determined for a number of metabolic pathways. The observed metabolic shifts in response to changes in environmental conditions, both in the field and under experimental conditions are discussed in the following sections with respect to their potential role in maintaining cellular homeostasis in response to environmental variability.

\subsubsection{Organic acids}

Organic acids are composed of an organic compound bound to an acidic functional group. Most often this is a carboxyl group; however, other functional groups such as thiols, enols and phenols may also serve this role. Organic acids play important functional roles in biological systems and are involved in central metabolic pathways such as glycolysis, the tricarboxycylic acid (TCA) cycle and the pentose phosphate pathway. Recent work has also demonstrated the role of organic acids in the production of co-factors and antioxidants, and as signalling molecules in the stress response (Krüger et al. 2011). While a number of organic acids were detected in both host and symbiont fractions, in the 
present study, propanedioic acid (AKA malonate) was the only organic acid identified as being important in the response of either partner to environmental change.

Increased levels of propanedioic acid in the host in response to cold treatment highlight its potential role in thermal acclimation of the cnidarian-dinoflagellate symbiosis. Propanedioic acid is a product of uracil degradation as part of the pyrimidine metabolism pathway and is subsequently used as a substrate in beta-alanine metabolism (see amino acids below). While activity levels of both of these pathways increased in the host in response to cold treatment, which should theoretically lead to decreased pools of propanedioic acid, both pathways showed reduced activity in the symbiont, theoretically leading to increased pools of propanedioic acid. As organic acids are known to be translocated from symbiont to host (Trench 1971a; b; Sutton \& Hoegh-Guldberg 1990; Vonholdt et al. 2010), symbiont-derived propanedioic acid may have driven the observed increases in host propanedioic acid in response to cold treatment. Interestingly, it has been suggested that propanedioic acid plays an essential role in the nutritional support of the endosymbiotic bacteroid in the Rhizobium-clover symbiosis, thus enhancing nitrogen fixation by the system (Kim 2002). Propanedioic acid has also been implicated in the response to oxidative stress in rats (Liu et al. 2016). Thus, propanedioic acid may play a role in supporting optimal functioning of the cnidarian-dinoflagellate symbiosis in response to reduced temperatures by maintaining positive nutritional interactions and reducing oxidative stress.

\subsubsection{Fatty acids}

Numerous changes in the pools of fatty acids in both host and symbiont were observed in response to differing thermal regimes. The impacted metabolites included one unsaturated short chain di-carbonic acid (itaconic acid), one short chain saturated fatty acid (SFA) (Isovaleric acid) and two long chain polyunsaturated fatty acids (PUFAs) (arachidonic acid and cis-5,8,11,14,17-eicosapentaenoic acid (EPA)). Thermal change also impacted the activity of metabolic pathways associated with lipid metabolism, with a particularly prominent impact on the host anemone fraction. These changes were exemplified by the pathways involved in arachidonic acid and linoleic acid metabolism, and the biosynthesis of unsaturated fatty acids. Fatty acids play important roles in cell functioning as energy sources and storage compounds, as structural components of cell membranes and in cell 
signalling processes (Tchernov et al. 2004; Díaz-Almeyda et al. 2011; Kneeland et al. 2013). Fatty acids are also known to be translocated from symbiont to host and vice versa (Papina et al. 2003; Imbs et al. 2014). The observed changes in fatty acids and associated pathways are discussed below in the context of their known functions in biological systems.

Alterations to fatty acid pools and fatty acid-associated pathways indicate altered energy production in response to thermal change. Increased levels of the tentatively identified itaconic acid, an unsaturated short chain di-carbonic acid, in the host in response to heat treatment may imply altered TCA cycle activity under these conditions. Itaconic acid is produced from cis-aconitate, an intermediate in the TCA cycle. One explanation for the increased pools of itaconic acid is that levels of its precursor, cis-aconitate, were also elevated. Given the role of cis-aconitate in the TCA cycle, elevated levels would theoretically be associated with reduced flux through this pathway (Aggio et al. 2010), which in turn would lead to reduced production of cellular energy in the form of GTP. Although no alterations to the TCA cycle were detected via PAPi analysis, this is expected given the particular derivitisation techniques employed, which perform poorly in the identification of organic acids, the major compounds of the TCA cycle (Villas-bôas et al. 2011). Furthermore, reductions in oxidative phosphorylation under the same conditions (the metabolic process that utilises products of the TCA cycle for further energy production) reinforces the view that flux through the TCA cycle was indeed reduced. Reductions in the activity of oxidative phosphorlyation itself clearly demonstrate that the production of cellular energy was reduced in the host in response to elevated temperatures (See Sugars below).

Altered patterns of lipid metabolism in the host also indicate altered energy balance in response to thermal change and may be attributed to changes in symbiont photosynthetic rates and the corresponding patterns of photosynthate translocation. Activity levels of numerous pathways associated with fatty acid metabolism, and lipid metabolism in general, were altered in response to thermal change in the host anemone fraction. These changes were exemplified by the pathways of arachidonic acid and linoleic acid metabolism, which were both lower in summer than winter, consistent with a decrease in activity in response to heat treatment and an increase in activity in response to cold treatment. Correspondingly, symbiont photosynthetic rates were reduced in response to 
cold treatment and increased in response to heat treatment. The translocation of photosynthetic products to the host likely followed a similar pattern, as symbionts transfer the majority of their photosynthetically-fixed carbon to the animal host (Muscatine 1990; Iglesias-Prieto \& Trench 1994). This altered energy availability may in turn be responsible for the altered lipid metabolism observed in the host. As lipid metabolism is tightly linked to the production of cellular energy (i.e. via the TCA cycle) the broad scale changes in anemone lipid metabolism signify major perturbations to anemone energy balance in response to thermal change.

Both arachidonic acid and linoleic acid metabolism are central to numerous cell signalling cascades in animal systems, mediating such processes as cell growth, inflammation and immunity (Moncada \& Vane 1978; Needleman et al. 1987; Samuelsson et al. 1987; Samuelsson 1991; Funk 2001). Interestingly, strong evidence exists for the role of ROS and nitric oxide (NO) mediated cell signalling cascades in the cnidarian bleaching response (Weis 2008). As the observed changes in pathway activity in the present study were associated with changes in symbiont density, it is possible that the cell signalling cascades associated with arachidonic acid and linoleic acid metabolism also play a role in the breakdown of the cnidarian-dinoflagellate symbiosis.

Altered PUFA content may be attributed to nitrogen deficiency in the symbiont in the summer months when seawater $\mathrm{NO}_{3}-\mathrm{N}$ levels were low, a state that has previously been reported in zooxanthellae isolated from the population of anemones at Point Halswell (Morar et al. 2011). In the symbiont, levels of the long chain PUFA cis-5,8,11,14,17eicosapentaenoic acid (EPA) were higher in summer than winter. This may be the result of an adaptive strategy employed by the symbionts to maintain sufficient cellular energy stores under nitrogen deprived conditions (Jiang et al. 2014). These data corroborate the findings of Hillyer et al. (2015), who detected elevated levels of multiple PUFAs in symbionts showing signs of reduced nitrogen assimilation in response to heat stress. On the other hand, these findings are in direct contrast with a range of studies that detected reductions in the relative PUFA content of tropical Symbiodinium spp. exposed to elevated temperatures (Papina et al. 2007; Díaz-Almeyda et al. 2011; Kneeland et al. 2013). As the changes in PUFA content of these tropical Symbiodinium was driven by reduced thylakoid membrane PUFA content in order to maintain membrane stability, and 
hence enhance thermal tolerance, the contrasting results observed in the present study suggest that this mechanism is not utilised, or was not activated in this temperate system.

Patterns of PUFA and SFA content in the host indicate a down regulation of metabolic activity in a manner concordant with the 'membrane pacemaker' theory of metabolism (Hulbert 2003). In the host, a large fold increase was detected in levels of the long chain PUFA arachidonic acid in summer relative to winter, while levels of the SFA isovaleric acid were elevated in response to cold treatment. The 'membrane pacemaker' theory of metabolism suggests that the degree of unsaturation of membrane FAs, in particular in mitochondria, determines metabolic rate (Hulbert 2003). Numerous marine invertebrates reduce metabolic rate in order to reduce cellular oxidative damage as a result of elevated temperatures (Abele 2011). While total lipid pools were not determined here, polar and semi-polar fatty acids constitute a major portion (Imbs \& Yakovleva 2012). Hence, elevated arachidonic acid in summer and reduced isovaleric acid in response to low temperatures suggests that $A$. aureoradiata alters the composition of membrane FAs as a mechanism to avoid oxidative damage driven by elevated temperatures. However, absolute quantification of mitochondrial membrane lipids in response to thermal stress would be required to confirm this proposition.

The observed reduction in the biosynthesis of unsaturated fatty acids in the anemone fraction in summer relative to winter appears counterintuitive given the elevated levels of arachidonic acid under these conditions. Additionally, the reduced activity of this pathway is not consistent with the 'membrane pacemaker' theory suggested above. However, the counterintuitive reductions in pathway activity may simply be an artefact of the theoretical interplay between the KEGG database and the PAPi algorithm, in that elevated levels of any metabolite recorded for a given pathway in the KEGG database contribute to a reduced activity score for that pathway, when in fact they could signify increased activity (Booth et al. 2013). In the algal fraction, elevated biosynthesis of unsaturated fatty acids in summer relative to winter is consistent with the findings of elevated EPA concentrations under these conditions. Additionally, these findings invoke the possibility that symbionts up-regulate oxygen-consuming desaturase systems as a mechanism to reduce heat-induced oxidative damage (Guerzoni et al. 2001). 


\subsubsection{Amino acids}

Amino acids constitute the building blocks of proteins required for cell growth and homeostatic functioning. They may also be catabolised as sources of energy and nitrogen. Furthermore, amino acids may act as antioxidants and osmoprotectants and are precursors to the production of a wide range of alkaloids. In the cnidarian-dinoflagellate symbiosis nitrogen is mostly assimilated by the algal symbiont and nitrogen containing amino acids are subsequently translocated to the host (Wang \& Douglas 1999). In the present study, alterations to free amino acid pools and amino acid associated metabolic pathways were consistent with modifications to key homeostatic processes in response to thermal change.

The aromatic amino acid tryptophan is a precursor to the production of numerous alkaloids (Dixon 2001). In the present study, tryptophan metabolism increased significantly in host anemones in response to cold treatment, while the concentration of tryptamine (produced in the first step of tryptophan metabolism by the action of aromaticL-amino-acid decarboxylase on tryptophan) was significantly greater in the host in summer compared to winter. Boutilier (2012) demonstrated that in the SymbiodiniumZoanthus symbiosis tryptophan is synthesised by the symbiont and subsequently translocated to the host. Hence, the altered tryptophan metabolism and tryptamine levels in the host may indicate reduced production of symbiont tryptophan in winter/cold conditions and increased production of symbiont tryptophan in summer/hot conditions. Associated with these changes in tryptophan metabolism, alkaloid biosynthetic pathways in the symbiont, including indole alkaloid biosynthesis; the biosynthesis of alkaloids derived from the shikimate pathway; tropane, piperidine and pyridine alkaloid biosynthesis and the biosynthesis of alkaloids derived from ornithine, lysine and nicotinic acid, showed a general trend towards increased activity in summer compared to winter, consistent with an increase in activity in response to heat treatment. While little is known of the role of alkaloids in the cnidarian-dinoflagellate symbiosis, a C30 alakaloid, zooxanthellamine, has been identified in cultured Symbiodinium by Nakamura et al. (1998). Interestingly, Kaplan et al. (2004) have suggested that in Arabidopsis, increased levels of branched chain amino acids in response to temperature stress are used to support the production of secondary metabolites to defend a stress weakened plant against pathogen attack. The data presented here corroborate the suggestions of Kaplan et al. (2004), and extend the model to the role of aromatic amino acids in a new model 
organism, suggesting that tryptophan production may be elevated in Symbiodinium in response to temperature stress to support the production of alkaloids for pathogen defence. The data also suggests that Symbiodinium may be capable of synthesising not only one, but a wide array of alkaloids.

Patterns of the activity of pyrimidine metabolism and the metabolism of downstream products suggests these processes played a central role in the energetic homeostasis of both partners of the symbiosis in response to thermal change. Large and significant reductions in the activity of pyrimidine metabolism and $\beta$-alanine metabolism were detected in the symbiont in response to cold treatment, while the activity of both of these pathways increased in response to heat treatment. In the host, the activity of both pathways was lower in summer compared to winter, consistent with a trend towards decreased activity in response to heat treatment and increased activity in response to cold treatment. Pyrimidine metabolism involves the degradation of uracil, from RNA, and thymine, from DNA, to $\beta$-alanine and $\beta$-aminoisobutyric acid, respectively (Van Kuilenburg et al. 2004). Consistent with the observed patterns of metabolic pathway activity, levels of $\beta$-aminoisobutyric acid increased in the host in response to heat treatment while $\beta$-Alanine concentration increased significantly in the symbiont in response to cold treatment and decreased significantly in response to heat treatment. As $\beta$ aminoisobutyric acid is normally catabolised to intermediates of the TCA cycle (Pollitt et al. 1985; Van Kuilenburg et al. 2004) these results indicate reduced $\beta$-aminoisobutyric acid degradation and the subsequent availability of its products to the TCA cycle. Of particular relevance, $\beta$-alanine is a precursor to the pantothenate moiety of coenzyme $\mathrm{A}$ (CoA) and acyl carrier protein (ACP) (Fouad 2004). A large fold increase was detected in the activity of pantothenate and CoA biosynthesis in the host in response to cold treatment and a large fold decrease was detected in response to heat treatment. Taken together, these findings suggest that the production of CoA and ACP may have been down regulated in the anemone host in response to high temperatures and up regulated in response to low temperatures, with opposite patterns occurring in the algal symbiont. One of the major roles of $\mathrm{CoA}$ is the transport of fatty acids from the cytoplasm to the mitochondria where they are oxidised, producing acetyl CoA for use in the TCA cycle (Leonardi et al. 2005). Another product of $\beta$-alanine metabolism, propanoyl-CoA, is the primary substrate of propanoate metabolism, which produces succinyl-CoA, an intermediate of the TCA cycle. Propanoate metabolism was up regulated in the host in response to cold treatment. The 
observed patterns in the activity of each of the above pathways and their substrates points towards reduced energy production via the TCA cycle in the host in summer/hot conditions and increased energy production in winter/cold conditions, with opposite patterns occurring in the algal symbiont.

A number of metabolic pathways associated with the direct biosynthesis or degradation of proteinogenic amino acids, along with specific substrates of these pathways, were seasonally variable and responded accordingly to both cold and heat treatment. Arginine and proline metabolism increased in the symbiont, but decreased in the host, in response to heat treatment. This indicates an increase in symbiont and reduction in host deamination and respiratory use of amino acids in response to heat treatment (Wang \& Douglas 1998). In the host this may driven by an increased availability of symbiont photosynthate in summer/hot conditions and reduced availability of symbiont photosynthate in winter/cold conditions, consistent with the observed changes in symbiont photosynthetic capacity between these conditions. The concentration of cis-4hydroxy-D-proline, produced by the action of prolyl 4-hydroxylase on L-proline and 2oxogluatarate (a substrate of the TCA cycle), was significantly higher in the host in summer compared to winter. Interestingly, cis-4-hydroxy-D-proline is involved in hypoxia inducible factor 1 (HIF-1) signalling cascades. In the symbionts, the activity of the HIF-1 signaling pathway was greater in summer compared to winter, consistent with increased activity in response to heat treatment and reduced activity in response to cold treatment, suggesting symbionts may have been oxygen limited under these conditions and altered oxygen associated processes via HIF-1 pathways in order to maintain metabolic homeostasis (Gorr et al. 2006). The concentration of another substrate of arginine and proline metabolism, the polyamine putrescine, showed a large fold decrease in the symbiont in response to both cold and heat treatment. 1,4-Butandiamine (AKA putrescine) is produced from ornithine via the action of ornithine carboxylase, which has been previously identified in Symbiodinium sp., and may indicate alterations to the process of cell growth and proliferation (Streamer 1980; Jänne et al. 2004, 2005). The activity of glycine, serine and threonine metabolism was lower in the host in summer compared to winter, consistent with reduced activity in response to heat treatment and increased activity in response to cold treatment. These findings further support altered respiratory use of amino acids by the host in response to altered patterns of photosynthate translocation (Wang \& Douglas 1998). The only substrate from this pathway to be 
significantly impacted was N,N dimethylglycine, a derivative of glycine, which had a significantly greater concentration in the host in summer compared to winter. The activity of the lysine degradation pathway decreased in the symbiont in response to cold treatment and was lower in the host in summer compared to winter, indicating a reduction in the supply of acetyl-CoA to the TCA cycle. On the other hand, the activity of lysine biosynthesis decreased in the host in response to heat treatment, signifying either a reduced demand or reduced availability of 2-oxogluterate from the TCA cycle and acetylCoA from glycolysis, normally required for this process. Again, both of these findings indicate altered host respiratory use of amino acids in response to photosynthate availability (Wang \& Douglas 1998). Consistent with the observed patterns of pathway activity, L-lysine itself showed a large fold decrease in the host in response to cold treatment. L-lysine is 'essential' to the host in the Symbiodinium-zooanthus association (Boutilier 2012). Hence, the observed changes in L-lysine may be attributed to reduced translocation of L-lysine from the symbiont, which seems likely given the reduced photosynthetic capacity of the symbionts under these conditions. Overall, these findings add further weight to the previously discussed data, suggesting that altered energy metabolism is a core feature of the metabolic response of both partners of the cnidariandinoflagellate symbiosis to thermal change, and further implies that these pathways, along with symbiont photosynthetic capacity, are particularly important in this process.

L-Lysine is also a substrate of biotin metabolism, in particular, as a product of the cleavage of biocytin by biotidinase to L-lysine and biotin, releasing free biotin for use in cellular metabolism (Hymes \& Wolf 1996). Given the role of L-lysine in biotin metabolism, the reduced activity of biotin metabolism detected in the host in response to heat treatment and increased activity in response to cold treatment, likely indicates reduced and increased levels of free biotin in the host in response to cold and heat treatment, respectively. Biotin is an essential vitamin in animals, but can be synthesised from fatty acids in plants. The role of biotin is as a cofactor to key enzymes of gluconeogenesis, fatty acid metabolism and amino acid catabolism (Pacheco-Alvarez et al. 2002). Taken with the results presented here, it appears that biotin levels were elevated in the host in response to cold treatment to satisfy the demand required for elevated rates of gluconeogenesis, fatty acid metabolism and amino acid catabolism, while demand was reduced for these processes in the host in response to heat treatment. This suggestion is consistent with the interpretations of fatty acid and amino acid metabolism previously 
discussed, and implicates biotin as a crucial factor in the maintenance of metabolic homeostasis in cnidaria in response to thermal change.

\subsubsection{Sugars}

Numerous metabolic pathways associated with the production of sugars and the metabolism of sugars to release cellular energy (ATP) were variable between seasons and responded to thermal change in both the symbiont and host. Significant changes in the abundance of several key sugars and sugar derivatives were also detected. Sugars are produced via photosynthesis and constitute the primary energy source and storage compound of Symbiodinium. Additionally, sugars can be translocated from symbiont to host, acting as a primary source of energy rich nutrition.

A number of sugar derivatives that could not be confidently associated with any identified metabolic pathways were impacted by thermal change. The changes in these metabolites suggests they are important in the response to thermal change of either symbiont or host (as indicated) of the Anthopleura-Symbiodinium association. Elevated levels of a sugar acid identified as either erythronic or threonic acid in the host in response to heat treatment suggests that protein glycation or L-ascorbate (vitamin C) degradation may have increased under these conditions (Harding et al. 1999). A reduction in levels of the glycoside methyl galactoside in summer compared to winter in the symbiont may be attributed to the role of glycosides as plant secondary metabolites, including as defence and aroma compounds (Ketudat Cairns et al. 2015). Glycosides also play a role in the recovery of Arabidopsis thaliana from numerous abiotic stressors, including temperature (Roepke \& Bozzo 2015), further supporting the notion that methyl galactoside may have been involved in maintaining organismal functioning in the symbiont in response to thermal change between seasons. Another glycoside, identified as methyl beta-Dglucopyranoside displayed a large and significant decrease in host anemones as a result of cold treatment. In contrast, a large and significant increase was detected in the host as a result of heat treatment. Methyl beta-D-glucopyranoside was recently identified in the fruit juice of Carica papaya and was shown to have powerful antioxidant properties (Galang et al. 2016). Hence, host anemones may have been under oxidative stress as a result of heat treatment, resulting in the active accumulation of methyl beta-D- 
glucopyranoside as a protective mechanism against oxidative damage. Overall these findings suggest that while the role of glycosides and sugar acids in metabolism are not well understood, they may be important factors in the metabolic response of the cnidarian-dinoflagellate symbiosis to thermal change.

In the symbiont, patterns of central carbon metabolism and sugar metabolism, both between seasons and in response to thermal change, indicate that sugar production and metabolic rate was reduced in response to low temperatures and elevated in response to high temperatures. In Symbiodinium, adenosine triphosphate (ATP) and reduced nicotinamide adenine dinucleotide phosphate (NADPH) are produced by photosynthesis ("light dependent reactions") and used by the Calvin cycle ("light independent reactions") to fix carbon, thereby producing glyceraldehyde-3-phosphate (G3P) (Trench 1993). G3P is then utilised in glycolysis/gluconeogenesis and additional downstream pathways, leading to the production of energy rich sugar molecules, or contributing to the generation of cellular energy. Hence, photosynthesis is a key driver of the production of energy rich sugar molecules and the availability of cellular energy in photosynthetic organisms. Indeed, in the present study, symbiont photosynthetic capacity was reduced in winter/cold conditions and elevated in summer/hot conditions, while a generally equivalent pattern was observed in the activity of the energy generating pathways of central carbon metabolism, including the Calvin cycle, glycolysis, the pentose phosphate pathway and oxidative phosphorylation; along with pathways responsible for the production of sugars, including starch and sucrose metabolism, fructose and mannose metabolism, galactose metabolism and amino sugar and nucleotide sugar metabolism (Figure 4.1).

Additionally, certain substrates involved in these pathways were identified as key metabolites in the symbiont response to thermal change. Consistent with the activity of amino sugar and nucleotide sugar metabolism, galactose metabolism and fructose and mannose metabolism, the sugar tentatively identified as alpha D-mannopyranose, one of the four ring configurations in which mannose is commonly found, showed a large fold decrease as a result of heat treatment in the symbiont. Also associated with fructose and mannose metabolism, higher levels of mannitol in the symbiont in winter may be attributed to its known role as an antioxidant preventing lipid peroxidation during cold stress in plants (Smirnoff \& Cumbes 1989). Indeed, elevated levels of ROS have been recorded in Symbiodinium exposed to cold stress (Pontasch 2014). Hence, if cellular 
homeostasis is to be maintained, a mechanism would be required to deactivate this ROS. Maltose and sucrose, both substrates of starch and sucrose metabolism, were identified as important metabolites in distinguishing symbiont metabolite profiles between conditions. Sucrose also showed a large fold decrease in the symbiont in response to heat treatment. These results suggest that the degradation of starch to sucrose, and its subsequent use for the generation of cellular energy via glycolysis, was elevated in response to heat treatment. Starch mobilisation leading to increased levels of soluble sugars and contributing to a profound re-modelling of central carbohydrate metabolism has been recorded in Arabidopsis in response to short term heat stress (Kaplan et al. 2004; Guy et al. 2008). Hence, a similar mechanism may be involved in the symbiont response to thermal change, the major difference being that sucrose did not accumulate. Perhaps it was not required in the role of compatible solute as has been suggested for Arabidopsis (Kaplan et al. 2004). If so, sucrose may have instead simply contributed to the production of cellular energy. The accumulation of beta-D-glucose in the symbiont in response to cold treatment, along with its status based on PCA as an important metabolite in distinguishing cold and heat treated symbionts from controls, can likely be attributed to the overall reduced flux through central carbon and sugar metabolic pathways (discussed above) of which beta-D-glucose is a key substrate. These findings are consistent with elevated levels of glucose observed in Arabidopsis in response to low temperatures, which have been attributed to the role of glucose as a compatible solute (Kaplan et al. 2004; Guy et al. 2008). As glucose is potentially the most abundant metabolite transferred from symbiont to host (Burriesci et al. 2012), elevated pools of glucose in the symbiont may also indicate reduced availability of this important metabolite to the host anemone. Ribose, a product of the pentose phosphate pathway, was identified via PCA as an important metabolite in distinguishing between both cold and heat treated symbionts relative to controls. Along with the patterns of mannose, maltose, sucrose and glucose abundance, this finding further strengthens the view that as in Arapadopsis (Guy et al. 2008), alterations to central carbohydrate metabolism are a core feature of the metabolic response of Symbiodinium to thermal change.

In contrast to the symbiont, patterns of central carbon and sugar metabolism in the host indicate that the metabolism of sugars for the production of cellular energy was elevated in response to low temperatures and reduced in response to high temperatures. Peptidoglycan biosynthesis was lower in the host in summer compared to winter, 
consistent with reduced activity in response to heat treatment and increased activity in response to cold treatment. As peptidoglycans are potential energy sources translocated from the host to symbiont (Logan et al. 2010), this suggests a reduced use of these molecules by the host for energy generation under these conditions. The activity of starch and sucrose metabolism was lower in the host in summer compared to winter. Sucrose also showed a large fold decrease in the host cold treated group, while a large fold increase was observed in the host heat treated group. As sucrose is used in the generation of energy via glycolysis, this again indicates reduced use of sugars for the generation of cellular energy in response to heat treatment and increased use of sugars for the generation of cellular energy in response to cold treatment. This is further supported by the observed reduction in activity of fructose and mannose metabolism in response to cold treatment and the reduced activity of amino sugar and nucleotide sugar metabolism in the host in summer relative to winter. Finally, the activity of oxidative phosphorylation was reduced in the host in summer/hot conditions and elevated in winter/cold conditions. Oxidative phosphorylation comprises the final step of central carbon metabolism, which produces the majority of aerobically generated cellular energy in the form of ATP. These findings draw together the patterns of pathway activity observed in organic acid, fatty acid, amino acid, and sugar metabolism, which all showed patterns suggesting reduced host metabolic rate in hot/summer and increased hot metabolic rate in winter/cold conditions.

\subsubsection{Compounds of unknown function}

A number of compounds were identified in both the symbiont (Table A 2.1) and host (Table A 2.2), which were not registered in the KEGG database and for which no information regarding their function was available in the major chemical database PubChem. Hence, these did not have any impact on the calculation of metabolic pathway activity via PAPi analysis and their metabolic function remains either unknown or obscure. However, these compounds were identified with the same degree of confidence as all other metabolites. Hence, further research into their potential role in the cnidariandinoflagellate symbiosis is warranted. 


\subsection{Proposed model of metabolic pathway response to thermal change in the $A$. aureoradiata-Symbiodinium symbiosis}

In general, most metabolic pathways were up regulated in the host in response to cold treatment and down regulated in response to heat treatment (Figure 4.1). The exception being fructose and mannose metabolism, for which reduced activity was detected in response to cold treatment. Based on the particular pathways impacted, these findings suggest a wide scale reduction in energy metabolism in the host in response to heat treatment. This is similar to the processes employed by other marine invertebrates, which reduce mitochondrial energy production in order to avoid oxidative damage (Abele 2011). In the symbiont, patterns of pathway activity were opposite to that observed in the host, with a general increase in response to heat treatment and reduction in response to heat treatment (Figure 4.1). Consistent with these findings, symbiont photosynthetic capacity was also elevated in response to heat treatment and reduced in response to cold treatment. Hence, the availability of energy as a function of photosynthetic rate may have been a key factor in driving the observed alterations to metabolic pathway activity in the symbiont. While this work provides initial insight on the metabolic functioning of a temperate cnidarian-dinoflagellate symbiosis, further work employing a combination of both metabolomics and transcriptomics would help to more clearly elucidate the impact of thermal change on metabolic processes in this symbiotic association. 
Figure 4.1 Metabolic pathways and their response to differing thermal regimes in the Anthopleura-Symbiodinium symbiosis. (A) Under control conditions; (B) in response to cold/winter; and (C) in response to heat/summer. Black outlines indicate no data or that the magnitude of change was very small; red outlines indicate a large ( $\geq 2$ fold) decrease in pathway activity; green outlines indicate a large ( $\geq 2$ fold) increase in pathway activity. Green cell $=$ chloroplasts, red cell $=$ mitochondria, brown cell $=$ symbiont, beige cell $=$ host. Amino acid metabolism, lipid metabolism and nucleotide metabolism represent sets composed of numerous metabolic pathways. An indicated change to one of these higher level groups simply implies that one or more of the pathways within that group changed. A number of pathways that showed large fold changes are not depicted, including: glycosaminoglycan biosynthesis, pantothenate and CoA biosynthesis and biotin metabolism in the host and the HIF-1 signaling pathway and numerous alkaloid biosynthetic pathways in the symbiont. 

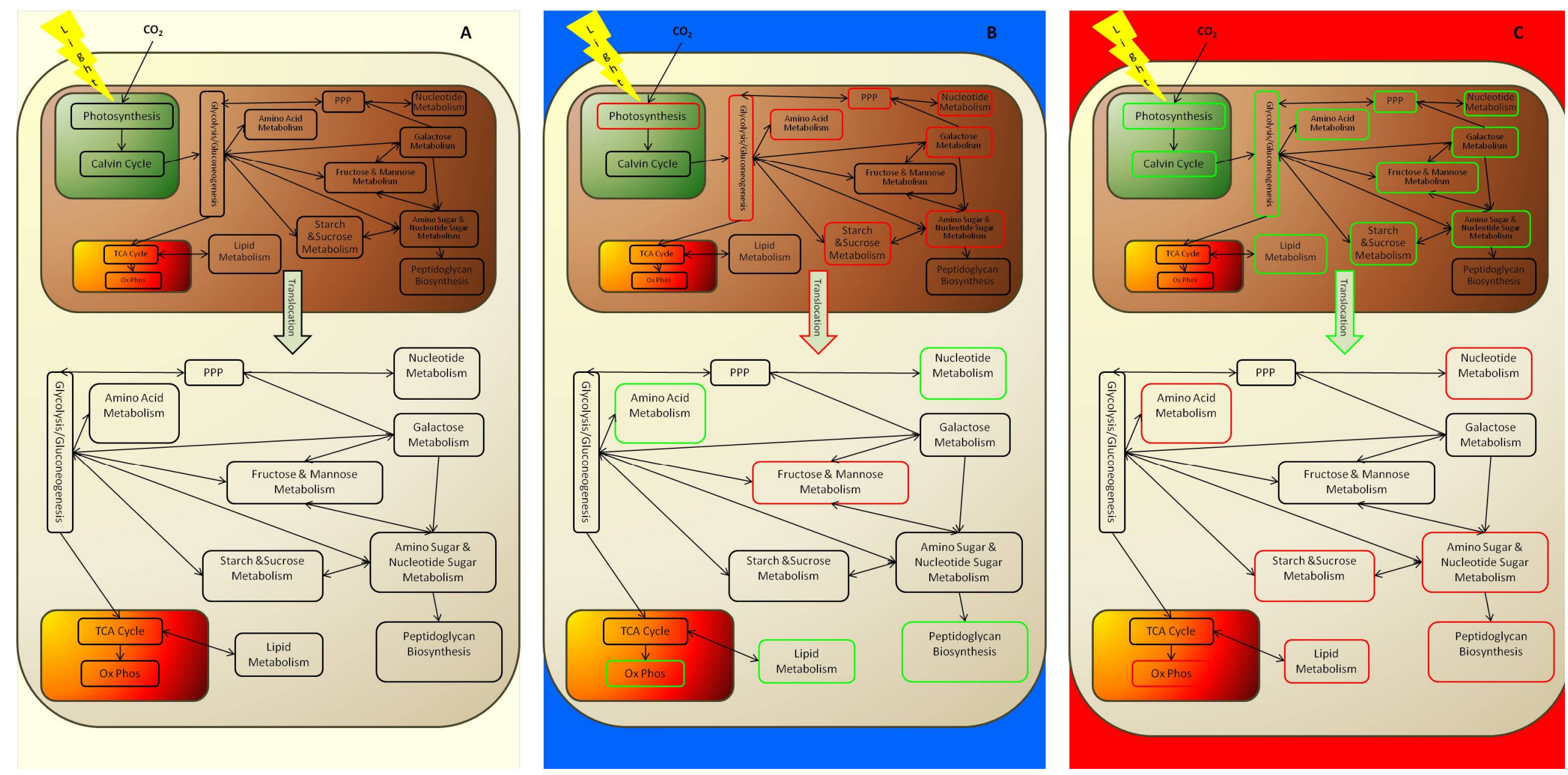


\subsection{Conclusion}

The application of GC-MS and pathway activity profiling to explore the metabolic functioning of a temperate cnidarian-dinoflagellate symbiosis has provided new insight on how these organisms respond to thermal stress. It appears that the host anemones metabolic rate may not be directly correlated with symbiont photosynthetic rate as has been previously described (Harland \& Davies 1995). Instead, in this temperate system the host appears to down regulate metabolic activity in response to elevated temperatures, perhaps as a mechanism to avoid oxidative damage, as is seen in other marine invertebrates (Abele 2011). As the cnidarian 'bleaching' response is known to be triggered, at least partially, by oxidative damage to the host (Weis 2008) the ability of $A$. aureoradiata to down regulate metabolic activity in response to heat stress may constitute a primary mechanism by which a stable relationship can be maintained under such highly variable environmental conditions. This work provides a benchmark against which the metabolic functioning of tropical cnidarian-dinoflagellate symbioses can be compared in future studies. This will help to further clarify the picture regarding the mechanisms that contribute to the relative stability of temperate associations in comparison to the sensitivity of tropical associations. In the face of global climate change threatening many marine ecosystems, and in particular coral reefs, developing our understanding of these processes is imperative if we are to ensure their longevity. In this light, metabolomics studies provide a powerful tool to understand these processes and hence inform appropriate management of these ecologically important organisms. 


\section{APPENDICES}

\section{Appendix 1}

Table A 1.1 List of full names matched to names that were concatenated in graphs depicting the results of univariate analysis

\begin{tabular}{|c|c|}
\hline Concatenated name & Full name \\
\hline \multicolumn{2}{|c|}{ Host seasonal comparison } \\
\hline Cetyl alcohol & $\begin{array}{l}\text { Cetyl alcohol or Silane, }(1,1- \\
\text { dimethylethyl)(hexadecyloxy)dimethyl- }\end{array}$ \\
\hline L-Norleucine, N propoxycarbonyl & $\begin{array}{l}\text { L-Norleucine, N-propoxycarbonyl-, nonyl ester } \\
\text { or L-Norvaline, N-butoxycarbonyl-, tetradecyl } \\
\text { ester or N-Methyl-DL-leucine }\end{array}$ \\
\hline \multicolumn{2}{|c|}{ Symbiont seasonal comparison } \\
\hline 4-[2-(4-hydroxy-3-methyl & $\begin{array}{l}\text { 4-[2-(4-hydroxy-3-methylphenyl)propan-2-yl]- } \\
\text { 2-methylphenol }\end{array}$ \\
\hline Dimethylethylamine & $\begin{array}{l}\text { Dimethylethylamine or Carbonic acid, 2- } \\
\text { dimethylaminoethyl isobutyl ester }\end{array}$ \\
\hline \multicolumn{2}{|c|}{ Host cold treatment } \\
\hline 2-Hydroxybut-2-enoic acid & $\begin{array}{l}\text { 2-Hydroxybut-2-enoic acid or Trimethylsilyl-3- } \\
\text { [(trimethylsilyl)oxy]but-2-enoate }\end{array}$ \\
\hline Alanine, N-methyl-N-butoxycarbonyl & $\begin{array}{l}\text { Alanine, N-methyl-N-butoxycarbonyl-, } \\
\text { isohexyl ester }\end{array}$ \\
\hline
\end{tabular}




\begin{tabular}{|c|c|}
\hline alpha-D-Glucopyranoside, methyl & $\begin{array}{l}\text { alpha-D-Glucopyranoside, methyl 2- } \\
\text { (acetylamino)-2-deoxy-3-O-(trimethylsilyl)-, } \\
\text { cyclic methylboronate }\end{array}$ \\
\hline alpha-D-Mannopyranose & $\begin{array}{l}\text { alpha-D-Mannopyranose or alpha-D- } \\
\text { Galactopyranose or beta-D-Glucopyranose }\end{array}$ \\
\hline $\mathrm{N}-[(\mathrm{E})-2,2-$ Dimethylpropylidene & $\begin{array}{l}\mathrm{N}-[(\mathrm{E})-2,2- \\
\text { Dimethylpropylidene](trimethyl)silanamine or } \\
\text { N-Ethylformamide }\end{array}$ \\
\hline \multicolumn{2}{|c|}{ Host heat treatment } \\
\hline 1-Piperidineethanol & $\begin{array}{l}\text { 1-Piperidineethanol or 2-Pyrrolidone-5- } \\
\text { carboxylic acid, N-methyl, methyl ester or 4,4- } \\
\text { Dimethoxybutyronitrile }\end{array}$ \\
\hline 2-Ethylnaphtho[1,8-de] & 2-Ethylnaphtho[1,8-de][1,3,2]dioxaborinine \\
\hline 2-O-Glycerol-alpha-D-galactopyranoside & $\begin{array}{l}\text { 2-O-Glycerol-alpha-D-galactopyranoside, } \\
\text { hexa-TMS }\end{array}$ \\
\hline Phosphoric acid, bis(trimethylsilyl) & $\begin{array}{l}\text { Phosphoric acid, bis(trimethylsilyl)monomethyl } \\
\text { ester }\end{array}$ \\
\hline \multicolumn{2}{|c|}{ Symbiont cold treatment } \\
\hline alpha-D-Mannopyranose & $\begin{array}{l}\text { alpha-D-Mannopyranose or alpha-D- } \\
\text { Galactopyranose or beta-D-Glucopyranose }\end{array}$ \\
\hline beta-D-Glucopyranose & $\begin{array}{l}\text { alpha-D-Mannopyranose or alpha-D- } \\
\text { Galactopyranose or beta-D-Glucopyranose }\end{array}$ \\
\hline beta-l-Galactopyranoside, methyl & $\begin{array}{l}\text { beta-1-Galactopyranoside, methyl 6-deoxy- } \\
\text { 2,3,4-tris-O-(trimethylsilyl)- }\end{array}$ \\
\hline Dimethylethylamine & $\begin{array}{l}\text { Dimethylethylamine or Carbonic acid, 2- } \\
\text { dimethylaminoethyl isobutyl ester }\end{array}$ \\
\hline
\end{tabular}




\begin{tabular}{|l|l|}
\hline d-Galactose (1Z) & $\begin{array}{l}\text { d-Galactose, 2,3,4,5,6-pentakis-O- } \\
\text { (trimethylsilyl)-, o-methyloxyme, (1Z)- or d- } \\
\text { Mannose, 2,3,4,5,6-pentakis-O-(trimethylsilyl)- } \\
\text { o-methyloxyme, (1Z)- or or Galactose oxime } \\
\text { hexakis(trimethylsilyl) }\end{array}$ \\
\hline Pentyl 2-(2-methoxy & $\begin{array}{l}\text { pentyl 2-(2- } \\
\text { methoxyethoxycarbonylamino)pentanoate }\end{array}$ \\
\hline Symbiont heat treatment
\end{tabular}




\section{Appendix 2}

Table A 2.1 Compounds with an unknown function or classification that were identified as being important via fold-change analysis, univariate (t-tests) or multivariate (PCA) analysis in the symbiont. $\mathrm{Y}=\mathrm{Yes}$, identified as important.

\begin{tabular}{|c|c|c|c|c|c|c|c|c|c|}
\hline \multirow[t]{2}{*}{ Compound } & \multicolumn{3}{|c|}{ Summer vs. winter } & \multicolumn{3}{|c|}{ Cold } & \multicolumn{3}{|c|}{ Hot } \\
\hline & FC & PCA & Sig. & FC & PCA & Sig. & FC & PCA & Sig. \\
\hline Amphetamine & 0.419 & & 0.004 & 2.788 & $\mathrm{Y}$ & & & & \\
\hline $\begin{array}{l}\text { beta-l- } \\
\text { galactopyranoside, } \\
\text { methyl 6-deoxy-2,3,4- } \\
\text { tris-O-(trimethylsilyl) }\end{array}$ & & & & & & & 0.435 & & 0.032 \\
\hline beta-D-galactofuranose & & $\mathrm{Y}$ & & & $\mathrm{Y}$ & & & $\mathrm{Y}$ & \\
\hline $\begin{array}{l}\text { 1-isopropenyl-2,3- } \\
\text { dimethylcyclopentane }\end{array}$ & Down & & 0.038 & & & & & & \\
\hline $\begin{array}{l}\text { 11,11-dimethyl- } \\
\text { spiro[2,9]dodeca-3,7- } \\
\text { dien }\end{array}$ & Down & & 0.050 & & & & & & \\
\hline $\begin{array}{l}\text { alanine, N-methyl-n- } \\
\text { butoxycarbonyl-, } \\
\text { isohexyl ester }\end{array}$ & & $\mathrm{Y}$ & & & & & 0.347 & $\mathrm{Y}$ & \\
\hline Cyclopentanol & & & & Up & & 0.019 & & & \\
\hline $\begin{array}{l}\text { pentyl 2-(2- } \\
\text { methoxyethoxycarbonyl } \\
\text { amino) pentanoate }\end{array}$ & & & & 2.597 & Y & & & $\mathrm{Y}$ & \\
\hline
\end{tabular}


Table A 2.2 Compounds with an unknown function or classification that were identified as being important via fold-change analysis, univariate (t-tests) or multivariate (PCA) analysis in the host. $\mathrm{Y}=$ Yes, identified as important.

\begin{tabular}{|c|c|c|c|c|c|c|c|c|c|}
\hline \multirow[t]{2}{*}{ Compound } & \multicolumn{3}{|c|}{ Summer vs. winter } & \multicolumn{3}{|c|}{ Cold } & \multicolumn{3}{|c|}{ Hot } \\
\hline & FC & PCA & Sig. & FC & PCA & Sig. & FC & PCA & Sig. \\
\hline $\begin{array}{l}\text { Unknown inositol } \\
\text { stereoisomer }\end{array}$ & & & & & & & & & 0.005 \\
\hline 4-Hydroxypyridine & 2.386 & $\mathrm{~N}$ & $\mathrm{~N}$ & 2.332 & $\mathrm{Y}$ & $\mathrm{N}$ & Up & $\mathrm{Y}$ & $\mathrm{N}$ \\
\hline 2-ethylquinoline & 2.350 & $\mathrm{Y}$ & $\mathrm{N}$ & $\mathrm{N}$ & $\mathrm{Y}$ & $\mathrm{N}$ & Up & $\mathrm{N}$ & $\mathrm{N}$ \\
\hline Conhydrine & 2.016 & & & & & & 2.394 & & \\
\hline $\begin{array}{l}\text { L-Alanine, } \mathrm{N} \text { - } \\
\text { ethoxycarbonyl-, decyl } \\
\text { ester }\end{array}$ & 2.006 & & & 2.732 & & & 3.125 & & \\
\hline 2-butanol & & & & & & 0.030 & & & \\
\hline 3-methoxybutan-2-ol & & & & & & 0.037 & & & \\
\hline $\begin{array}{l}\text { 3,3'-dinonyl-2,2'- } \\
\text { bithiazolidine }\end{array}$ & & & & 4.261 & $\mathrm{Y}$ & & & $\mathrm{Y}$ & \\
\hline $\begin{array}{l}\mathrm{N}-[(\mathrm{E})-2,2- \\
\text { dimethylpropylidene](tr } \\
\text { imethyl)silanamine or } \\
\text { N-ethylformamide }\end{array}$ & & & & & & 0.040 & & & \\
\hline $\begin{array}{l}\text { 2-(2-Hydroxy-1- } \\
\text { methylethyl)-1H- } \\
\text { isoindole-1,3(2H)-dione }\end{array}$ & & & & 2.830 & $\mathrm{Y}$ & & & $\mathrm{Y}$ & \\
\hline Oleamide & Up & & & & & & & & \\
\hline Ethylene glycol & & & & 2.125 & & & 2.020 & & \\
\hline
\end{tabular}




\begin{tabular}{|l|l|l|l|l|l|l|l|l|l|l|}
\hline Compound & \multicolumn{2}{|c|}{ Summer vs. winter } & \multicolumn{3}{c|}{ Cold } & \multicolumn{3}{c|}{ Hot } \\
\hline & FC & PCA & Sig. & FC & PCA & Sig. & FC & PCA & Sig. \\
\hline 5-Androsten-3-beta-ol & & & & & Y & & & Y & \\
\hline Amphetamine & & & & & & & & & 0.038 \\
\hline Benzeneacetic acid & & & & & & & & & 0.021 \\
\hline Ethylamine & & & & & & & & & 0.033 \\
\hline $\begin{array}{l}\text { Methyl isothiocyanate } \\
\text { 2-Ethylnaphtho[1,8- } \\
\text { de][1,3,2]dioxaborinine }\end{array}$ & & & & & & & & & 0.046 \\
\hline
\end{tabular}




\section{REFERENCES}

Abele D (2011) Temperature Adaptation in a Changing Climate: Marine Fish and Invertebrates. In: Temperature Adaptation in a Changing Climate: Nature at Risk (eds Storey K., Tanino K.), pp. 67-79.

Aggio RBM, Ruggiero K, Villas-Bôas SG (2010) Pathway Activity Profiling (PAPi): from the metabolite profile to the metabolic pathway activity. Bioinformatics (Oxford, England), 26, 2969-76.

Babcock RC, Bull GD, Harrison PL et al. (1986) Synchronous spawnings of 105 scleractinian coral species on the Great Barrier Reef. Marine Biology, 90, 379-394.

Badgley B, Lipschultz F, Sebens K (2006) Nitrate uptake by the reef coral Diploria strigosa: effects of concentration, water flow, and irradiance. Marine Biology, 149, 327-338.

Baeza JA (2007) The origins of symbiosis as a lifestyle in marine crabs (genus Petrolisthes) from the eastern Pacific: Does interspecific competition play a role? Revista de Biología Marina y Oceanografía, 42, 7-21.

Bago B, Becard G (2002) Bases of the obligate biotrophy of arbuscular mycorrhizal fungi. In: Mycorrhizal technology in agriculture: from genes to bioproducts (eds Gianinazzi S, Schüepp H, Barea JM, Haselwandter K), pp. 33-48. Birkhauser Verlag AG, Basel, Switzerland.

Baker AC (2003) Flexibility and specifity in coral-algal symbiosis: diversity, ecology, and biogeography of Symbiodinium. Annual Review of Ecology, Evolution, and Systematics, 34, 661-689.

Bakken GS. (1992) Measurement and Application of Operative and Standard Operative Temperatures in Ecology. American Zoologist, 32, 194-216.

Banaszak A, LaJeunesse T, Trench R (2000) The synthesis of mycosporine-like amino acids (MAAs) by cultured, symbiotic dinoflagellates. Journal of experimental marine biology and ecology, 249, 219-233.

de Bary A (1879) Die Erscheinung der Symbiose. Karl J. Trübner, Strassburg, Germany.

Berruti A, Lumini E, Balestrini R, Bianciotto V (2016) Arbuscular Mycorrhizal Fungi as Natural Biofertilizers: Let's Benefit from Past Successes. Frontiers in microbiology, 6, 1559 . 
Blank RJ, Trench RK (1985) Speciation and symbiotic dinoflagellates. Science (New York, N.Y.), 229, 656-8.

Booth SC, Weljie AM, Turner RJ (2013) Computational tools for the secondary analysis of metabolomics experiments. Computational and structural biotechnology journal, 4, e201301003.

Boutilier RM (2012) Aspects of Nitrogen Metabolism in Symbiotic Cnidarians. The University of Guelph.

Bryla DR, Eissenstat DM (2005) Respiratory costs of mycorrhizal associations. In: Plant Respiration Advances in Photosynthesis and Respiration. (eds Lambers H, RibasCarbo M), pp. 207-224. Springer-Verlag, Berlin/Heidelberg.

Buckley LB, Miller EF, Kingsolver JG (2013) Ectotherm thermal stress and specialization across altitude and latitude. Integrative and comparative biology, 53, 571-81.

Buddemeier RW, Fautin DG (1996) Saturation state and the evolution and biogeography of symbiotic calcification. Bulletin de l'Institut océanographique, 23-32.

Bundy JG, Davey MP, Viant MR (2008) Environmental metabolomics: a critical review and future perspectives. Metabolomics, 5, 3-21.

Burriesci MS, Raab TK, Pringle JR (2012) Evidence that glucose is the major transferred metabolite in dinoflagellate-cnidarian symbiosis. The Journal of Experimental Marine Biology and Ecology, 215, 3467-77.

Bythell JC, Douglas AE, Sharp VA, Searle JB, Brown BE (1997) Algal genotype and photoacclimatory responses of the symbiotic alga Symbiodiniumin natural populations of the sea anemone Anemonia viridis. Proceedings of the Royal Society B: Biological Sciences, 264, 1277-1282.

Carroll S, Blanquet R (1984) Alanine uptake by isolated zooxanthellae of the mangrove jellyfish, Cassiopea xamachana. I. Transport mechanisms and utilization. The Biological Bulletin, 166, 409-418.

Cates N, Mclaughlin J (1976) Differences of ammonia metabolism in symbiotic and aposymbiotic Condylactis and Cassiopea spp. Journal of Experimental Marine Biology and Ecology, 21, 1-5.

Catmull J, Yellowlees D, Miller D (1987) NADP-dependent glutamate dehydrogenase from Acropora formosa: purification and properties. Marine Biology, 95, 559-563. 
Clark KB, Jensen KR (1982) Effects of temperature on carbon fixation and carbon budget partitioning in the zooxanthellal symbiosis of Aiptasia pallida (Verrill). Journal of Experimental Marine Biology and Ecology, 64, 215-230.

Coffroth M, Santos S (2005) Genetic diversity of symbiotic dinoflagellates in the genus Symbiodinium. Protist, 156, 19-34.

Coles SL, Jokiel PL (1978) Synergistic effects of temperature, salinity and light on the hermatypic coral Montipora verrucosa. Marine Biology, 49, 187-195.

Cook C, Muller-Parker G, D'Elia C (1992) Ammonium enhancement of dark carbon fixation and nitrogen limitation in symbiotic zooxanthellae-effects of feeding and starvation of the sea anemone Aiptasia pallida. Limnology and Oceanography, 37, 131-139.

Cowman AF, Berry D, Baum J (2012) The cellular and molecular basis for malaria parasite invasion of the human red blood cell. The Journal of cell biology, 198, 96171.

Crossland C, Barnes D (1977) Nitrate assimilation enzymes from two hard corals, Acropora acuminata and Goniastrea australensis. Comparative Biochemistry and Physiology Part B, 57, 151-157.

D'Elia C (1977) Uptake and release of dissolved phosphorus by reef corals. Limnology and Oceanography, 22, 301-315.

D'Elia C, Domotor S, Webb K (1983) Nutrient uptake kinetics of freshly isolated zooxanthellae. Marine Biology, 75, 157-167.

Davies P (1991) Effect of daylight variations on the energy budgets of shallow-water corals. Marine Biology, 144, 137-144.

Davy SK, Allemand D, Weis VM (2012) Cell biology of cnidarian-dinoflagellate symbiosis. Microbiology and Molecular Biology Reviews, 76, 229-261.

Davy SK, Turner JR (2003) Early development and acquisition of Zooxanthellae in the temperate symbiotic sea anemone Anthopleura ballii (Cocks). The Biological bulletin, 205, 66-72.

Davy S, Turner J, Lucas I (1997) The nature of temperate anthozoan-dinoflagellate symbioses. In: The 8th International Coral Reef Symposium, pp. 1307-1312. ReefBase, Penang, Malaysia. 
Davy SK, Withers KJJT, Hinde R (2006) Effects of host nutritional status and seasonality on the nitrogen status of zooxanthellae in the temperate coral Plesiastrea versipora (Lamarck). Journal of Experimental Marine Biology and Ecology, 335, 256-265.

Deane EM, O'Brien RW (1981) Uptake of phosphate by symbiotic and free-living dinoflagellates. Archives of Microbiology, 128, 307-310.

Díaz-Almeyda E, Thomé PE, Hafidi M El, Iglesias-Prieto R (2011) Differential stability of photosynthetic membranes and fatty acid composition at elevated temperature in Symbiodinium. Coral Reefs, 30, 217-225.

Dimond JL, Bingham BL, Muller-Parker G, Wuesthoff K, Francis L (2011) Seasonal stability of a flexible algal-cnidarian symbiosis in a highly variable temperate environment. Limnology and Oceanography, 56, 2233-2242.

Dixon R (2001) Natural products and plant disease resistance. Nature, 411, 843-847.

Domotor SL, Elia CFD, Studies E (1984) Nutrient uptake kinetics and growth of zooxanthellae maintained in laboratory culture. Marine Biology, 80, 93-101.

Douglas AE (2010) The Symbiotic Habit. Princeton University Press.

Dudler N, Miller D (1988) Characterization of 2 glutamate-dehydrogenases from the symbiotic microalga Symbiodinium microadriaticum isolated from the coral Acropora formosa. Marine Biology, 97, 427-430.

Dykens JA, Shick JM, Benoit C, Buettner GR, Winston GW (1992) Oxygen radical production in the sea anemone Anthopleura elegantissima and its endosymbiotic algae. Journal of Experimental Biology, 168, 219-241.

Emerson R (1929) Chlorophyll Content and Rate of Photosynthesis. Proceedings of the National Academy of Sciences of the United States of America, 15, 281-4.

Fancy S, Rumpel K (2008) GC-MS-Based Metabolomics. In: Methods in Pharmacology and Toxicology: Biomarker Methods in Drug Discovery and Development, pp. 317340.

Ferrier-Pagès C, Allemand D, Gattuso J, Jaubert J, Rassoulzadegan R (1998) Microheterotrophy in the zooxanthellate coral Stylophora pistillata: effects of light and ciliate density. Limnology and Oceanography, 43, 1639-1648.

Fiehn O (2001) Combining genomics, metabolome analysis, and biochemical modelling to understand metabolic networks. Comparative and Functional Genomics, 2, 155- 
168.

Fiehn O, Kopka J, Dörmann P et al. (2000) Metabolite profiling for plant functional genomics. Nature biotechnology, 18, 1157-61.

Fitt WK, McFarland FK, Warner ME, Chilcoat GC (2000) Seasonal patterns of tissue biomass and densities of symbiotic dinoflagellates in reef corals and relation to coral bleaching. Limnology and Oceanography, 45, 677-685.

Fouad WMM (2004) Metabolic engineering for beta-alanine overproduction and stress tolerance in plants: expression of Escherichia coli L- aspartate-alpha-decarboxylase in transgenic tobacco.

Franklin DJ, Hoegh-Guldberg O, Jones RJ, Berges JA (2004) Cell death and degeneration in the symbiotic dinoflagellates of the coral Stylophora pistillata during bleaching. Marine Ecology Progress Series, 272, 117-130.

Funk CD (2001) Prostaglandins and leukotrienes: advances in eicosanoid biology. Science, 294, 1871-5.

Gabriel DG, Rodney VS (2006) Coral reef resilience and resistance to bleaching. IUCN, Gland, Switzerland.

Galang MGM, Macabeo APG, Chang W-C, Isobe M, Aguinaldo MAM (2016) Glucosides from the unripe fruit juice of Carica papaya Linn. (Caricaceae) cultivar "Red Lady" with antioxidant activity. Journal of Functional Foods, 22, 358-362.

Garrett TA, Schmeitzel JL, Klein JA, Hwang JJ, Schwarz JA (2013) Comparative lipid profiling of the cnidarian Aiptasia pallida and Its dinoflagellate symbiont. PloS one, 8, e57975.

Gibbons CL (2008) Carbon Flux in the Temperate Zooxanthellate Sea Anemone Anthpleura aureoradiata. Victoria University of Wellington.

Godinot C, Ferrier-Pagés C, Grover R (2009) Control of phosphate uptake by zooxanthellae and host cells in the scleractinian coral Stylophora pistillata. Limnology and Oceanography, 54, 1627-1633.

Gordon BR, Leggat W (2010) Symbiodinium-invertebrate symbioses and the role of metabolomics. Marine drugs, 8, 2546-68.

Gorr TA, Gassmann M, Wappner P (2006) Sensing and responding to hypoxia via HIF in model invertebrates. Journal of insect physiology, 52, 349-64. 
Grover R, Maguer J, Reynaud-Vaganay S, Ferrier-Pagès C (2002) Uptake of ammonium by the scleractinian coral Stylophora pistillata: effect of feeding, light, and ammonium concentrations. Limnology and Oceanography, 47, 782-790.

Guerzoni ME, Lanciotti R, Cocconcelli PS (2001) Alteration in cellular fatty acid composition as a response to salt, acid, oxidative and thermal stresses in. Cell, 22552264.

Gunnersen J, Yellowlees D, Miller DJ (1988) Marine Biology of Symbiodinium microadriaticum. Marine Biology, 596, 593-596.

Guy C, Kaplan F, Kopka J, Selbig J, Hincha DK (2008) Metabolomics of temperature stress. Physiologia plantarum, 132, 220-35.

Harding JJ, Hassett PC, Rixon KC, Bron AJ, Harvey DJ (1999) Sugars including erythronic and threonic acids in human aqueous humour. Current eye research, 19, $131-6$.

Harland A, Davies P (1995) Symbiont photosynthesis increases both respiration and photosynthesis in the symbiotic sea anemone Anemonia viridis. Marine Biology, 123, 715-722.

Hillyer KE, Tumanov S, Villas-Bôas S, Davy SK (2015) Metabolite profiling of symbiont and host during thermal stress and bleaching in a model cnidarian-dinoflagellate symbiosis. The Journal of experimental biology, jeb.128660-.

Hoegh-Guldberg O (1999) Climate change, coral bleaching and the future of the world's coral reefs. Marine and Freshwater Research, 50, 839-866.

Hoegh-Guldberg O, Mumby P, Hooten A (2007) Coral reefs under rapid climate change and ocean acidification. Science, 318, 1737-1742.

Hoegh-Guldberg O, Oritz JC, Dove S (2011) The future of coral reefs. Science, 334, 1494-1496.

von Holt C, von Holt M (1968) Transfer of photosynthetic products from zooxanthellae to coelenterate hosts. Comparative Biochemistry and Physiology, 24, 73-81.

Howe JN (2013) The Genetic and Physiological Characteristics of the Symbiodinium spp . in the endemic anemone Anthopleura aureoradiata.

Hughes TP, Baird AH, Bellwood DR et al. (2003) Climate change, human impacts, and the resilience of coral reefs. Science, 301, 929-933. 
Hulbert AJ (2003) Life, death and membrane bilayers. The Journal of experimental biology, 206, 2303-2311.

Hymes J, Wolf B (1996) Biotinidase and its roles in biotin metabolism. Clinica chimica acta; international journal of clinical chemistry, 255, 1-11.

Ianson D, Pond S, Parsons T (2001) The Spring Phytoplankton Bloom in the Coastal Temperate Ocean: Growth Criteria and Seeding from Shallow Embayments. Journal of Oceanography, 57, 723-734.

Iglesias-prieto R, Trench RK (1994) Acclimation and adaptation to irradiance in symbiotic dinoflagellates. 1.Responses of the photosynthetic unit to changes in photon flux density. Marine Ecology Progress Series, 113, 163-176.

Imbs AB, Yakovleva IM (2012) Dynamics of lipid and fatty acid composition of shallowwater corals under thermal stress: An experimental approach. Coral Reefs, 31, 4153.

Imbs AB, Yakovleva IM, Dautova TN, Bui LH, Jones P (2014) Diversity of fatty acid composition of symbiotic dinoflagellates in corals: Evidence for the transfer of host PUFAs to the symbionts. Phytochemistry, 101, 76-82.

Ishikura M, Adachi K, Maruyama T (1999) Zooxanthellae release glucose in the tissue of a giant clam, Tridacna crocea. Marine Biology, 133, 665-673.

Jackson A, Miller D, Yellowlees D (1989) Phosphorus Metabolism in the CoralZooxanthellae Symbiosis: Characterization and Possible Roles of Two Acid Phosphatases in the Algal Symbiont Symbiodinium. Proceedings of the Royal Society of London. Series B, Biological Sciences, 238, 193-202.

Jänne J, Alhonen L, Keinänen TA et al. (2005) Animal disease models generated by genetic engineering of polyamine metabolism. Journal of cellular and molecular medicine, 9, 865-82.

Jänne J, Alhonen L, Pietilä M, Keinänen TA (2004) Genetic approaches to the cellular functions of polyamines in mammals. European journal of biochemistry / FEBS, 271, 877-94.

Jiang PL, Pasaribu B, Chen CS (2014) Nitrogen-deprivation elevates lipid levels in Symbiodinium spp. by lipid droplet accumulation: Morphological and compositional analyses. PLoS ONE, 9, 1-10. 
Kaiser MJ (2011) Marine Ecology: Processes, Systems, and Impacts. OUP Oxford.

Kaplan F, Kopka J, Haskell DW et al. (2004) Exploring the Temperature-Stress Metabolome of Arabadopsis. Plant physiology, 136, 4159-4168.

Karasawa T, Hodge A, Fitter AH (2012) Growth, respiration and nutrient acquisition by the arbuscular mycorrhizal fungus Glomus mosseae and its host plant Plantago lanceolata in cooled soil. Plant, Cell and Environment, 35, 819-828.

Kazandjian A, Shepherd VA, Rodriguez-Lanetty M et al. (2008) Isolation of Symbiosomes and The Symbiosome Membrane Complex from The Zoanthid Zoanthus Robustus. Phycologia, 47, 294-306.

Ketudat Cairns JR, Mahong B, Baiya S, Jeon J-S (2015) $\beta$-Glucosidases: Multitasking, moonlighting or simply misunderstood? Plant science : an international journal of experimental plant biology, 241, 246-59.

Kim YS (2002) Malonate metabolism: biochemistry, molecular biology, physiology, and industrial application. Journal of biochemistry and molecular biology, 35, 443-451.

Kneeland J, Hughen K, Cervino J, Hauff B, Eglinton T (2013) Lipid biomarkers in Symbiodinium dinoflagellates: New indicators of thermal stress. Coral Reefs, 32, 923-934.

Kopp C, Pernice M, Domart-Coulon I (2013) Highly Dynamic Cellular-Level Response of Symbiotic Coral to a Sudden Increase in Environmental Nitrogen. mBio, 4, 1-9.

Krüger A, Grüning N-M, Wamelink MMC et al. (2011) The pentose phosphate pathway is a metabolic redox sensor and regulates transcription during the antioxidant response. Antioxidants \& redox signaling, 15, 311-324.

Van Kuilenburg ABP, Stroomer AEM, Van Lenthe H, Abeling NGGM, Van Gennip AH (2004) New insights in dihydropyrimidine dehydrogenase deficiency: a pivotal role for beta-aminoisobutyric acid? The Biochemical journal, 379, 119-124.

Kültz D (2005) Molecular and Evolutionary Basis of the Cellular Stress Response. Annual Review of Physiology, 67, 225-257.

LaJeunesse $\mathrm{T}$ (2001) Investigating the biodiversity, ecology, and phylogeny of endosymbiotic dinoflagellates in the genus Symbiodinium using the ITS region: in search of a "species" level. Journal of Phycology, 880, 866-880.

LaJeunesse TC (2002) Diversity and community structure of symbiotic dinoflagellates 
from Caribbean coral reefs. Marine Biology, 141, 387-400.

Lajeunesse TC (2005) "Species" radiations of symbiotic dinoflagellates in the Atlantic and Indo-Pacific since the Miocene-Pliocene transition. Molecular biology and evolution, 22, 570-81.

LaJeunesse TC, Pettay DT, Sampayo EM et al. (2010) Long-standing environmental conditions, geographic isolation and host-symbiont specificity influence the relative ecological dominance and genetic diversification of coral endosymbionts in the genus Symbiodinium. Journal of Biogeography, 37, 785-800.

LaJeunesse TC, Smith RT, Finney J, Oxenford H (2009) Outbreak and persistence of opportunistic symbiotic dinoflagellates during the 2005 Caribbean mass coral "bleaching" event. Proceedings of the Royal Society B: Biological Sciences, 276, 4139-48.

Lajeunesse TC, Trench RK (2000) Biogeography of two species of Symbiodinium (Freudenthal) inhabiting the intertidal sea anemone Anthopleura elegantissima (Brandt). Biological Bulletin, 199, 126-134.

Lankadurai B, Ngato E, Simpson M (2013) Environmental metabolomics: an emerging approach to study organism responses to environmental stressors. Environmental Reviews, 21, 180-205.

Leggat W, Hoegh-Guldberg O, Dove S, Yellowlees D (2007) Analysis of an EST library from the dinoflagellate ( Symbiodinium sp.) symbiont of reef-building corals 1 . Journal of Phycology, 43, 1010-1021.

Leggat W, Seneca F, Wasmund K et al. (2011) Differential responses of the coral host and their algal symbiont to thermal stress. PLOS ONE, 6, 1-11.

Leigh J, Hodge A, Fitter AH (2009) Arbuscular mycorrhizal fungi can transfer substantial amounts of nitrogen to their host plant from organic material. The New phytologist, 181, 199-207.

Leonardi R, Zhang Y-M, Rock CO, Jackowski S (2005) Coenzyme A: back in action. Progress in lipid research, 44, 125-53.

Lesser MP (1996) Elevated temperatures and ultraviolet radiation cause oxidative stress and inhibit photosynthesis in symbiotic dinoflagellates. Limnology and Oceanography, 41, 271-283. 
Lesser MP (2006) Oxidative Stress in Marine Environments: Biochemistry and Physiological Ecology. Annual Review of Physiology, 68, 253-278.

Lesser MP, Farrell JH (2004) Exposure to solar radiation increases damage to both host tissues and algal symbionts of corals during thermal stress. Coral Reefs, 23, 367377.

Levinton JS (1995) Critical Factors in Plankton abundance. In: Marine Biology: Function, Biodiversity, Ecology, pp. 173-193. Oxford University Press.

Levy O, Achituv Y, Yacobi YZ, Stambler N, Dubinsky Z (2006) The impact of spectral composition and light periodicity on the activity of two antioxidant enzymes (SOD and CAT) in the coral Favia favus. Journal of Experimental Marine Biology and Ecology, 328, 35-46.

Lewis DH, Smith DC (1971) The Autotrophic Nutrition of Symbiotic Marine Coelenterates with Special Reference to Hermatypic Corals. I. Movement of Photosynthetic Products between the Symbionts. Proceedings of the Royal Society B: Biological Sciences, 178, 111-129.

Lipschultz F, Cook C (2002) Uptake and assimilation of $15 \mathrm{~N}$-ammonium by the symbiotic sea anemones Bartholomea annulata and Aiptasia pallida: conservation versus recycling of nitrogen. Marine Biology, 140, 489-502.

Lisec J, Schauer N, Kopka J, Willmitzer L, Fernie AR (2006) Gas chromatography mass spectrometry-based metabolite profiling in plants. Nature protocols, 1, 387-96.

Liu G, Xiao L, Cao W et al. (2016) Changes in the metabolome of rats after exposure to arginine and $\mathrm{N}$-carbamylglutamate in combination with diquat, a compound that causes oxidative stress, assessed by ${ }^{1}$ H NMR spectroscopy. Food Funct., 964-974.

Logan DDK, LaFlamme AC, Weis VM, Davy SK (2010) Flow-Cytometric Characterization of the Cell-Surface Glycans of Symbiotic Dinoflagellates (Symbiodinium Spp.)1. Journal of Phycology, 46, 525-533.

Loh WKW, Loi T, Carter D, Hoegh-Guldberg O (2001) Genetic variability of the symbiotic dinoflagellates from the wide ranging coral species Seriatopora hystrix and Acropora longicyathus in the Indo-West Pacific. Marine Ecology Progress Series, 222, 97-107.

Loram JE, Trapido-Rosenthal HG, Douglas AE (2007) Functional significance of 
genetically different symbiotic algae Symbiodinium in a coral reef symbiosis. Molecular ecology, 16, 4849-57.

Markell D, Trench R (1993) Macromolecules exuded by symbiotic dinoflagellates in culture: Amino ascid and sugar composition. Journal of phycology, 12, 19-31.

Markell DA, Wood-Charlson EM (2010) Immunocytochemical evidence that symbiotic algae secrete potential recognition signal molecules in hospite. Marine Biology, 157, $1105-1111$.

Mayfield AB, Gates RD (2007) Osmoregulation in anthozoan-dinoflagellate symbiosis. Comparative Biochemistry and Physiology - A Molecular and Integrative Physiology, 147, 1-10.

McAuley PJ, Cook CB (1994) Effects of host feeding and dissolved ammonium on cell division and nitrogen status of zooxanthellae in the hydroid Myrionema amboinense. Marine Biology, 121, 343-348.

Miller D, Yellowlees D (1989) Inorganic nitrogen uptake by symbiotic marine cnidarians-a critical review. Proc. R. Soc. Lond.B. Proceedings of the Royal Society B: Biological Sciences, 237, 109-125.

Moncada S, Vane JR (1978) Pharmacology and endogenous roles of prostaglandin endoperoxides, thromboxane A2, and prostacyclin. Pharmacological reviews, 30, 293-331.

Morar SR, Bury SJ, Wilkinson SP, Davy SK (2011) Sedimentary nitrogen uptake and assimilation in the temperate zooxanthellate sea anemone Anthopleura aureoradiata. Journal of Experimental Marine Biology and Ecology, 399, 110-119.

Motti C (2012) Environmental marine metabolomics: from whole organism system biology to ecosystem management. Journal of Marine Science Research and Development, 2, 2-3.

Muller-Parker G, Cook C, D'Elia C (1990) Feeding affects phosphate fluxes in the symbiotic sea anemone Aiptasia pallida*. Marine Ecology Progress Series, 60, $283-$ 290.

Muller-Parker G, Davy S (2001) Temperate and tropical algal-sea anemone symbioses. Invertebrate Biology, 120, 104-123.

Muller-Parker G, McCloskey LR, Hoegh-Guldberg O, McAuley PJ (1996) Effect of 
Ammonium Enrichment on Animal and Algal Biomass of. Pacific Science, 48, 273283.

Muscatine L (1967) Glycerol excretion by symbiotic algae from corals and Tridacna and its control by the host. Science, 156, 2-5.

Muscatine L (1990) The role of symbiotic algae in carbon and energy flux in reef corals. In: Coral reefs (ed Dubinsky Z), pp. 75-87. Elsevier, Amsterdam, The Netherlands.

Muscatine L, D'Elia C (1978) Uptake, retention, and release of ammonium by reef corals. Limnology and Oceanography, 23, 725-734.

Muscatine L, Falkowski PG, Dubinsky Z, Cook PA, McCloskey LR (1989) The Effect of External Nutrient Resources on the Population Dynamics of Zooxanthellae in a Reef Coral. Proceedings of the Royal Society B: Biological Sciences, 236, 311-324.

Muscatine L, Falkowski PG, Porter JW, Dubinsky Z (1984) Fate of photosynthetic fixed carbon in light- and shade-adapted colonies of the symbiotic coral Stylophora pistillata. Proceedings of the Royal Society B: Biological Sciences, 222, 181-202.

Muscatine L, Ferrier-Pagès C, Blackburn A et al. (1998) Cell-specific density of symbiotic dinoflagellates in tropical anthozoans. Coral Reefs, 17, 329-337.

Muscatine L, Hand C (1958) Direct evidence for the transfer of materials from symbiotic algae to the tissues of a coelenterate. Proceedings of the National Academy of Sciences of the United States of America, 44, 1259-1263.

Muscatine L., Steen G (1987) Low Temperature Evokes Rapid Exocytosis of Symbiotic Algae by a Sea Anemone. Biological Bulletin, 172, 246-263.

Nakamura H, Kawase Y, Maruyama K, Murai A (1998) Studies on Polyketide Metabolites of a Symbiotic Dinoflagellate, Symbiodinium sp.: A New C30 Marine Alkaloid, Zooxanthellamine, a Plausible Precursor for Zoanthid Alkaloids. Bulletin of the Chemical Society of Japan, 71, 781-787.

Needleman P, Turk J, Jakschik BA, Morrison AR, Lefkowith JB (1987) Arachidonic acid metabolism. Preventive medicine, 16, 503-509.

Nii CM, Muscatine L (1997) Oxidative stress in the symbiotic sea anemone Aiptasia pulchella (Carlgren, 1943): Contribution of the animal to superoxide ion production at elevated temperature. Biological Bulletin, 192, 444-456.

Nordstokke DW, Zumbo BD (2010) A new nonparametric levene test for equal variances. 
Psicologica, 31, 401-403.

Nordstokke DW, Zumbo BD, Cairns SL, Saklofske DH (2011) The operating characteristics of the nonparametric Levene test for equal variances with assessment and evaluation data. Practical Assessment, Research \& Evaluation, 16, 1-8.

Odum H, Odum E (1955) Trophic structure and productivity of a windward coral reef community on Eniwetok Atol. Ecological monographs, 25, 291-320.

Oliver SG, Winson MK, Kell DB, Baganz F (1998) Systematic functional analysis of the yeast genome. Trends in biotechnology, 16, 373-378.

Pacheco-Alvarez D, Solórzano-Vargas RS, Del Río AL (2002) Biotin in metabolism and its relationship to human disease. Archives of medical research, 33, 439-47.

Papina M, Meziane T, van Woesik R (2003) Symbiotic zooxanthellae provide the hostcoral Montipora digitata with polyunsaturated fatty acids. Comparative biochemistry and physiology. Part B, Biochemistry \& molecular biology, 135, 533-537.

Papina M, Meziane T, van Woesik R (2007) Acclimation effect on fatty acids of the coral Montipora digitata and its symbiotic algae. Comparative Biochemistry and Physiology - B Biochemistry and Molecular Biology, 147, 583-589.

Pochon X, LaJeunesse TC, Pawlowski J (2004) Biogeographic partitioning and host specialization among foraminiferan dinoflagellate symbionts (Symbiodinium; Dinophyta). Marine Biology, 146, 17-27.

Pollitt RJ, Green A, Smith R (1985) Excessive excretion of $\beta$-alanine and of 3hydroxypropionic, R- and S-3-aminoisobutyric, R- and S-3-hydroxyisobutyric and S-2-(hydroxymethyl)butyric acids probably due to a defect in the metabolism of the corresponding malonic semialdehydes. Journal of Inherited Metabolic Disease, 8, $75-79$.

Pomeroy L, Kuenzler E (1969) Phosphorus turnover by coral reef animals. In: Symposium on radioecology. National Technical Information Service (eds Nelson D, Evans F), pp. 474-482. Springfield, VA.

Pontasch S (2014) Living on the Edge: Protective Mechanisms Underlying Thermal Tolerance in High Latitude Symbiodinium spp . Victoria University of Wellington.

Rahav O, Dubinsky Z, Achituv Y, Falkowski P (1989) Ammonium metabolism in the zooxanthellate coral, Stylophora pistillata. Proceedings of the Royal Society B: 
Biological Sciences, 236, 325-337.

Rasmussen S, Parsons AJ, Fraser K, Xue H, Newman JA (2008) Metabolic profiles of Lolium perenne are differentially affected by nitrogen supply, carbohydrate content, and fungal endophyte infection. Plant physiology, 146, 1440-1453.

Richier S, Furla P, Plantivaux A, Merle P-L, Allemand D (2005) Symbiosis-induced adaptation to oxidative stress. The Journal of experimental biology, 208, 277-285.

Roberts JM, Davies PS, Fixter LM (1999) Symbiotic anemones can grow when starved: nitrogen budget for Anemonia viridis in ammonium-supplemented seawater. Marine Biology, 133, 29-35.

Roberts J, Fixter L, Davies P (2001) Ammonium metabolism in the symbiotic sea anemone Anemonia viridis. Hydrobiologia, 461, 25-35.

Rodriguez-Lanetty M, Krupp DA, Weis VM (2004) Distinct ITS types of Symbiodinium in Clade $\mathrm{C}$ correlate with cnidarian/dinoflagellate specificity during onset of symbiosis. Marine Ecology Progress Series, 275, 97-102.

Roepke J, Bozzo GG (2015) Arabidopsis thaliana $\beta$-glucosidase BGLU15 attacks flavonol 3-O- $\beta$-glucoside-7-O- $\alpha$-rhamnosides. Phytochemistry, 109, 14-24.

Roessner U, Wagner C, Kopka J, Trethewey RN, Willmitzer L (2000) Technical advance: simultaneous analysis of metabolites in potato tuber by gas chromatography-mass spectrometry. The Plant journal : for cell and molecular biology, 23, 131-42.

Rosen BR (1988) Progress, problems and patterns in the biogeography of reef corals and other tropical marine organisms. Helgoländer Meeresuntersuchungen, 42, 269-301.

Rosic NN, Pernice M, Dove S, Dunn S, Hoegh-Guldberg O (2011) Gene expression profiles of cytosolic heat shock proteins Hsp70 and Hsp90 from symbiotic dinoflagellates in response to thermal stress: Possible implications for coral bleaching. Cell Stress and Chaperones, 16, 69-80.

Rouphael Y, Franken P, Schneider C et al. (2015) Arbuscular mycorrhizal fungi act as biostimulants in horticultural crops. Scientia Horticulturae, 196, 91-108.

Rowan R (1998) Review - Diversity and Ecology of Zooxanthellae on Coral Reefs. Journal of Phycology, 34, 407-417.

Rowan R, Knowlton N (1995) Intraspecific diversity and ecological zonation in coralalgal symbiosis. Proceedings of the National Academy of Sciences of the United 
States of America, 92, 2850-2853.

Rowan R, Powers DA (1991) A molecular genetic classification of zooxanthellae and the evolution of animal-algal symbioses. Science (New York, N.Y.), 251, 1348-51.

Rowan R, Powers D a (1992) Ribosomal RNA sequences and the diversity of symbiotic dinoflagellates (zooxanthellae). Proceedings of the National Academy of Sciences of the United States of America, 89, 3639-3643.

Saito K, Matsuda F (2010) Metabolomics for functional genomics, systems biology, and biotechnology. Annual review of plant biology, 61, 463-89.

Sampayo EM, Franceschinis L, Hoegh-Guldberg O, Dove S (2007) Niche partitioning of closely related symbiotic dinoflagellates. Molecular Ecology, 16, 3721-3733.

Samuelsson B (1991) Arachidonic acid metabolism: role in inflammation. Zeitschrift fur Rheumatologie, 50, 3-6.

Samuelsson B, Dahlén SE, Lindgren JA, Rouzer CA, Serhan CN (1987) Leukotrienes and lipoxins: structures, biosynthesis, and biological effects. Science (New York, N.Y.), 237, 1171-1176.

Saxby T, Dennison WC, Hoegh-Guldberg O (2003) Photosynthetic responses of the coral Montipora digitata to cold temperature stress. Marine Ecology Progress Series, 248, $85-97$.

Schliemann W, Ammer C, Strack D (2008) Metabolite profiling of mycorrhizal roots of Medicago truncatula. Phytochemistry, 69, 112-46.

Schoenberg DA, Trench RK (1980) Genetic Variation in Symbiodinium (=Gymnodinium) microadriaticum Freudenthal, and Specificity in its Symbiosis with Marine Invertebrates. I. Isoenzyme and Soluble Protein Patterns of Axenic Cultures of Symbiodinium microadriaticum. Proceedings of the Royal Society B: Biological Sciences, 207, 405-427.

Smirnoff N, Cumbes QJ (1989) Hydroxyl radical scavenging activity of compatible solutes. Phytochemistry, 28, 1057-1060.

Smith SE, Smith FA (2011) Roles of arbuscular mycorrhizas in plant nutrition and growth: new paradigms from cellular to ecosystem scales. Annual review of plant biology, 62, 227-50.

Stanley Jr GD, van de Schootbrugge B (2009) The Evolution of the Coral - Algal 
Symbiosis. In: Coral Bleaching (eds van Oppen MJ., Lough JM), pp. 7-20. Springer.

Stat M, Carter D, Hoeghguldberg O (2006) The evolutionary history of Symbiodinium and scleractinian hosts-Symbiosis, diversity, and the effect of climate change. Perspectives in Plant Ecology, Evolution and Systematics, 8, 23-43.

Steele R, Goreau N (1977) The breakdown of symbiotic zooxanthellae in the sea anemone Phyllactis (Oulactis) flosculifera (Actinaria). Journal of zoology, 181, 421-437.

Steen RG, Angeles L (1987) Evidence for facultative heterotrophy in cultured zooxanthellae. Marine Biology, 95, 15-23.

Streamer M (1980) Urea and arginine metabolism in the hard coral, Acropora acuminata. Comparative Biochemistry and Physiology Part B: Comparative Biochemistry, 65, 669-674.

Stubbs CD, Smith AD (1984) The modification of mammalian membrane polyunsaturated fatty acid composition in relation to membrane fluidity and function. Biochimica et Biophysica Acta (BBA) - Reviews on Biomembranes, 779, 89-137.

Sulmon C, Van Baaren J, Cabello-Hurtado F et al. (2015) Abiotic stressors and stress responses: What commonalities appear between species across biological organization levels? Environmental Pollution, 202, 66-77.

Summons R, Boag T, Osmond C (1986) The effect of ammonium on photosynthesis and the pathway of ammonium assimilation in Gymnodinium microadriaticum in vitro and in symbiosis with tridacnid clams and corals. Proceedings of the Royal Society B: Biological Sciences, 227, 147-159.

Sunday JM, Bates AE, Kearney MR et al. (2014) Thermal-safety margins and the necessity of thermoregulatory behavior across latitude and elevation. Proceedings of the National Academy of Sciences of the United States of America, 111, 5610-5.

Sutton D, Hoegh-Guldberg O (1990) Host-zooxanthella interactions in four temperate marine invertebrate symbioses: assessment of effect of host extracts on symbionts. The Biological Bulletin, 178, 175-186.

Tchernov D, Gorbunov MY, de Vargas C et al. (2004) Membrane lipids of symbiotic algae are diagnostic of sensitivity to thermal bleaching in corals. Proceedings of the National Academy of Sciences of the United States of America, 101, 13531-5.

Thornhill DJ, Kemp DW, Bruns BU, Fitt WK, Schmidt GW (2008) Correspondence 
between cold tolerance and temperate biogeography in a western Atlantic Symbiodinium (Dinophyta) lineage. Journal of Phycology, 44, 1126-1135.

Tracy R (1976) A Model of the Dynamic Exchanges of Water and Energy between a Terrestrial Amphibian and Its Environment. Ecological Monographs, 46, 293-326.

Trench R (1971a) Physiology and biochemistry of zooxanthellae symbiotic with marine coelenterates. III. Effect of homogenates of host tissues on excretion of photosynthetic products in vitro by zooxanthellae from two marine coelenterates. Proceedings of the Royal Society B: Biological Sciences, 177, 251-264.

Trench R (1971b) Physiology and biochemistry of zooxanthellae symbiotic with marine coelenterates. II. Liberation of fixed C14 by zooxanthellae in vitro. Proceedings of the Royal Society B: Biological Sciences, 177, 237-250.

Trench R (1993) Microalgal-Invertebrate Symbioses - A Review. Endocytobiosis and Cell Research, 9, 135 - 175.

Valiela I (1995) Marine Ecological Processes. Springer Science \& Business Media.

Vandermeulen JH, Davis ND, Muscatine L (1972) The effect of inhibitors of photosynthesis on zooxanthellae in corals and other marine invertebrates. Marine Biology, 16, 185-191.

Venn AA, Loram JE, Douglas AE (2008) Photosynthetic symbioses in animals. Journal of Experimental Botany, 59, 1069-1080.

Venn A, Tambutté E, Lotto $\mathrm{S}$ et al. (2009) Imaging intracellular $\mathrm{pH}$ in a reef coral and symbiotic anemone. Proceedings of the National Academy of Sciences of the United States of America, 106, 16574-16579.

Veron JEN, Hoegh-Guldberg O, Lenton TM et al. (2009) The coral reef crisis: the critical importance of (less than) 350 ppm CO2. Marine Pollution Bulletin, 58, 1428-1436.

Vierheilig H, Schweiger P, Brundrett M (2005) An overview of methods for the detection and observation of arbuscular mycorrhizal fungi in roots. Physiologia Plantarum, 125, 393-404.

Villas-Bôas SG, Noel S, Lane GA, Attwood G, Cookson A (2006) Extracellular metabolomics: A metabolic footprinting approach to assess fiber degradation in complex media. Analytical Biochemistry, 349, 297-305.

Villas-bôas SG, Smart KF, Sivakumaran S, Lane GA (2011) Alkylation or Silylation for 
Analysis of Amino and Non-Amino Organic Acids by GC-MS? Metabolites, 3-20.

Visram S, Wiedenmann J, Douglas AE (2006) Molecular diversity of symbiotic algae of the genus Symbiodinium (Zooxanthellae) in cnidarians of the Mediterranean Sea. Journal of the Marine Biological Association of the UK, 86, 1281.

Vonholdt BM, Pollinger JP, Lohmueller KE et al. (2010) Genome-wide SNP and haplotype analyses reveal a rich history underlying dog domestication. Nature, $\mathbf{4 6 4}$, 898-902.

Wahid A, Gelani S, Ashraf M, Foolad MR (2007) Heat tolerance in plants: An overview. Environmental and Experimental Botany, 61, 199-223.

Wakefiel TS, Kempf SC (2001) Development of host- and symbiont-specific monoclonal antibodies and confirmation of the origin of the symbiosome membrane in a cnidarian-dinoflagellate symbiosis. The Biological bulletin, 200, 127-43.

Wang J, Douglas AE (1997) Nutrients, signals, and photosynthate release by symbiotic algae: the lmpact of taurine on the dinoflagellate alga Symbiodinium from the sea anemone Aiptasia pulchella. Plant physiology, 631-636.

Wang J, Douglas A (1998) Nitrogen recycling or nitrogen conservation in an algainvertebrate symbiosis? The Journal of experimental biology, 201, 2445-2453.

Wang JT, Douglas AE (1999) Essential amino acid synthesis and nitrogen recycling in an alga-invertebrate symbiosis. Marine Biology, 135, 219-222.

Warner ME, Fitt WK, Schmidt GW (1999) Damage to photosystem II in symbiotic dinoflagellates: a determinant of coral bleaching. Proceedings of the National Academy of Sciences of the United States of America, 96, 8007-12.

Weis VM (2008) Cellular mechanisms of Cnidarian bleaching: stress causes the collapse of symbiosis. The Journal of experimental biology, 211, 3059-3066.

Whitehead L, Douglas A (2003) Metabolite comparisons and the identity of nutrients translocated from symbiotic algae to an animal host. Journal of Experimental Biology, 206, 3149-3157.

Wilkerson F, Muscatine L (1984) Uptake and assimilation of dissolved inorganic nitrogen by a symbiotic sea anemone. Proceedings of the Royal Society B: Biological Sciences, 221, 71-86. 